I N T ER N ATIONAL MONETARY FUND

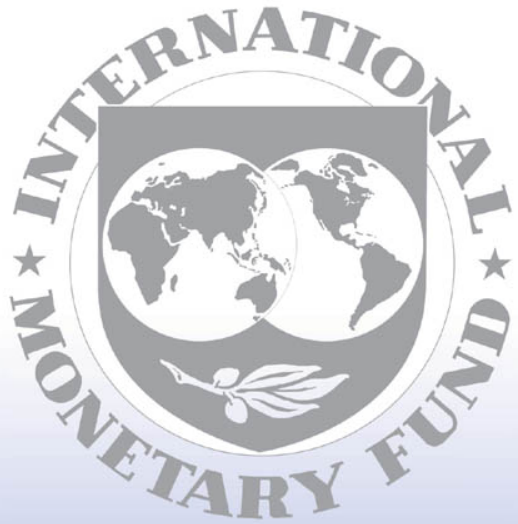

Staff

Country

Reports 


\section{Zambia: 2007 Article IV Consultation-Staff Report; Staff Statement; Public Information Notice on the Executive Board Discussion; and Statement by the Executive Director for Zambia}

Under Article IV of the IMF's Articles of Agreement, the IMF holds bilateral discussions with members, usually every year. In the context of the 2007 Article IV consultation with Zambia, the following documents have been released and are included in this package:

- $\quad$ the staff report for the 2007 Article IV consultation, prepared by a staff team of the IMF, following discussions that ended on September 26, 2007, with the officials of Zambia on economic developments and policies. Based on information available at the time of these discussions, the staff report was completed on November 20, 2007. The views expressed in the staff report are those of the staff team and do not necessarily reflect the views of the Executive Board of the IMF.

- $\quad$ a staff statement of December 7, 2007 updating information on recent developments.

- $\quad$ a Public Information Notice (PIN) summarizing the views of the Executive Board as expressed during its December 7, 2007 discussion of the staff report that concluded the Article IV consultation.

- $\quad$ a statement by the Executive Director for Zambia.

The document(s) listed below have been or will be separately released.

Selected Issues Paper

Statistical Appendix

The policy of publication of staff reports and other documents allows for the deletion of market-sensitive information.

To assist the IMF in evaluating the publication policy, reader comments are invited and may be sent by e-mail to publicationpolicy@imf.org.

Copies of this report are available to the public from

International Monetary Fund $\bullet$ Publication Services

$70019^{\text {th }}$ Street, N.W. • Washington, D.C. 20431

Telephone: (202) 623-7430 • Telefax: (202) 623-7201

E-mail: publications@imf.org Internet: http://www.imf.org

Price: $\$ 18.00$ a copy

\section{International Monetary Fund \\ Washington, D.C.}


This page intentionally left blank

CInternational Monetary Fund. Not for Redistribution 


\section{INTERNATIONAL MONETARY FUND}

ZAMBIA

Staff Report for the 2007 Article IV Consultation

Prepared by the Staff Representatives for the 2007 Consultation with Zambia

Approved by Sharmini Coorey and G. Russell Kincaid

November 20, 2007

Fund Relations. The three-year PRGF arrangement that expired on September 30, 2007 has been fully disbursed (SDR 220.1 million; 45 percent of quota). The Zambian government has requested continued support from the IMF in implementing its economic program under a lowaccess PRGF arrangement. Discussions on a new Fund-supported program are ongoing.

Staff Team. Messrs. Caramazza (head), Akatu, Mikkelsen (all AFR); Mr. Goldsworthy (FAD); Ms. Kufa (STA); and Ms. Mongrut (PDR). The team was assisted by Mr. Arnason (resident representative).

Mission Dates. September 12-26, 2007.

Interlocutors. Minister of Finance and National Planning Mr. Magande; Governor of the Bank of Zambia Mr. Fundanga; other senior officials; and representatives of civil society, donors, trade unions, and the business community. 


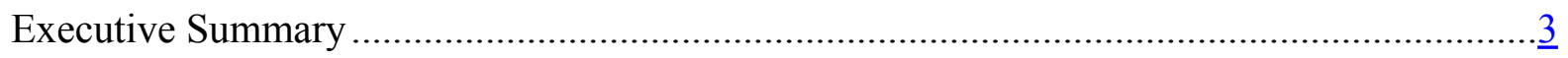

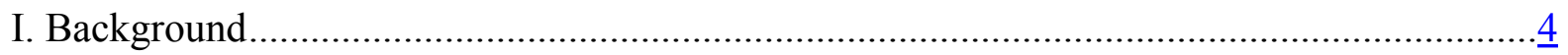

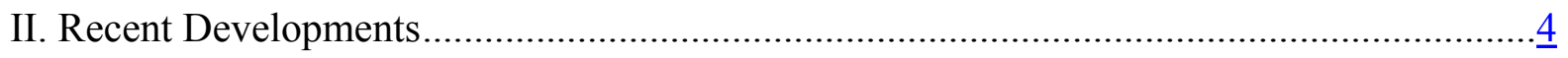

III. Policy Discussions ............................................................................................. $\frac{8}{8}$

A. Near-Term Policy Challenges and Medium-Term Outlook ................................ $\underline{8}$

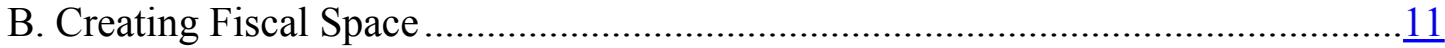

C. Public Financial Management and Budget Execution .......................................12

D. Managing Foreign Currency Inflows ...........................................................

E. Improving Conditions for Private Sector Growth .........................................14

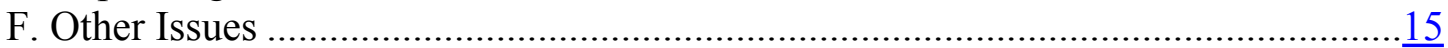

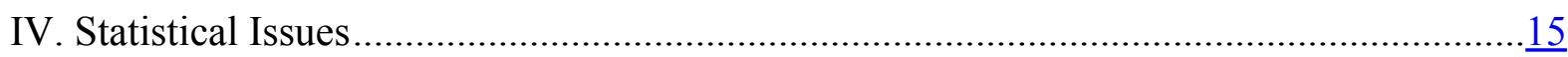

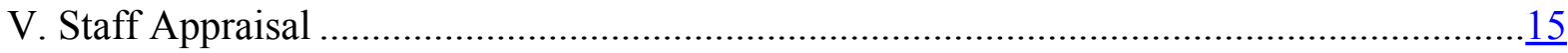

Tables

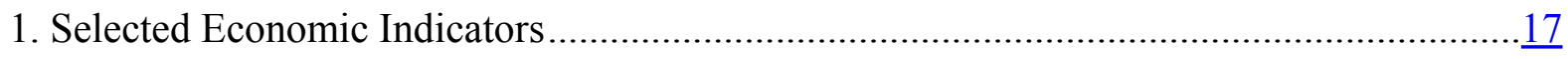

2. Fiscal Operations of the Central Government …................................................... 18

3. Fiscal Operations of the Central Government ......................................................... 19

4. Monetary Accounts ................................................................................................. $\frac{20}{21}$

5. Balance of Payments ............................................................................................ $\frac{21}{22}$

6. Financial Soundness Indicators, 2002-07............................................................ $\frac{22}{23}$

7. Millennium Development Goals ........................................................................... $\frac{23}{24}$

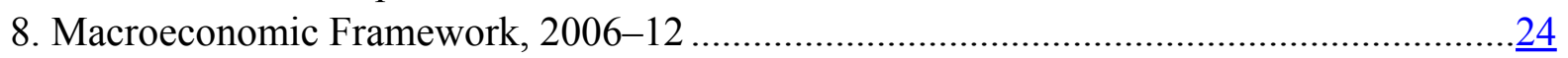

Box

1. Assessment of External Stability .......................................................................... 9

Annexes

I. Joint IMF/World Bank Debt Sustainability Analysis...............................................25

II. Zambia: Relations with the Fund ....................................................................... 37

III. Zambia: Relations with the World Bank Group ........................................................ 42

IV. Zambia: Statistical Issues .......................................................................... 


\section{EXECUTIVE SUMMARY}

The Zambian economy has performed well in recent years. This reflects strengthened macroeconomic policies, a favorable external environment, and extensive debt relief. Growth picked up further in 2006 and inflation has been held in check. The revival of the mining sector and high copper prices have contributed to a marked improvement in the trade balance and a steep appreciation of the currency.

The exchange rate and domestic economic and financial policies are consistent with external stability. The exchange rate is currently aligned with economic fundamentals, including the large improvement in the terms of trade.

Despite the favorable economic performance, budget execution and liquidity management have been problematic. Poor execution of the 2007 budget risks an excess of liquidity late in the year. That would complicate the conduct of monetary policy, which needs to hold firm to avoid an upsurge in inflation. The recent lowering of the statutory reserve requirements could further increase short-term pressure on monetary policy. The authorities believe that some of the budgetary releases already made will remain unspent, as it will not be possible to implement all of the planned projects. Furthermore, to ease the burden on monetary policy, releases for the last part of the year will be kept below planned levels in nonpriority areas. The authorities stressed that they were prepared to take whatever action needed to achieve the objectives of the monetary program.

To foster economic diversification, further raise growth, and enhance income opportunities for the poor, Zambia will need to address several medium-term policy challenges. These include creating fiscal space for increased spending on infrastructure and the social sectors; strengthening the capacity to undertake an expansion in public investments; managing the macroeconomic effects of high foreign exchange inflows; and further improving the conditions for private sector growth.

Addressing these challenges will require better budget planning and execution, closer coordination between fiscal and monetary policy, and enhancing the competitiveness of the non-mining export and import-competing sectors. A secure and efficient supply of energy will be critical to ensure that economic growth is not stifled by energy shortages and high costs. 


\section{BACKGROUND}

1. The Zambian economy has performed well in recent years because of stronger macroeconomic policies, a favorable external environment, and extensive debt relief. The broad-based economic expansion has benefited greatly from the revival of the mining sector and high copper prices. Public finances have improved markedly and inflation has been brought under control. The external position has strengthened significantly, mainly due to booming copper export receipts and debt relief through the HIPC Initiative and MDRI, which have contributed to a substantial real appreciation of the exchange rate and a buildup in international reserves.

2. The improved economic position presents a historic opportunity for Zambia to accelerate growth and reduce poverty. By maintaining macroeconomic stability and implementing the economic reforms in the Fifth National Development Plan (FNDP)Zambia's Poverty Reduction Strategy - economic growth could be sustained at a higher rate that would benefit the poor. ${ }^{1}$

\section{RECENT DEVELOPMENTS}

3. The positive economic performance of recent years continued in 2006 and the first half of 2007. Poor execution of the 2007 budget, however, has created considerable uncertainty about the end-year fiscal and monetary outcome.

- The economy continued to grow at a robust pace with inflation held in check (Table 1 and Figure 1). Real GDP accelerated to 6.2 percent in 2006 , driven by a vigorous expansion in mining, construction and telecommunications. Favorable terms of trade boosted real gross domestic income well above real GDP. Inflation, after falling to single digits in 2006,
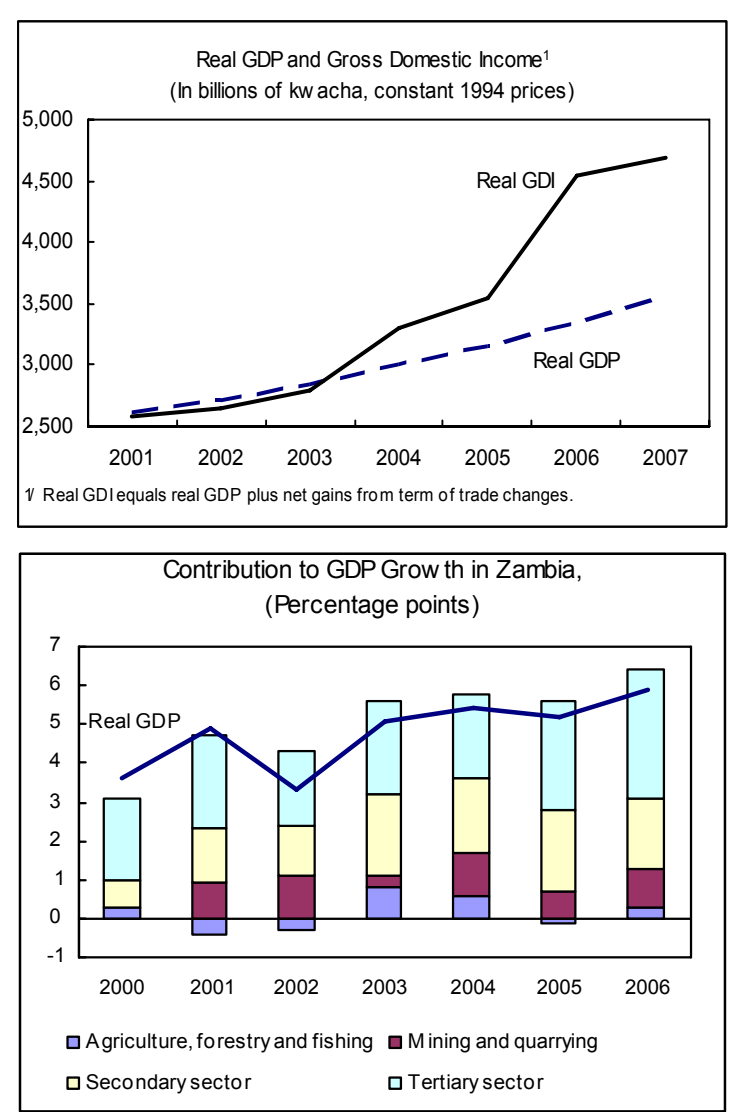

\footnotetext{
${ }^{1}$ See Zambia-Poverty Reducing Strategy (IMF Country Report No. 07/276) and the associated Joint Staff Advisory Note (IMF Country Report No. 07/277).
} 
Figure 1. Zambia: Selected Macroeconomic Indicators

Real GDP Grow th, 2000-07

(Annual percent change)

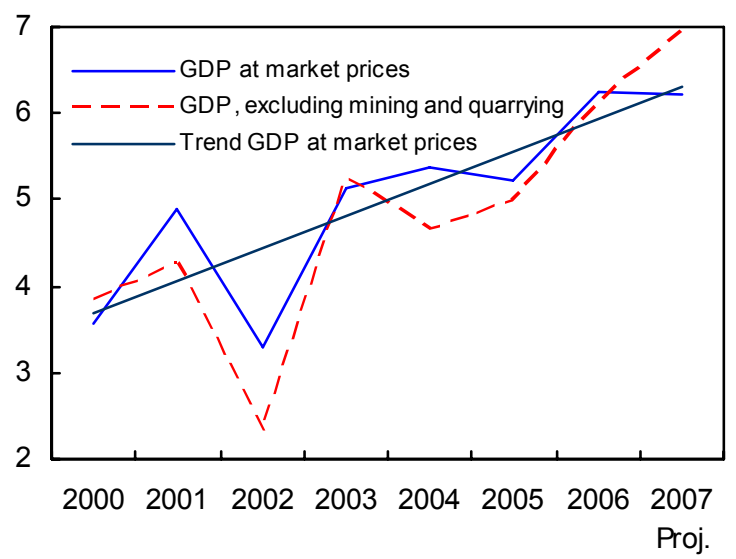

Overall Fiscal Balance and Financing, 2000-07

(Percent of GDP, excluding MDRl assistance)

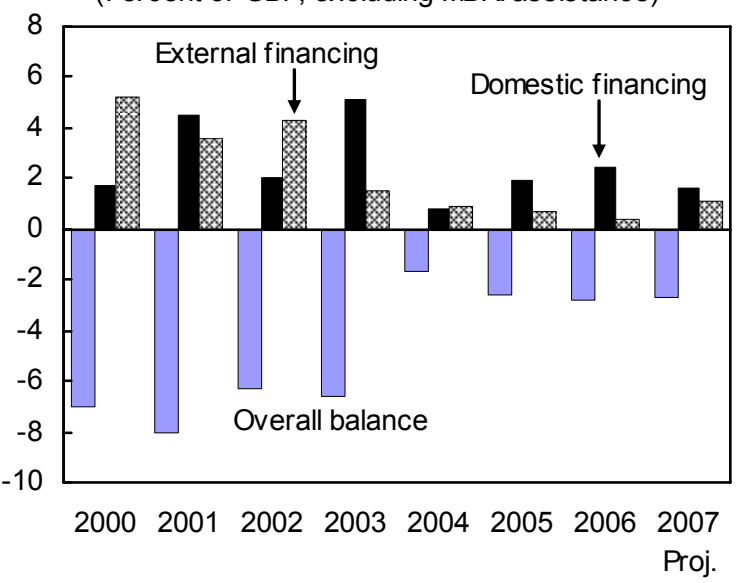

Current Account Balance, 2000-07

(Percent of GDP)

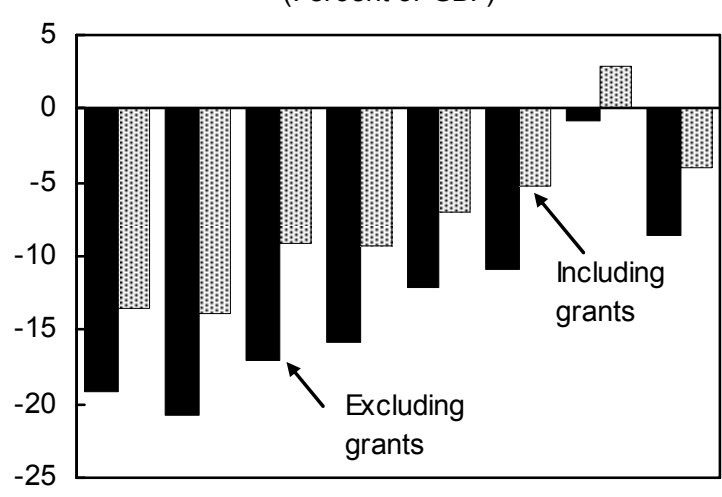

20002001200220032004200520062007

Proj.
Inflation, January 2000-October 2007

(12-month percent change)

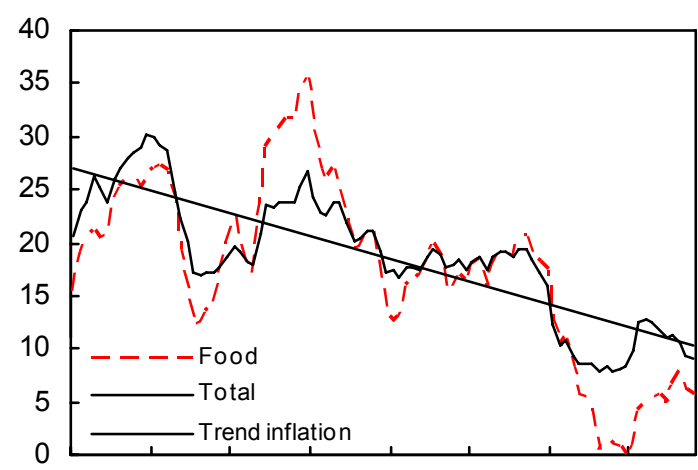

20002001200220032004200520062007

Revenue and Grants and Expenditures, 2000-07 (Percent of GDP, excluding MDRI assistance)

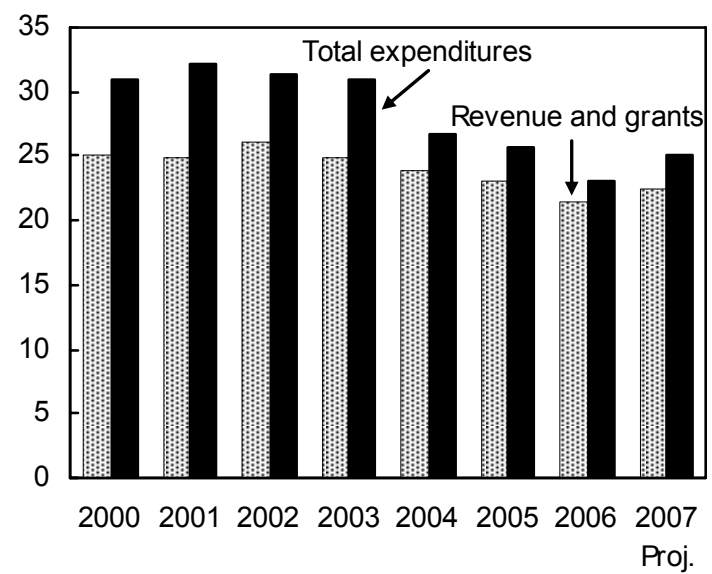

Terms of Trade and Copper Price (2000=100), 2000-07

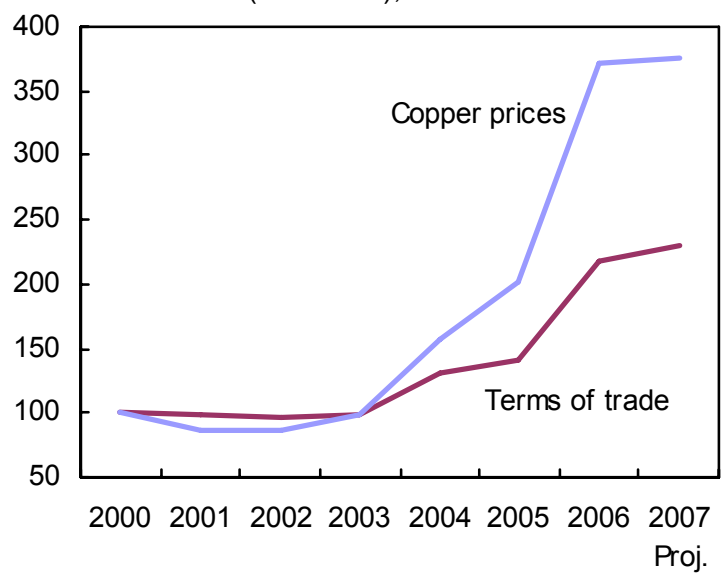


Figure 2. Zambia: Selected Financial Market Indicators

Reserve M oney and Broad M oney January 2001-August 2007

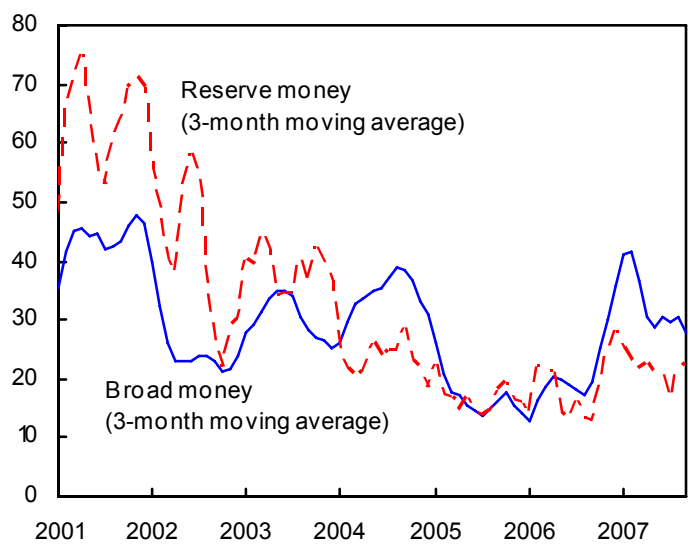

Interest Rates (Percent) January 2004-September 2007

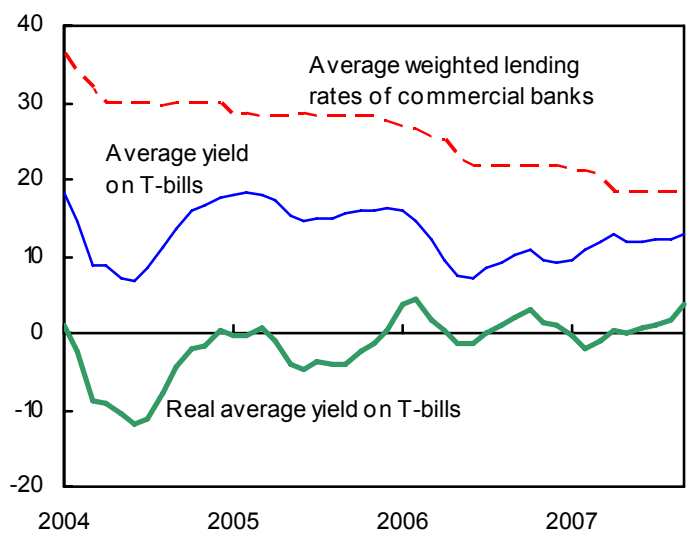

Effective Exchange Rates January 2000-July 2007 V

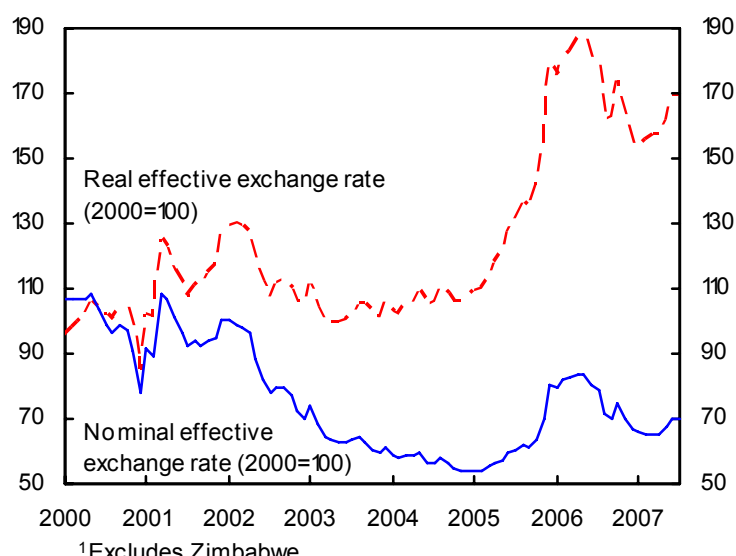

Private Sector Credit, 2001-07

(Annual average)

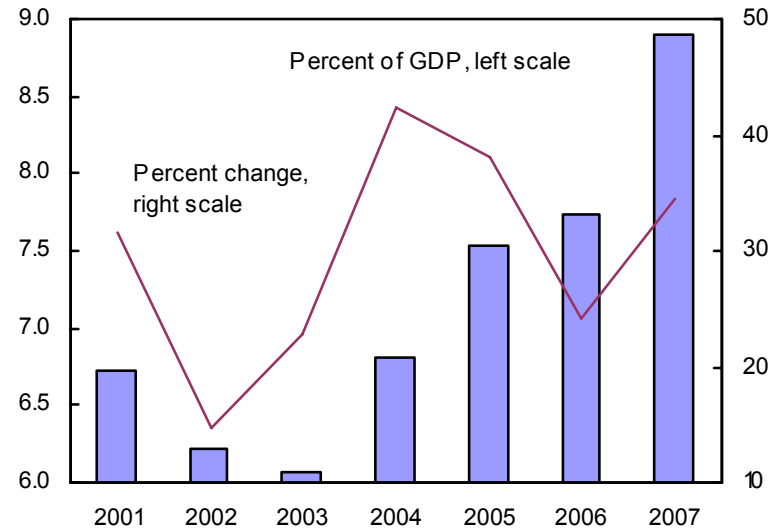

Foreign Investment in Government Securities M arch 2005-September 2007

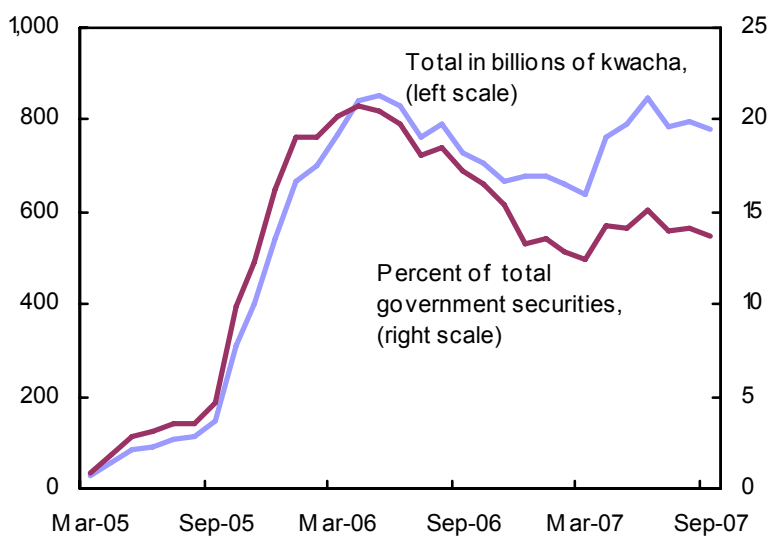

Exchange Rates

January 2004-September 2007

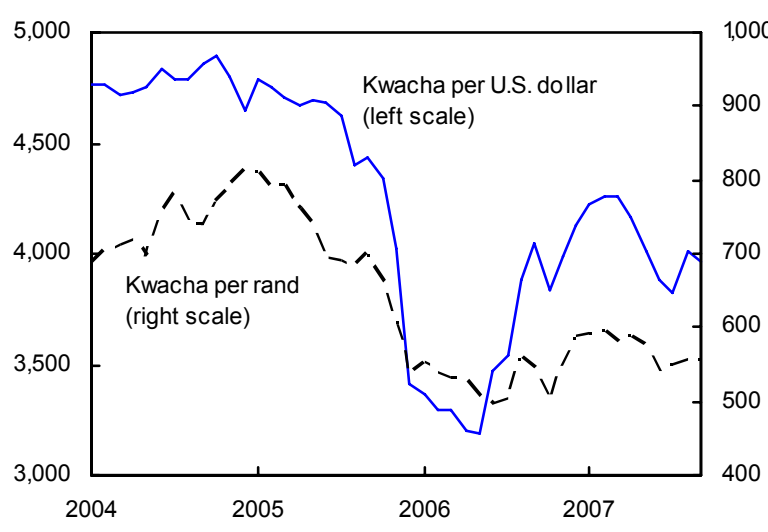


picked up in early 2007, but tighter monetary conditions and a turnaround in the kwacha brought it back to single digits (9 percent) in October.

- $\quad$ Fiscal consolidation continued in 2007, but budget execution has been problematic (Table 2 and 3). Revenue rebounded to outperform projections through September 2007, reflecting higher income tax payments from mining companies. Expenditures were below projections owing to a large shortfall in releases for capital projects and because the released funds were not fully spent due to delays in tender procedures. This underspending resulted in a significant increase in government deposits at the Bank of Zambia (BoZ) and a large discrepancy in the fiscal accounts. The overall balance (including grants) was 0.8 percent of GDP through Septemberabout $2 \frac{1}{2}$ percentage points of GDP higher than projected.

- $\quad$ The rate of monetary expansion has fluctuated widely (Table 4 and Figure 2). Reserve money grew by about 30 percent in 2006 because the BoZ was unable to sterilize fully the sizable foreign exchange purchases. Money growth slowed to 20 percent in June 2007, reflecting intensified open market operations and a tight fiscal position. Reserve money growth again picked up in the third quarter and additional liquidity was injected into the banking system through a reduction in the cash reserve requirement from 14 percent to 8 percent on October 1 . Nominal interest rates on government securities have trended upward since late 2006, while real rates have risen moderately since inflation peaked earlier in the year.

- The exchange rate has been highly volatile in response to variations in the price of copper and shifts in market sentiment toward emerging markets (Figure 2). In late summer 2007, Zambia felt the effects of the increased risk aversion and flight to liquidity that affected global financial markets. The currency came briefly under strong downward pressure as copper prices fell sharply, prompting the BoZ to intervene to maintain orderly market conditions. The kwacha subsequently strengthened as markets settled and copper prices rebounded.

- The banking sector remains sound. It is generally well capitalized, profitable and liquid (Table 6).

- $\quad$ The external position has strengthened considerably (Table 5). The current account deficit (excluding grants) declined markedly to 1.3 percent of GDP in 2006, as copper export receipts doubled and nontraditional exports increased by 30 percent. Copper export receipts remained strong in the first half of 2007. The strong external position and the tight fiscal conditions contributed to a build up of international reserves to about 2.4 months of imports at end-June 2007.

- Notwithstanding progress in some areas, the achievement of the MDGs remains a challenge (Table 7). 


\section{Policy Discussions}

4. Zambia's key medium-term objectives are to further raise growth and enhance income opportunities for the poor while maintaining domestic and external stability. The discussions with the authorities focused on the challenges they face in reconciling the following objectives with domestic and external stability:

- $\quad$ creating fiscal space for increased spending on infrastructure and the social sectors;

- $\quad$ implementing the public expenditure and financial management reforms necessary to strengthen the capacity to undertake the expansion in public investments and to increase overall efficiency;

- managing the macroeconomic effects of foreign exchange inflows from donors and the mining sector; and

- $\quad$ improving conditions for private sector growth.

5. The authorities remain committed to a flexible exchange rate regime, which is characterized as a managed float. Staff analysis indicates that the exchange rate is broadly aligned with macroeconomic fundamentals, including the large improvement in the terms of trade in recent years (Box 1). Despite a large real exchange rate appreciation in recent years, nontraditional export receipts expanded by 30 percent in 2006 and remained strong in the first-half of 2007.

\section{A. Near-Term Policy Challenges and Medium-Term Outlook}

\section{Policies for the rest of $\mathbf{2 0 0 7}$}

6. Incomplete execution of the $\mathbf{2 0 0 7}$ budget through September will challenge fiscal and monetary policy for the rest of the year. With about $1 \frac{1}{2}$ percent of GDP of budgetary releases still unspent by mid-September, full implementation of the budget would result in a large injection of liquidity in the last quarter of the year. The authorities argued that a large proportion of these releases are likely to remain unspent because it will not be possible to carry out all of the planned projects. To ease the burden on monetary policy, however, the authorities will keep releases for the rest of the year below budgeted levels in nonpriority areas. Lower expenditure, together with the overperformance on revenue, is expected to result in net domestic financing for the year as a whole about 2 percentage points of GDP below that budgeted. There is considerable uncertainty about the outcome, however, since ministries may try to spend as much as they can before year end because under newly implemented procedures to reduce the budget carryover from one fiscal year to the next, any unspent funds at year-end must be transferred back to the treasury and new budget authorization sought for the spending. 


\section{Box 1. Zambia: Assessment of External Stability}

A balance of payments position consistent with external stability is one in which (i) the underlying current account is broadly in line with its equilibrium, i.e., there is no fundamental exchange rate misalignment, and (ii) the capital and financial account does not create risks of abrupt shifts in the capital flows.

Current account and fundamental misalignment. Directly estimating Zambia's equilibrium and underlying current account is complicated by data limitations, including the absence of international investment position data, and structural breaks. Moreover, the long-run current account equilibrium (or norm) is difficult to determine in a commodity-dependent economy, particularly one that is undergoing major structural changes, including in its major export base. The exchange rate therefore is assessed by (i) analyzing the relationship between the real effective exchange rate (REER) and a set of fundamental macroeconomic variables; (ii) assessing the exchange rate policy framework; and (iii) assessing the sustainability of domestic financial policies.

- Equilibrium REER. Estimation of the equilibrium REER for the period 19862006 found that the long-run behavior of the REER is explained by the terms of trade and openness to trade. The estimates indicate that the REER appreciation in recent years is consistent with an appreciation of the equilibrium exchange rate, driven mainly by the large increase in copper prices. In the first half of 2007, the REER was 3 percent above the estimated equilibrium REER. Assessment: the current exchange rate does not appear fundamentally misaligned.

- Exchange rate policy. The exchange rate is determined in the interbank foreign exchange market, with intervention by the BoZ confined to keeping market conditions orderly and meeting its international reserves target. Since Zambia is a recipient of sizeable aid inflows, the BoZ can accumulate international reserves without intervening directly in the foreign exchange market if it does not sell all of the aid inflows. Assessment: the exchange rate broadly reflects market conditions and no important market restrictions can be identified.

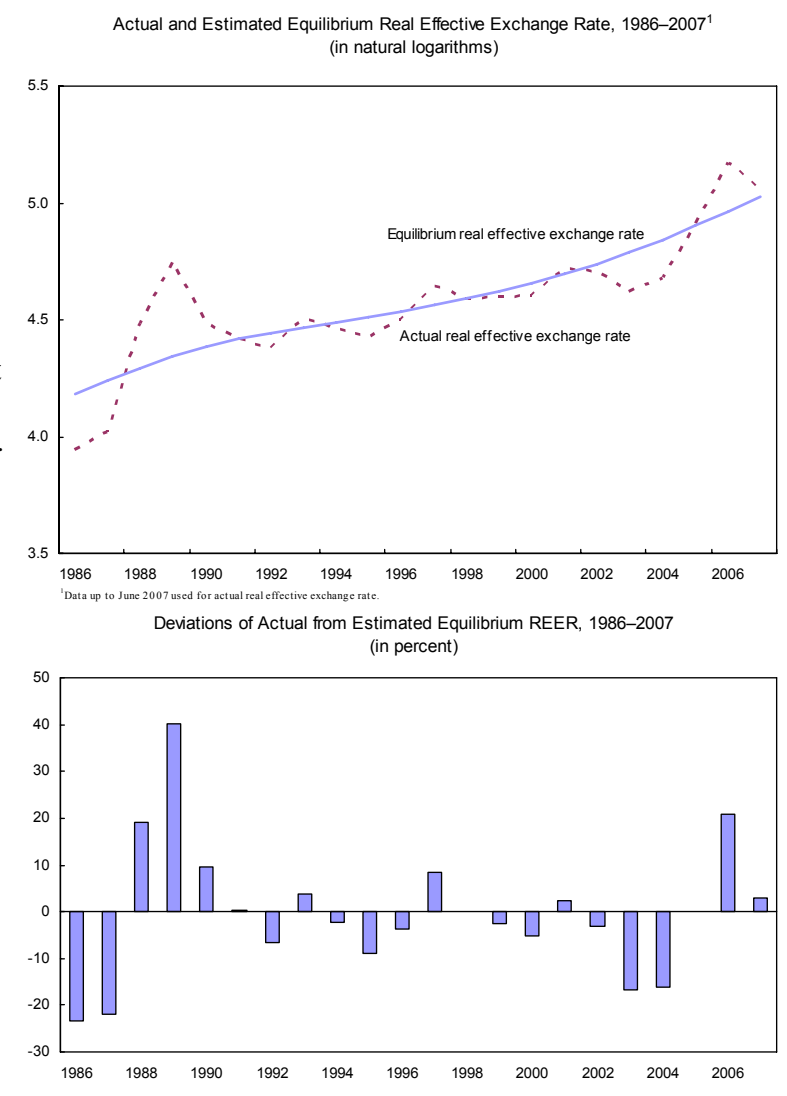

- Domestic policies. The fiscal position is sustainable and is supported by a projected primary deficit of 1.1 percent of GDP in 2007 and expected medium-term real GDP growth of 6 percent. A firm monetary policy has supported a declining trend in inflation. While keeping macroeconomic stability and continuing with structural reforms are expected to generate productivity gains and REER appreciation, all else being equal, the exchange rate adjustment is expected to be very gradual. Assessment: fiscal and monetary policies are consistent with domestic stability and internal balance.

Capital and financial accounts and external stability. Private capital inflows are related primarily to foreign direct investment in mining. Since 2005, some portfolio inflows into public securities have also taken place. However, only about 15 percent of government securities (corresponding to about one-fourth of international reserves) are foreign held and largely in securities with maturities of more than one year. There is no significant secondary market for government securities. Assessment: the current structure of Zambia's capital and financial account suggests a relatively low risk of external instability. 
7. Monetary policy needs to remain firm to contain inflation risks. ${ }^{2}$ Liquidity pressures are likely to pose a challenge in the last few months of 2007 as the pace of government spending picks up. In the staff's view, to contain these pressures, open market operations would have to be stepped up. In addition, the BoZ would have to increase net sales of foreign exchange. The BoZ was concerned, however, that further exchange rate appreciation may constrain its ability to do so in the short-term. Staff therefore expressed serious reservations about the timing, though not the principle, of reducing the statutory reserve requirement. The authorities explained that they aim to offset part of the impact of the lower reserve requirement on liquidity by moving government balances from commercial banks to the BoZ, and that they were prepared to take whatever action was needed to achieve the objectives of the monetary program.

\section{Medium-term outlook}

8. Real GDP is expected to continue to expand at 6-7 percent a year over the medium term (Table 1 and 8). This reflects continued large-scale investments in mining and manufacturing and sustained growth in other sectors. The authorities reiterated their commitment to prudent fiscal and monetary policies designed to bring inflation down gradually to about 5 percent over the next two years. The current account deficit is expected to widen with the projected decline in world copper prices of over 50 percent during the next three years. However, strong export volume growth, from the increased investment in mining capacity, will mitigate the effect from the deterioration in prices. The real exchange rate would tend to depreciate in response to the projected deterioration in the terms of trade. Monetary policy would thus need to remain firm to ensure that the pass-through to import prices of the nominal depreciation does not translate into a rise in the overall rate of inflation. International reserves would increase gradually to $3 \frac{1}{2}$ months of imports by 2010 .

\section{The updated debt sustainability analysis (DSA) finds Zambia at low risk of} debt distress. External debt sustainability has improved substantially thanks to debt relief under the HIPC Initiative and the MDRI. Debt ratios are expected to remain well below the thresholds for both the baseline and alternative scenarios, including one in which large public investment projects in the energy sector involve external financing on nonconcessional terms.

\footnotetext{
${ }^{2}$ The chapter "Monetary Policy and Inflation" in the Selected Issues paper (to be published, www.imf.org) analyzes the link between monetary policy and inflation in Zambia.
} 


\section{B. Creating Fiscal Space ${ }^{3}$}

10. Fiscal space needs to be created to increase spending on infrastructure and the social sectors, as outlined in the FNDP. While pressing for donors to scale up inflows, the authorities will strive to improve revenue collection by strengthening administration and broadening the tax base, restraining nonpriority spending, and making spending more efficient. Discussions with donors suggest that foreign support is not likely to be significantly scaled up (total net inflows are expected to remain at about 5-6 percent of GDP over the medium term). The authorities therefore intend to seek nonconcessional external borrowing for a limited number of projects. Staff agreed that nonconcessional external borrowing could be considered, but only for projects that are demonstrably economically viable and do not jeopardize debt sustainability. Nonconcessional borrowing should only take place if there are well-formulated debt management and project evaluation frameworks in place.

\section{The government's draft medium-term expenditure framework (MTEF)} focuses on creating space for higher capital spending. Revenue is projected to remain broadly stable at 18.4 percent of GDP in 2008 and to rise to 19 percent in 2010, largely owing to strengthened administration. Tax revenue from the mining sector is expected to remain at $1 \frac{1}{2}$ percent of GDP, as in 2007 , though it could increase if the ongoing renegotiations of the mining companies' fiscal terms are successfully completed and additional mines start declaring taxable profits. However, any gains could be negated by a decline in world copper prices. Over time expenditure composition is expected to shift increasingly towards capital spending in line with the authorities' emphasis on investment in infrastructure, but in 2008 the share will stay about the same due to large domestic arrears payments and the need to finance the constitutional review. Wages and salaries are projected to increase significantly due to assumed annual real wage increases of $3-5$ percent. The government intends to clear all current domestic arrears by 2009.

12. The staff largely supported the draft MTEF. It has the right focus on capital investments and arrears payments. The fiscal deficit, excluding grants, is estimated to decline gradually to 5\%/4 percent of GDP in 2010 and domestic financing will hold at about 1 percent of GDP a year. Staff expressed concern about the rise in the wage bill and noted that nominal wage increases should decline in line with projected inflation. They further emphasized the need to speed up the civil service and pay reform. Staff also noted that sound investment planning will be critical to ensuring that the increase in capital outlays is effectively utilized.

13. Reform of the tax system and administration will continue. No major tax policy initiatives are expected in the 2008 budget as efforts are currently focused on renegotiating development agreements with the mining companies. But next year attention is expected to

\footnotetext{
${ }^{3}$ The chapter "Creating Fiscal Space for Implementing the Fifth National Development Plan" in the Selected Issues paper (to be published, www.imf.org) discusses this issue in more detail.
} 
shift to other reforms that will help to achieve the goals of broadening the tax base, unifying rates at more moderate levels, and minimizing the number of exemptions. Consideration should be given to calculating and publishing tax expenditures.

\section{The financial difficulties of the insolvent Public Service Pension Fund (PSPF)}

may leave less fiscal space for investments. The PSPF is a defined-benefit scheme that was closed to new entrants in 2000. The actuarial deficit is estimated at about 10 percent of GDP. As the number of pensioners increases while the membership base declines, the annual cashflow deficit is projected to rise in the medium-term before falling as the scheme winds down (the deficit is estimated at $1 / 2$ percent of GDP over the next few years). The authorities pointed out that the problem relates to too generous benefits that can only be changed through a constitutional amendment. The staff stressed that until another solution on the benefit level is found, budget allocations to the PSPF would need to increase to ensure that pension obligations are met in full. This should be reflected in the revised MTEF and could be partly financed by constraining current expenditures, including moderating the growth in the wage bill.

\section{Public Financial Management and Budget Execution}

\section{Cash management and coordination of fiscal and monetary policy need to be} strengthened. The Ministry of Finance and National Planning (MoFNP) and the BoZ need to cooperate more closely to anticipate and manage emerging liquidity pressures. The planned establishment of a treasury department within MoFNP should improve coordination. The department would prepare cash flow forecasts and coordinate all banking relations between the government and the BoZ. Gradual implementation of both a treasury single account and the IFMIS should further improve cash management.

\section{The government has taken steps to prevent a repeat of this year's difficulties} in budget execution. Execution should improve in 2008 following the Zambia National Tender Board's recent decision that ministries can prepare the necessary papers as soon as the budget is issued to parliament and can submit tenders immediately after budget approval. However, until the budget cycle is brought forward, which the authorities noted is tied up in the constitutional review process, continuing difficulties are likely. ${ }^{4}$

\section{Staff welcomed the authorities' efforts to strengthen debt management. As a}

first step, they have prepared a debt management strategy that describes the existing institutional framework and procedures, and highlights the need to consolidate the legal framework. The mission underscored the importance of implementing the debt management strategy together with measures to improve the capacity to evaluate investment projects.

\footnotetext{
${ }^{4}$ The budget can only be presented to parliament after the fiscal (calendar) year begins and traditionally takes place on the last Friday in January. Parliamentary debate typically takes at least two months.
} 
18. Progress has been made toward signing up to the Extractive Industries

Transparency Initiative (EITI). The mission welcomed the government's efforts, with support from the World Bank, toward formally adopting EITI in a participatory process, in line with EITI guidelines.

\section{Managing Foreign Currency Inflows}

19. The authorities recognized the challenges a booming mining sector and donor inflows pose for monetary policy and international competitiveness. The mission noted that full absorption of aid inflows would require the BoZ to sell foreign exchange on the market to mop up the domestic liquidity generated by spending the inflows. Although the sales would inevitably put upward pressure on the real exchange rate, the BoZ should carefully time them to limit exchange rate volatility. Though the excess liquidity could be mopped up using open market operations, that would tend to raise interest rates and reduce absorption. In view of these effects, the authorities recognized that to promote growth in nonmining sectors it is essential to improve the business environment and boost productivity through, for example, investing in infrastructure and deepening financial markets.

20. The volatility of the Kwacha has at times complicated the conduct of monetary policy. The mission encouraged the authorities to improve the operation of the foreign exchange market, including the need to develop a forward market. Although the performance of the interbank market since its introduction in July 2003 has generally been satisfactory, day-to-day exchange rate volatility has been high. The BoZ recognizes the problem and welcomed the preliminary recommendations of a recent IMF TA mission.

21. The accumulation of gross reserves remains an important objective for the authorities. They expressed their intention to build up reserves to a level equivalent to at least 3 months of imports over the next two years. Standard reserve adequacy measures suggest that Zambia would benefit from some further strengthening of its reserve position. The ratio of reserves to short-term external debt is below the commonly used threshold; however, the ratio of reserves to broad money (M2) is well above the benchmark.

\begin{tabular}{|c|c|c|c|c|c|}
\hline \multicolumn{6}{|c|}{ Implied Reserve Need for Selected African Countries } \\
\hline & \multicolumn{2}{|c|}{ Exports } & \multicolumn{2}{|c|}{ Current Account } & \multirow{3}{*}{$\begin{array}{r}2006 \\
\text { Actual }^{3}\end{array}$} \\
\hline & \multirow{2}{*}{\multicolumn{2}{|c|}{$\begin{array}{r}\text { St. Dev. }{ }^{1} \text { Implied Reserve } \\
\text { Need }^{2}\end{array}$}} & \multicolumn{2}{|c|}{ St. Dev. ${ }^{1}$ Implied Reserve } & \\
\hline & & & & Need $^{2}$ & \\
\hline Cape Verde & 9.3 & 3.8 & 3.4 & 1.4 & 3.8 \\
\hline Ghana & 10.7 & 5.5 & 5.8 & 3.0 & 3.2 \\
\hline Kenya & 3.2 & 2.7 & 2.9 & 2.5 & 3.6 \\
\hline Mozambique & 8.4 & 5.2 & 8.2 & 5.0 & 4.5 \\
\hline Nigeria & 7.6 & 4.9 & 7.9 & 5.1 & 11.8 \\
\hline Senegal & 2.8 & 1.8 & 3.5 & 2.3 & 3.9 \\
\hline Tanzania & 3.7 & 2.9 & 6.1 & 4.7 & 5.3 \\
\hline Uganda & 2.6 & 2.6 & 4.1 & 4.0 & 6.1 \\
\hline Zambia & 3.8 & 2.3 & 5.0 & 3.0 & 2.2 \\
\hline Average & 5.8 & 3.5 & 5.2 & 3.4 & 4.9 \\
\hline 'Standard devi & GDP), 199 & & & & \\
\hline${ }^{2}$ Reserve cov & pports need & a tw & rd deviati & & \\
\hline${ }^{3} \mathrm{Re}$ & & & & & \\
\hline
\end{tabular}
Furthermore, Zambia's level of 
reserves falls somewhat short of the level required to meet a current account shock of two standard deviations (in percent of GDP), but would be sufficient to meet a similar export shock. $^{5}$

\section{E. Improving Conditions for Private Sector Growth}

\section{Improving Zambia's business environment is crucial for enhancing} competitiveness and private sector growth. The discussions focused on three main areas where resolute policy implementation could advance private sector growth: (i) financial sector deepening; (ii) further opening of the trade regime; and (iii) energy sector development.

23. While the banking sector appears sound, more needs to be done to strengthen supervision and broaden the availability of financial services. ${ }^{6}$ The $\mathrm{BoZ}$ is developing a framework for risk-based supervision and implementation of the second phase of the financial sector development plan (FSDP) is going ahead. Over the next year, draft legislation on credit bureau services is expected to be enacted and draft amendments to the Bank of Zambia Act that would make central bank operations more independent, are being prepared. The second FinScope survey on the supply of financial services is being finalized; together with the first survey on demand for financial services, it would provide the basis for policies to expand access to financial services. Regarding the capital market, the authorities will introduce measures to develop an active secondary market in government securities. On the state-owned nonbank financial institutions, the authorities are considering options for making them viable financial intermediaries. Staff emphasized the need for decisive action based on a realistic assessment of their viability.

24. The government is pursuing further trade liberalization. In 2008, Zambia will complete the agreed tariff phase-down for 85 percent of the goods traded within the Southern African Development Community (SADC). Tariffs on the remaining sensitive goods will be eliminated during 2009-12. The authorities estimate the revenue impact to be small in the short term because the value of goods that meet the rules of origin requirements is small. To reduce the cost of trading across borders, the authorities intend to streamline procedures to halve the processing time required for exporting and importing goods.

25. The development of the energy sector is vital to achieving the government's objectives of sustained high growth and poverty reduction. In particular, greater electricity generation will be needed to support the envisaged expansion of mining, private

\footnotetext{
${ }^{5}$ The chapter "Assessing Reserve Adequacy" in the Selected Issues paper (to be published, www.imf.org) provides further details.

${ }^{6}$ The chapter "Progress and Challenges of Financial Sector Reform in Zambia" in the Selected Issues paper (to be published, www.imf.org) provides a discussion of the financial sector reform process.
} 
sector growth, and the planned rural electrification program. Thus, it will be important to move toward full cost recovery of electricity generation, transmission, and distribution. The public utility ZESCO should improve corporate governance and efficiency and gradually adjust the average electricity tariff to long-term marginal cost. Furthermore, to ensure ZESCO's profitability, the mining companies should also pay cost-based electricity rates. Otherwise it will not be possible to undertake the additional investment in capacity needed to meet future demand, including from the mining sector itself.

\section{F. Other Issues}

26. The authorities have made efforts to settle arrears to external private creditors stemming from the past imposition of exchange rate controls. Settlement of the outstanding arrears to commercial creditors was offered on terms similar to those provided by the Paris Club. The authorities are now verifying a small amount of claims outstanding to private individuals. The exchange rate restrictions evidenced by the accumulation of these external payments arrears are subject to Fund approval under Article VIII.

\section{Statistical Issues}

27. Initiatives are underway to improve the quality of economic statistics. The Central Statistical Office (CSO) has launched the very first economic census. It will provide important information about the structure of the Zambian economy, which will be used, among other things, to rebase the national accounts. The CSO is also testing a new, rebased CPI. Compilation of the International Investment Position (IIP) has not yet begun owing to capacity constraints. The authorities expressed their willingness to participate in a project for countries that can publish on their own the Article IV Statistical Appendix. The mission provided assistance to start up the process.

\section{Staff Appraisal}

28. The Zambian authorities continue to pursue sound macroeconomic policies that are contributing to robust growth and bringing inflation down. While the economy is expected to continue to perform well, there is a need to strengthen budget execution and liquidity management - poor execution of the 2007 budget may result in excessive injection of liquidity in the rest of the year, which would complicate the conduct of monetary policy.

29. Monetary policy needs to hold firm to contain inflation risks. This may require that the BoZ, in addition to its open market operations, step up net sales of foreign exchange. The recent lowering of statutory reserve requirements could further increase the pressure on monetary policy in the near term. The decision by the authorities to postpone some of the releases for nonpriority projects in the last quarter and to transfer government deposits at commercial banks to the BoZ will ease the burden on monetary policy. The staff is encouraged by the authorities' assurances to avoid wasteful year-end spending and by the BoZ's commitment to meeting its monetary targets. 
30. The exchange rate and domestic economic and financial policies are consistent with external stability. In particular, the flexible exchange rate policy continues to serve Zambia well. The exchange rate seems to be aligned with economic fundamentals, including large improvement in the terms of trade. The large real appreciation in recent years underscores, however, the need to step up reforms to boost productivity, expand access to credit, and remove impediments to business. Some further modest buildup of international reserves would enhance Zambia's ability to weather external shocks.

31. The authority's development strategy to sustain growth and accelerate poverty reduction faces challenges and risks. Among them are creating the fiscal space for additional spending on infrastructure and the social sectors, implementing public financial management reforms, managing the macroeconomic effects of marked foreign exchange inflows, and improving conditions for private sector growth. Moreover, the limited diversification of the economy heightens potential risks to the economic outlook, most prominently, from a steep decline in copper prices.

\section{The preliminary MTEF and 2008 budget are broadly appropriate. The} targeted increase in revenue and the shift toward capital spending and arrears reduction are welcome, through the projected increase in wages and salaries is a concern. While new hiring in the social sectors is appropriate, there is a need to accelerate civil service and pay reforms, which seem to have stalled.

\section{Better management of public expenditure is essential to ensure that it} generates high returns. Steadfast implementation of the public financial management reforms will be key, in particular, establishing a treasury single account and completing the IFMIS project. It will also be critical to align the budget cycle with the fiscal year.

34. The authorities are working to strengthen debt management. Their approach to external borrowing is appropriately cautious, relying on concessional loans and ensuring that any new borrowing does not undermine debt sustainability. There is, however, a need to complement the debt management strategy with measures to improve capacity to evaluate investment projects.

35. Development of the energy sector is vital to achieving a vibrant private sector and reducing poverty. To this end, it is important to raise tariffs to levels consistent with full cost recovery in electricity generation, transmission, and distribution. There is also a need to strengthen the corporate governance and efficiency of ZESCO.

36. It is expected that the next Article IV consultation will be held on the standard 12month cycle. 
Table 1. Zambia: Selected Economic Indicators

\begin{tabular}{|c|c|c|c|c|c|c|c|}
\hline & 2004 & 2005 & 2006 & $\begin{array}{l}2007 \\
\text { Proj. }\end{array}$ & $\begin{array}{l}2008 \\
\text { Proj. }\end{array}$ & $\begin{array}{l}2009 \\
\text { Proj. }\end{array}$ & $\begin{array}{l}2010 \\
\text { Proj. }\end{array}$ \\
\hline & \multicolumn{7}{|c|}{ (In percent changes; unless otherwise indicated) } \\
\hline \multicolumn{8}{|l|}{ National account and prices } \\
\hline GDP at constant prices & 5.4 & 5.2 & 6.2 & 6.2 & 6.5 & 6.5 & 6.0 \\
\hline GDP deflator & 20.5 & 18.6 & 13.8 & 10.0 & 5.5 & 3.2 & 2.3 \\
\hline $\begin{array}{l}\text { GDP at market prices } \\
\text { (In billions of kwacha) }\end{array}$ & 25,997 & 32,456 & 39,223 & 45,849 & 51,559 & 56,670 & 61,475 \\
\hline \multicolumn{8}{|l|}{ Consumer prices (average) } \\
\hline Headline & 18.0 & 18.3 & 9.0 & 10.8 & 7.3 & 5.9 & 5.0 \\
\hline Underlying (excluding food) & 19.8 & 18.1 & 13.6 & 16.5 & 7.5 & 5.9 & 5.0 \\
\hline Consumer prices (end of period) & 17.5 & 15.9 & 8.2 & 9.0 & 7.0 & 5.0 & 5.0 \\
\hline \multicolumn{8}{|l|}{ External sector } \\
\hline Terms of trade (deterioration -) & 34.6 & 6.8 & 54.8 & 5.4 & -11.3 & -20.3 & -17.5 \\
\hline Average exchange rate (kwacha per U.S. dollar) & 4,779 & 4,464 & 3,601 & $\ldots$ & $\ldots$ & $\ldots$ & $\ldots$ \\
\hline (in percentage change; depreciation -) & -1.0 & 6.6 & 19.3 & $\ldots$ & $\ldots$ & $\ldots$ & $\ldots$ \\
\hline Real effective exchange rate (depreciation -$)^{1}$ & 3.0 & 24.1 & 32.3 & $\ldots$ & $\ldots$ & $\ldots$ & $\ldots$ \\
\hline \multicolumn{8}{|l|}{ Money and credit (end of period) } \\
\hline Domestic credit to the private sector & 47.7 & 18.7 & 54.3 & 35.0 & 21.0 & $\ldots$ & $\ldots$ \\
\hline Reserve money $^{2}$ & 21.1 & 10.2 & 29.7 & -12.6 & 13.1 & $\ldots$ & $\ldots$ \\
\hline M3 & 30.3 & 0.4 & 45.1 & 17.7 & 13.1 & $\ldots$ & $\ldots$ \\
\hline & \multicolumn{7}{|c|}{ (In percent of GDP) } \\
\hline \multicolumn{8}{|l|}{ National accounts } \\
\hline Gross investments & 23.0 & 22.5 & 22.6 & 24.1 & 22.7 & 22.1 & 22.5 \\
\hline Government & 8.7 & 7.0 & 4.1 & 5.7 & 5.8 & 6.3 & 7.5 \\
\hline Private & 14.3 & 15.5 & 18.5 & 18.4 & 16.9 & 15.8 & 15.0 \\
\hline National savings & 16.0 & 17.4 & 25.5 & 20.1 & 21.6 & 20.1 & 19.7 \\
\hline Gross foreign savings & 7.0 & 5.1 & -2.9 & 4.0 & 1.1 & 2.0 & 2.8 \\
\hline \multicolumn{8}{|l|}{ Central government budget ${ }^{3}$} \\
\hline Overall balance & -0.8 & -2.6 & 18.6 & -0.6 & -1.9 & -1.7 & -1.7 \\
\hline (excluding grants) & -6.3 & -8.3 & -7.4 & -5.4 & -6.5 & -5.9 & -5.7 \\
\hline Revenue & 18.2 & 17.4 & 16.9 & 18.4 & 18.4 & 18.7 & 19.0 \\
\hline Grants & 5.5 & 5.6 & 26.0 & 4.9 & 4.6 & 4.1 & 4.0 \\
\hline Total expenditure ${ }^{4}$ & 24.5 & 25.7 & 24.3 & 23.8 & 24.8 & 24.5 & 24.7 \\
\hline \multicolumn{8}{|l|}{ External sector } \\
\hline \multicolumn{8}{|l|}{ Current account balance } \\
\hline (including official grants) & -7.0 & -5.1 & 2.9 & -4.0 & -1.1 & -2.0 & -2.8 \\
\hline \multirow[t]{2}{*}{ (excluding official grants) } & -12.2 & -10.9 & -1.3 & -8.8 & -6.1 & -6.8 & -7.8 \\
\hline & \multicolumn{7}{|c|}{ (In percent of export of goods and services) } \\
\hline NPV of external public debt (including IMF) & 175.5 & 80.1 & 16.0 & 13.7 & 14.3 & 17.0 & 20.1 \\
\hline
\end{tabular}

Sources: Zambian authorities; and IMF staff estimates and projections.

${ }^{1}$ Excludes Zimbabwe.

${ }^{2}$ The projected reduction in reserve money for December 2007 reflects the lowering of statutory reserve requirements from 14 to 8 percent on October 1, 2007.

${ }^{3}$ Grants in 2006 include MDRI debt cancellation amounting to 21.4 percent of GDP.

${ }^{4}$ Including discrepancy between the above-the-line balance and below-the-line financing. 
Table 2. Zambia: Fiscal Operations of the Central Government (In billions of kwacha)

\begin{tabular}{|c|c|c|c|c|c|c|c|c|c|}
\hline & \multirow[t]{2}{*}{2005} & \multirow[t]{2}{*}{2006} & \multicolumn{4}{|c|}{2007} & \multirow{2}{*}{$\begin{array}{l}2008 \\
\text { Proj. }\end{array}$} & \multirow{2}{*}{$\begin{array}{l}2009 \\
\text { Proj. }\end{array}$} & \multirow{2}{*}{$\begin{array}{r}2010 \\
\text { Proj. }\end{array}$} \\
\hline & & & $\begin{array}{r}\text { Jan-Sep } \\
\text { Budget } \\
\end{array}$ & $\begin{array}{r}\text { Jan-Sep } \\
\text { Act. } \\
\end{array}$ & Budget & Proj. & & & \\
\hline Revenue and grants & 7,467 & 16,825 & 7,838 & 7,968 & 10,306 & 10,648 & 11,848 & 12,923 & 14,137 \\
\hline Revenue & 5,642 & 6,618 & 6,018 & 6,347 & 8,125 & 8,422 & 9,467 & 10,573 & 11,661 \\
\hline Tax & 5,512 & 6,317 & 5,808 & 6,076 & 7,807 & 8,059 & 9,075 & 10,142 & 11,193 \\
\hline Income taxes & 2,455 & 2,960 & 2,640 & 2,899 & 3,491 & 3,743 & 4,219 & 4,696 & 5,126 \\
\hline Value-added tax & 1,633 & 1,792 & 1,718 & 1,656 & 2,339 & 2,339 & 2,666 & 2,992 & 3,338 \\
\hline Excise taxes & 768 & 821 & 808 & 850 & 1,104 & 1,104 & 1,243 & 1,394 & 1,568 \\
\hline Customs duties & 656 & 744 & 642 & 672 & 874 & 874 & 949 & 1,060 & 1,162 \\
\hline Nontax & 130 & 301 & 210 & 271 & 318 & 363 & 392 & 431 & 467 \\
\hline Grants & 1,825 & 10,207 & 1,819 & 1,621 & 2,180 & 2,226 & 2,380 & 2,350 & 2,476 \\
\hline Budget support & 543 & 423 & 582 & 379 & 582 & 575 & 614 & 678 & 692 \\
\hline Project grants & 1,282 & 1,374 & 1,237 & 1,242 & 1,598 & 1,651 & 1,766 & 1,672 & 1,784 \\
\hline Debt reduction (including MDRI) & 0 & 8,410 & 0 & 0 & 0 & 0 & 0 & 0 & 0 \\
\hline Expenditures & 8,350 & 9,051 & 8,551 & 8,320 & 11,546 & 11,317 & 12,806 & 13,895 & 15,191 \\
\hline Current expenditures & 5,828 & 7,203 & 6,111 & 6,148 & 8,380 & 8,371 & 9,378 & 10,027 & 10,552 \\
\hline Wages and salaries & 2,455 & 2,833 & 2,599 & 2,545 & 3,535 & 3,547 & 4,240 & 4,806 & 5,319 \\
\hline Goods and services & 1,249 & 1,779 & 1,248 & 1,219 & 1,744 & 1,673 & 2,108 & 2,080 & 2,053 \\
\hline Interest payments & 865 & 749 & 493 & 548 & 705 & 765 & 687 & 735 & 719 \\
\hline Other & 1,259 & 1,842 & 1,771 & 1,837 & 2,396 & 2,386 & 2,343 & 2,407 & 2,461 \\
\hline Capital expenditure & 2,267 & 1,601 & 2,231 & 1,966 & 2,827 & 2,607 & 2,976 & 3,557 & 4,638 \\
\hline Of which: domestically financed & 565 & 599 & 1,086 & 781 & 1,350 & 1,032 & 1,340 & 1,991 & 2,896 \\
\hline Domestic arrears payments & 254 & 247 & 208 & 206 & 339 & 339 & 451 & 311 & 0 \\
\hline Discrepancy (-overfinancing) ${ }^{1}$ & 24 & -483 & -139 & 704 & 0 & 400 & 0 & 0 & 0 \\
\hline \multicolumn{10}{|l|}{ Overall balance } \\
\hline Including grants & -858 & 7,291 & -852 & 352 & $-1,241$ & -269 & -958 & -972 & $-1,054$ \\
\hline Excluding grants & $-2,683$ & $-2,916$ & $-2,671$ & $-1,269$ & $-3,421$ & $-2,495$ & $-3,338$ & $-3,322$ & $-3,530$ \\
\hline Financing & 858 & $-7,291$ & 852 & -352 & 1,241 & 269 & 958 & 972 & 1,054 \\
\hline External financing (net) & 241 & $-6,225$ & 407 & 248 & 498 & 484 & 339 & 405 & 439 \\
\hline Of which: budget support & 84 & 36 & 167 & 0 & 167 & 121 & 81 & 123 & 123 \\
\hline Of which: debt reduction (MDRI) & 0 & $-6,392$ & 0 & 0 & 0 & 0 & 0 & 0 & 0 \\
\hline Domestic financing (net) & 617 & $-1,066$ & 445 & -601 & 743 & -215 & 619 & 567 & 615 \\
\hline Bank financing & -96 & $-1,806$ & 60 & -868 & 100 & -489 & 100 & 100 & 100 \\
\hline Of which: BoZ onlending of IMF MDRI relief & 0 & $-2,018$ & 0 & 0 & 0 & 0 & 0 & 0 & 0 \\
\hline Nonbank financing & 713 & 740 & 385 & 268 & 643 & 274 & 519 & 467 & 515 \\
\hline \multicolumn{10}{|l|}{ Memorandum items: } \\
\hline Primary balance, including grants & 6 & 8,041 & -359 & 900 & -536 & 497 & -270 & -237 & -335 \\
\hline Poverty-reducing spending ${ }^{2}$ & 1,995 & 2,356 & & 2,524 & 4,096 & 3,793 & & & \\
\hline External budget support & 628 & 459 & 750 & 379 & 750 & 697 & 695 & 801 & 815 \\
\hline Stock of domestic arrears & 947 & 1,028 & 670 & & 539 & 762 & 311 & 0 & 0 \\
\hline Stock of domestic government securities & 5,271 & 6,242 & 6,687 & 6,811 & 6,984 & 6,906 & 7,524 & 8,091 & 8,706 \\
\hline MDRI debt sevice savings & $\ldots$ & 94 & $\ldots$ & $\ldots$ & $\ldots$ & 245 & 359 & 406 & $\ldots$ \\
\hline
\end{tabular}

Sources: Zambian authorities; and IMF staff estimates and projections.

${ }^{1}$ Discrepancy largely reflects the net change in releases to line ministries that are not fully executed by the end of the year and thus are not reflected in the domestic financing figures until the following year.

${ }^{2}$ Excluding donor financed projects. 
Table 3. Zambia: Fiscal Operations of the Central Government (In percent of GDP)

\begin{tabular}{|c|c|c|c|c|c|c|c|c|c|}
\hline & \multirow[t]{2}{*}{2005} & \multirow[t]{2}{*}{2006} & \multicolumn{4}{|c|}{2007} & \multirow{2}{*}{$\begin{array}{l}2008 \\
\text { Proj. }\end{array}$} & \multirow{2}{*}{$\begin{array}{l}2009 \\
\text { Proj. }\end{array}$} & \multirow{2}{*}{$\begin{array}{l}2010 \\
\text { Proj. }\end{array}$} \\
\hline & & & $\begin{array}{r}\text { Jan-Sep } \\
\text { Budget }\end{array}$ & $\begin{array}{r}\text { Jan-Sep } \\
\text { Act. }\end{array}$ & Budget & Proj. & & & \\
\hline Revenue and grants & 23.0 & 42.9 & 17.1 & 17.4 & 22.5 & 23.2 & 23.0 & 22.8 & 23.0 \\
\hline Revenue & 17.4 & 16.9 & 13.1 & 13.8 & 17.7 & 18.4 & 18.4 & 18.7 & 19.0 \\
\hline Tax & 17.0 & 16.1 & 12.7 & 13.3 & 17.0 & 17.6 & 17.6 & 17.9 & 18.2 \\
\hline Income taxes & 7.6 & 7.5 & 5.8 & 6.3 & 7.6 & 8.2 & 8.2 & 8.3 & 8.3 \\
\hline Value-added tax & 5.0 & 4.6 & 3.7 & 3.6 & 5.1 & 5.1 & 5.2 & 5.3 & 5.4 \\
\hline Excise taxes & 2.4 & 2.1 & 1.8 & 1.9 & 2.4 & 2.4 & 2.4 & 2.5 & 2.5 \\
\hline Customs duties & 2.0 & 1.9 & 1.4 & 1.5 & 1.9 & 1.9 & 1.8 & 1.9 & 1.9 \\
\hline Nontax & 0.4 & 0.8 & 0.5 & 0.6 & 0.7 & 0.8 & 0.8 & 0.8 & 0.8 \\
\hline Grants & 5.6 & 26.0 & 4.0 & 3.5 & 4.8 & 4.9 & 4.6 & 4.1 & 4.0 \\
\hline Budget support & 1.7 & 1.1 & 1.3 & 0.8 & 1.3 & 1.3 & 1.2 & 1.2 & 1.1 \\
\hline Project grants & 3.9 & 3.5 & 2.7 & 2.7 & 3.5 & 3.6 & 3.4 & 3.0 & 2.9 \\
\hline Debt reduction (including MDRI) & 0.0 & 21.4 & 0.0 & 0.0 & 0.0 & 0.0 & 0.0 & 0.0 & 0.0 \\
\hline Expenditures & 25.7 & 23.1 & 18.6 & 18.1 & 25.2 & 24.7 & 24.8 & 24.5 & 24.7 \\
\hline Current expenditures & 18.0 & 18.4 & 13.3 & 13.4 & 18.3 & 18.3 & 18.2 & 17.7 & 17.2 \\
\hline Wages and salaries & 7.6 & 7.2 & 5.7 & 5.6 & 7.7 & 7.7 & 8.2 & 8.5 & 8.7 \\
\hline Goods and services & 3.8 & 4.5 & 2.7 & 2.7 & 3.8 & 3.6 & 4.1 & 3.7 & 3.3 \\
\hline Interest payments & 2.7 & 1.9 & 1.1 & 1.2 & 1.5 & 1.7 & 1.3 & 1.3 & 1.2 \\
\hline Other & 3.9 & 4.7 & 3.9 & 4.0 & 5.2 & 5.2 & 4.5 & 4.2 & 4.0 \\
\hline Capital expenditure & 7.0 & 4.1 & 4.9 & 4.3 & 6.2 & 5.7 & 5.8 & 6.3 & 7.5 \\
\hline Of which: domestically financed & 1.7 & 1.5 & 2.4 & 1.7 & 2.9 & 2.3 & 2.6 & 3.5 & 4.7 \\
\hline Domestic arrears payments & 0.8 & 0.6 & 0.5 & 0.4 & 0.7 & 0.7 & 0.9 & 0.5 & 0.0 \\
\hline Discrepancy (-overfinancing) ${ }^{1}$ & 0.1 & -1.2 & -0.3 & 1.5 & 0.0 & 0.9 & 0.0 & 0.0 & 0.0 \\
\hline \multicolumn{10}{|l|}{ Overall balance } \\
\hline Including grants & -2.6 & 18.6 & -1.9 & 0.8 & -2.7 & -0.6 & -1.9 & -1.7 & -1.7 \\
\hline Excluding grants & -8.3 & -7.4 & -5.8 & -2.8 & -7.5 & -5.4 & -6.5 & -5.9 & -5.7 \\
\hline Financing & 2.6 & -18.6 & 1.9 & -0.8 & 2.7 & 0.6 & 1.9 & 1.7 & 1.7 \\
\hline External financing (net) & 0.7 & -15.9 & 0.9 & 0.5 & 1.1 & 1.1 & 0.7 & 0.7 & 0.7 \\
\hline Of which: budget support & 0.3 & 0.1 & 0.4 & 0.0 & 0.4 & 0.3 & 0.2 & 0.2 & 0.2 \\
\hline Of which: debt reduction (MDRI) & $\ldots$ & -16.3 & 0.0 & 0.0 & 0.0 & 0.0 & 0.0 & 0.0 & 0.0 \\
\hline Domestic financing (net) & 1.9 & -2.7 & 1.0 & -1.3 & 1.6 & -0.5 & 1.2 & 1.0 & 1.0 \\
\hline Bank financing & -0.3 & -4.6 & 0.1 & -1.9 & 0.2 & -1.1 & 0.2 & 0.2 & 0.2 \\
\hline Of which: Onlending of IMF MDRI relief & 0.0 & -5.1 & 0.0 & 0.0 & 0.0 & 0.0 & 0.0 & 0.0 & 0.0 \\
\hline Nonbank financing & 2.2 & 1.9 & 0.8 & 0.6 & 1.4 & 0.6 & 1.0 & 0.8 & 0.8 \\
\hline \multicolumn{10}{|l|}{ Memorandum items: } \\
\hline Primary balance, including grants & 0.0 & 20.5 & -0.8 & 2.0 & -1.2 & 1.1 & -0.5 & -0.4 & -0.5 \\
\hline Poverty-reducing spending $^{2}$ & 6.1 & 6.0 & $\ldots$ & 5.5 & 8.9 & 8.3 & $\ldots$ & $\ldots$ & $\ldots$ \\
\hline External budget support & 1.9 & 1.2 & 1.6 & 0.8 & 1.6 & 1.5 & 1.3 & 1.4 & 1.3 \\
\hline Stock of domestic arrears & 2.9 & 2.6 & 1.8 & $\ldots$ & 1.2 & 1.7 & 0.6 & 0.0 & 0.0 \\
\hline Stock of domestic government securities & 16.2 & 15.9 & 14.6 & 14.9 & 15.2 & 15.1 & 14.6 & 14.3 & 14.2 \\
\hline MDRI debt sevice savings & & 0.2 & $\ldots$ & $\ldots$ & & 0.5 & 0.7 & 0.7 & \\
\hline Nominal GDP (in billions of kwacha) & 32,456 & 39,223 & 45,849 & 45,849 & 45,849 & 45,849 & 51,559 & 56,670 & 61,475 \\
\hline
\end{tabular}

Sources: Zambian authorities; and IMF staff estimates and projections.

${ }^{1}$ Discrepancy largely reflects the net change in releases to line ministries that are not fully executed by the end of the year and thus are not reflected in the domestic financing figures until the following year.

${ }^{2}$ Excluding donor financed projects. 
Table 4. Zambia: Monetary Accounts ${ }^{1}$ (In billions of kwacha, unless otherwise indicated)

\begin{tabular}{|c|c|c|c|c|c|c|}
\hline & \multirow{2}{*}{2005} & \multicolumn{2}{|c|}{2006} & \multicolumn{2}{|c|}{2007} & \multirow{2}{*}{$\begin{array}{l}2008 \\
\text { Proj. }\end{array}$} \\
\hline & & $\begin{array}{l}\text { Jun. } \\
\text { Act. }\end{array}$ & $\begin{array}{l}\text { Dec. } \\
\text { Act. }\end{array}$ & $\begin{array}{l}\text { Jun. } \\
\text { Act. }\end{array}$ & $\begin{array}{l}\text { Dec. } \\
\text { Proj. }\end{array}$ & \\
\hline & \multicolumn{6}{|c|}{ I. Monetary Survey } \\
\hline Net foreign assets & -282 & 1,666 & 3,953 & 3,947 & 4,420 & 5,425 \\
\hline Net domestic assets & 6,123 & 4,623 & 4,524 & 4,610 & 5,555 & 5,858 \\
\hline Claims on general government (net) & 2,387 & 2,383 & 2,374 & 1,492 & 1,927 & 2,027 \\
\hline Of which: Central government (net) & 2,416 & 2,404 & 2,440 & 1,515 & 1,950 & 2,050 \\
\hline Claims on private sector & 2,437 & 2,942 & 3,760 & 4,302 & 5,076 & 6,139 \\
\hline Claims on public enterprises & 230 & 560 & 224 & 246 & 246 & 300 \\
\hline Other items (net) ${ }^{2}$ & 1,068 & $-1,263$ & $-1,834$ & $-1,430$ & $-1,693$ & $-2,609$ \\
\hline Money and quasi-money (M3) & 5,841 & 6,289 & 8,477 & 8,557 & 9,975 & 11,282 \\
\hline Broad money (M2) & 3,860 & 4,281 & 5,763 & 5,513 & $\ldots$ & $\ldots$ \\
\hline \multirow[t]{2}{*}{ Foreign exchange deposits } & 1,981 & 2,008 & 2,714 & 3,044 & $\ldots$ & $\ldots$ \\
\hline & \multicolumn{6}{|c|}{ II. Bank of Zambia } \\
\hline Net foreign assets & $-1,235$ & 1,283 & 2,807 & 3,120 & 3,337 & 4,325 \\
\hline Of which: IMF (net) & $-3,057$ & -110 & -138 & -277 & -338 & -366 \\
\hline Net domestic assets & 3,132 & 645 & -348 & -798 & $-1,187$ & $-1,893$ \\
\hline Claims on central government (net) & 1,070 & 593 & 844 & -140 & -184 & -184 \\
\hline Claims on nongovernment & 375 & 617 & 309 & 337 & 338 & 340 \\
\hline Claims on private sector & 53 & 52 & 47 & 56 & 58 & 60 \\
\hline Claims on public enterprises & 96 & 343 & 0 & 0 & 0 & 0 \\
\hline Claims on commercial banks & 226 & 222 & 262 & 280 & 280 & 280 \\
\hline Other items (net) ${ }^{2}$ & 1,687 & -565 & $-1,502$ & -995 & $-1,341$ & $-2,050$ \\
\hline Of which: Open market operations & -401 & -379 & -766 & -697 & -800 & $\ldots$ \\
\hline Reserve money ${ }^{3}$ & 1,896 & 1,928 & 2,459 & 2,321 & 2,150 & 2,431 \\
\hline Currency outside banks and cash in vaults & 962 & 999 & 1,224 & 1,329 & $\ldots$ & $\ldots$ \\
\hline Commercial bank deposits & 926 & 924 & 1,226 & 986 & $\ldots$ & $\ldots$ \\
\hline
\end{tabular}

Memorandum items:

(12-month percentage change over beginning period broad money)

Monetary survey:

Net foreign assets

Net domestic assets

Claims on central government (net)

Claims on private sector

$\begin{array}{rrrrrr}29.0 & 81.3 & 72.5 & 36.2 & 5.5 & 10.1 \\ -28.6 & -74.6 & -27.4 & -0.2 & 12.2 & 3.0 \\ -1.7 & -5.3 & 0.4 & -13.7 & -5.0 & 1.0 \\ 6.6 & 3.9 & 22.6 & 21.6 & 15.5 & 10.7\end{array}$

(In 12-month percentage change; unless otherwise indicated)

$\begin{array}{rrrrrr}10.2 & 1.6 & 29.7 & 20.4 & -12.6 & 13.1 \\ 0.4 & 6.6 & 45.1 & 36.1 & 17.7 & 13.1 \\ 18.7 & 8.3 & 54.3 & 46.2 & 35.0 & 21.0 \\ 18.0 & 16.0 & 21.6 & 18.7 & 21.8 & 21.9 \\ 3.1 & 3.3 & 3.4 & 3.7 & 4.6 & 4.6 \\ 7.5 & 7.5 & 9.6 & 9.4 & 11.1 & 11.9 \\ 331 & 393 & 595 & 816 & 850 & 1,007 \\ 3,509 & 3,569 & 4,407 & 3,771 & \ldots & \ldots\end{array}$

Sources: Zambian authorities; and Fund staff estimates and projections.

${ }^{1}$ End of period.

${ }^{2}$ Include valuation and HIPC Initiative Account (balances were K3,253 billion and K2,209 billion at end-2005 and end-2006, respectively).

${ }^{3}$ The projected reduction in reserve money for December 2007 reflects the lowering of statutory reserve requirements 
Table 5. Zambia: Balance of Payments

(In millions of U.S. dollars, unless otherwise indicated)

\begin{tabular}{|c|c|c|c|c|c|c|}
\hline & 2005 & 2006 & $\begin{array}{c}2007 \\
\text { Proj. }\end{array}$ & $\begin{array}{c}2008 \\
\text { Proj. }\end{array}$ & $\begin{array}{c}2009 \\
\text { Proj. }\end{array}$ & $\begin{array}{l}2010 \\
\text { Proj. }\end{array}$ \\
\hline Current account & -662 & 120 & -699 & -442 & -540 & -680 \\
\hline Trade balance & 50 & 1,183 & 970 & 961 & 437 & 32 \\
\hline Exports, f.o.b. & 2,210 & 3,819 & 4,258 & 4,406 & 4,043 & 3,694 \\
\hline Of which: copper & 1,486 & 2,938 & 3,159 & 3,274 & 2,917 & 2,530 \\
\hline Imports, f.o.b & $-2,161$ & $-2,636$ & $-3,288$ & $-3,444$ & $-3,606$ & $-3,662$ \\
\hline Of which: oil & -312 & -454 & -483 & -532 & -560 & -592 \\
\hline Services (net) & -199 & -284 & -591 & -437 & -312 & -262 \\
\hline Income (net) & -619 & -960 & $-1,294$ & $-1,151$ & -843 & -607 \\
\hline Of which: interest on public debt & -110 & -17 & -7 & -13 & -12 & -11 \\
\hline Current transfers (net) & 107 & 180 & 215 & 184 & 178 & 157 \\
\hline Budget support grants & 131 & 116 & 142 & 154 & 170 & 173 \\
\hline Sector-wide approach grants & 0 & 143 & 154 & 128 & 126 & 118 \\
\hline Private transfers & -24 & -80 & -82 & -97 & $\begin{array}{r}-118 \\
21\end{array}$ & -134 \\
\hline Capital and financial account & 704 & 1,013 & 1,063 & 601 & 698 & 839 \\
\hline Capital account & 287 & 2,600 & 252 & 314 & 292 & 328 \\
\hline Project grants & 287 & 197 & 252 & 314 & 292 & 328 \\
\hline External debt cancellation & 0 & 2,403 & 0 & 0 & 0 & 0 \\
\hline Financial account & 417 & $-1,587$ & 811 & 288 & 406 & 510 \\
\hline Foreign direct and portfolio investments & 497 & 559 & 909 & 636 & 421 & 383 \\
\hline Other investments & -80 & $-2,146$ & -98 & -348 & -15 & 128 \\
\hline Medium and long-term & -80 & $-1,838$ & 85 & -154 & 157 & 150 \\
\hline Public sector (net) & -65 & $-1,779$ & 114 & 85 & 101 & 110 \\
\hline Disbursements & 160 & 91 & 166 & 116 & 130 & 138 \\
\hline Of which: budget support & 24 & 9 & 30 & 20 & 31 & 31 \\
\hline Amortization due & -225 & $-1,870$ & -52 & -31 & -29 & -28 \\
\hline Commercial banks (net) & 91 & 62 & -65 & 0 & 0 & 0 \\
\hline Other sectors & -106 & -121 & 36 & -239 & 56 & 40 \\
\hline Short-term & 0 & -308 & -183 & -194 & -172 & -22 \\
\hline Errors and omissions & -172 & -311 & -150 & 0 & 0 & 0 \\
\hline Overall balance & -129 & 821 & 213 & 159 & 158 & 159 \\
\hline \multicolumn{7}{|l|}{ Financing } \\
\hline Central bank net reserves (- increase) & -351 & -821 & -213 & -159 & -158 & -159 \\
\hline Of which: gross reserve change & -109 & -264 & -255 & -159 & -158 & -159 \\
\hline Of which: Use of Fund loans & -236 & -557 & 42 & 0 & 0 & -1 \\
\hline Exceptional financing & 480 & 0 & 0 & 0 & 0 & 0 \\
\hline Financing gap & 0 & 0 & 0 & 0 & 0 & 0 \\
\hline \multicolumn{7}{|l|}{ Memorandum items: } \\
\hline Current account including capital grants (in percent of GDP) & -5.1 & 2.9 & -4.0 & -1.1 & -2.0 & -2.8 \\
\hline Current account excluding grants (in percent of GDP) & -10.9 & -1.3 & -8.8 & -6.1 & -6.8 & -7.8 \\
\hline Copper export volume (thousands of metric tons) & 439 & 476 & 476 & 559 & 664 & 740 \\
\hline Copper export price (U.S. dollars per pound) & 1.5 & 2.8 & 3.0 & 2.7 & 2.0 & 1.5 \\
\hline Net creditor/donor support (in percent of GDP) ${ }^{1}$ & 6.7 & 4.2 & 6.0 & 5.4 & 5.3 & 5.5 \\
\hline Of which: official grants & 5.7 & 4.2 & 4.9 & 5.0 & 4.8 & 5.0 \\
\hline Gross international reserves & 331 & 595 & 850 & 1,009 & 1,167 & 1,326 \\
\hline In months of imports & 1.5 & 2.2 & 2.4 & 2.8 & 3.2 & 3.6 \\
\hline GDP (in millions of U.S. dollars) & 7,271 & 10,893 & 11,261 & 11,884 & 12,212 & 12,408 \\
\hline
\end{tabular}

Sources: Zambian authorities; and Fund staff estimates and projections.

${ }^{1}$ Defined as disbursements, plus grants, less debt service. 
Table 6. Zambia: Financial Soundness Indicators, 2002-07

(In percent, unless otherwise indicated)

\begin{tabular}{|c|c|c|c|c|c|c|}
\hline & 2002 & 2003 & 2004 & 2005 & 2006 & $\begin{array}{c}2007 \\
\text { Jun }\end{array}$ \\
\hline \multicolumn{7}{|l|}{ Capital adequacy } \\
\hline Regulatory capital to risk-weighted assets & 28.0 & 23.0 & 22.2 & 28.4 & 20.4 & 18.9 \\
\hline Tier 1 regulatory capital to risk-weighted assets & 24.8 & 21.0 & 19.6 & 26.2 & 18 & 16.5 \\
\hline Capital to total assets & 12.3 & 11.0 & 9.7 & 11.6 & 9.1 & 9.9 \\
\hline \multicolumn{7}{|l|}{ Asset quality } \\
\hline Past due advances (NPL) to total advances & 11.4 & 5.3 & 7.6 & 8.9 & 11.3 & 8.5 \\
\hline Loan loss provisions to nonperforming loans & 73.9 & 89.3 & 102.8 & 90.7 & 83.3 & 104.9 \\
\hline Bad debt provisions to advances & 8.4 & 4.7 & 7.8 & 5.4 & 6.8 & 7.2 \\
\hline \multicolumn{7}{|l|}{ Loan concentration ${ }^{1}$} \\
\hline Households & 12.3 & 17.5 & 13.1 & 19.5 & 16.1 & 16.8 \\
\hline Government and parastatals & 6.4 & 4.3 & 5.5 & 5.5 & 6.2 & 5.6 \\
\hline Agriculture & 22.2 & 24.7 & 30.3 & 27.0 & 23.9 & 23.0 \\
\hline Mining & 3.6 & 4.9 & 4.5 & 4.0 & 4.4 & 5.0 \\
\hline Manufacturing & 14.0 & 12.7 & 13.0 & 12.0 & 14 & 11.0 \\
\hline Construction & 1.6 & 2.2 & 2.0 & 2.0 & 2.4 & 3.0 \\
\hline Services & 8.5 & 7.3 & 8.1 & 7.0 & 9.8 & 9.0 \\
\hline Others & 49.9 & 45.1 & 42.0 & 48.0 & 45.5 & 49.0 \\
\hline \multicolumn{7}{|l|}{ Earnings and profitability } \\
\hline Return on average assets & 6.5 & 5.4 & 3.1 & 6.5 & 5.1 & 4.7 \\
\hline Return on equity & 52.8 & 48.5 & 29.8 & 46.4 & 30.6 & 30.9 \\
\hline Gross interest income to total gross income & 65.8 & 66.1 & 78.2 & 60.9 & 60.2 & 58.6 \\
\hline Gross noninterest income to total gross income & 34.2 & 33.9 & 46.3 & 39.1 & 39.8 & 41.1 \\
\hline Net interest margin & 15.3 & 13.2 & 11.8 & 11.8 & 12.8 & 10.9 \\
\hline \multicolumn{7}{|l|}{ Liquidity $^{2}$} \\
\hline Liquid assets to total assets & 78.6 & 74.7 & 66.6 & 41.0 & 41.3 & 36.5 \\
\hline Liquid assets to total deposits & 69.7 & 73.5 & 73.7 & 51.0 & 49.6 & 44.7 \\
\hline Advances to deposits ratio & 29.9 & 33.3 & 37.3 & 44.5 & 49 & 56.6 \\
\hline \multicolumn{7}{|l|}{ Exposure to foreign currency } \\
\hline Foreign currency loans to total gross loans & 42.8 & 46.8 & 41.2 & 36.2 & 34.0 & 30.3 \\
\hline Foreign currency liabilities to total liabilities & 62.2 & 58.4 & 58.4 & 31.0 & 61.2 & \\
\hline Net open position in foreign exchange to capital & 15.7 & 10.0 & 10.0 & 2.1 & 9.4 & 6.6 \\
\hline \multicolumn{7}{|l|}{ Exposure to household debt } \\
\hline Household debt to GDP & 0.0 & 0.0 & 0.0 & 1.5 & 1.5 & $\cdots$ \\
\hline
\end{tabular}

Source: Bank of Zambia

${ }^{1}$ Components do not add up to 100 because loans to households, government, and parastatals are included in loans classified by economic sectors.

${ }^{2}$ Liquid assets were redefined to exclude one-year Treasury bills beginning in 2005. 
Table 7. Zambia: Millennium Development Goals

\begin{tabular}{|c|c|c|c|c|c|c|}
\hline & 1990 & 1994 & 1997 & 2000 & 2003 & 2005 \\
\hline \multicolumn{7}{|l|}{ Goal 1: Eradicate extreme poverty and hunger ${ }^{1}$} \\
\hline Population below US\$ 1 dollar a day (in percent) & 64.6 & 73.6 & 66.0 & $\ldots$ & 76.0 & 64.0 \\
\hline Poverty gap ratio at US\$1 dollar a day (in percent) & 38.9 & 42.7 & 34.0 & $\ldots$ & 36.0 & 33.0 \\
\hline Percentage share of income or consumption held by poorest 20 percent & $\ldots$ & $\ldots$ & 3.3 & $\ldots$ & $\ldots$ & $\ldots$ \\
\hline Poverty headcount, national (percent of population) & $\ldots$ & $\ldots$ & 72.9 & $\ldots$ & $\ldots$ & 68.0 \\
\hline Prevalence of underweight children (percent of children under 5) & $\ldots$ & $\ldots$ & 23.5 & $\ldots$ & 23.0 & $\ldots$ \\
\hline Population below minimum level of dietary energy consumption (in percent) & $\ldots$ & $\ldots$ & 48.0 & $\ldots$ & 47.0 & 46.0 \\
\hline \multicolumn{7}{|l|}{ Goal 2: Achieve universal primary education ${ }^{2}$} \\
\hline Net primary enrollment ratio (percent of relevant age group) & 79.1 & $\ldots$ & 64.9 & 62.6 & 79.8 & 88.9 \\
\hline Primary completion rate, total (percent of relevant age group) & $\ldots$ & $\ldots$ & 61.6 & 56.3 & 66.2 & 77.5 \\
\hline Percentage of pupils starting grade 1 who reach grade 5 (in percent) & $\ldots$ & $\ldots$ & 78.2 & 98.5 & $\ldots$ & $\ldots$ \\
\hline Youth literacy rate (percent ages 15-24) & 81.2 & $\cdots$ & $\ldots$ & 69.4 & $\ldots$ & 69.5 \\
\hline \multicolumn{7}{|l|}{ Goal 3: Promote gender equality ${ }^{3}$} \\
\hline Ratio of girls to boys in primary and secondary education (in percent) & $\ldots$ & $\ldots$ & 89.7 & 90.4 & 92.3 & $\ldots$ \\
\hline Ratio of young literate females to males (percent ages 15-24) & 88.1 & $\ldots$ & $\ldots$ & $\ldots$ & $\ldots$ & 91.2 \\
\hline Share of women employed in the nonagricultural sector (in percent) & 29.4 & $\ldots$ & $\ldots$ & $\ldots$ & $\ldots$ & $\ldots$ \\
\hline Proportion of seats held by women in national parliament (in percent) & 7.0 & $\cdots$ & 10.0 & 10.0 & 12.0 & 12.7 \\
\hline \multicolumn{7}{|l|}{ Goal 4: Reduce child mortality ${ }^{4}$} \\
\hline Under 5 mortality rate (per 1,000) & 180.0 & 182.0 & $\ldots$ & 182.0 & 182.0 & 182.0 \\
\hline Infant mortality rate (per 1,000 live births) & 101.0 & 102.0 & $\ldots$ & 102.0 & 102.0 & 102.0 \\
\hline Immunization, measles (percent of children under 12 months) & 90.0 & 96.0 & 86.0 & 85.0 & 84.0 & 84.0 \\
\hline \multicolumn{7}{|l|}{ Goal 5: Improve maternal health ${ }^{5}$} \\
\hline Maternal mortality ratio (modeled estimate, per 100,000 live births) & $\ldots$ & $\ldots$ & $\ldots$ & 750.0 & $\ldots$ & $\ldots$ \\
\hline Births attended by skilled health staff (percent of total) & 50.5 & $\ldots$ & 46.5 & 47.1 & 43.4 & $\ldots$ \\
\hline \multicolumn{7}{|l|}{ Goal 6: Combat HIVIAIDS, malaria, and other diseases ${ }^{6}$} \\
\hline Prevalence of HIV, total (percent of population ages 15-49) & $\ldots$ & $\ldots$ & $\ldots$ & 16.7 & 16.9 & 17.0 \\
\hline Contraceptive prevalence rate (percent of women ages 15-49) & $\ldots$ & 48.1 & 25.9 & 53.5 & 34.0 & $\ldots$ \\
\hline Number of children orphaned by HIVIAIDS & $\ldots$ & $\ldots$ & $\ldots$ & 570,000 & 630,000 & $\ldots$ \\
\hline Incidence of tuberculosis (per 100,000 people) & 296.8 & 498.9 & 575.5 & 605.0 & 658.3 & 600.1 \\
\hline Tuberculosis cases detected under DOTS (in percent) & $\cdots$ & $\cdots$ & $\ldots$ & $\cdots$ & 62.0 & 51.6 \\
\hline \multicolumn{7}{|l|}{ Goal 7: Ensure environmental sustainability ${ }^{7}$} \\
\hline Forest area (percent of total land area) & 66.1 & $\ldots$ & $\ldots$ & 60.1 & $\ldots$ & 57.1 \\
\hline Nationally protected areas (percent of total land area) & $\ldots$ & $\ldots$ & $\ldots$ & $\ldots$ & 31.9 & $\ldots$ \\
\hline GDP per unit of energy use (PPP US\$ per kg oil equivalent) & 1.4 & 1.3 & 1.3 & 1.3 & 1.4 & 1.5 \\
\hline $\mathrm{CO} 2$ emissions (metric tons per capita) & 0.3 & 0.3 & 0.2 & 0.2 & 0.2 & $\ldots$ \\
\hline Access to an improved water source (percent of population) & 50.0 & $\ldots$ & $\ldots$ & $\ldots$ & 58.0 & $\ldots$ \\
\hline Access to improved sanitation (percent of population) & 44.0 & $\ldots$ & $\ldots$ & $\ldots$ & 55.0 & $\ldots$ \\
\hline Access to secure tenure (percent of population) & $\ldots$ & $\ldots$ & $\ldots$ & $\ldots$ & $\ldots$ & $\ldots$ \\
\hline \multicolumn{7}{|l|}{ Goal 8: Develop a Global Partnership for Development ${ }^{8}$} \\
\hline Youth unemployment rate (percent of total labor force ages 15-24) & 20.9 & $\ldots$ & $\ldots$ & $\ldots$ & $\ldots$ & $\ldots$ \\
\hline Fixed line and mobile telephones (per 1,000 people) & 7.8 & 8.6 & 8.2 & 17.0 & 29.2 & 89.2 \\
\hline Personal computers (per 1,000 people) & $\ldots$ & $\ldots$ & 5.8 & 6.5 & 8.4 & 9.8 \\
\hline
\end{tabular}

Source: World Development Indicators database, August 2007.

Note: In some cases the data are for earlier or later years than those stated.

${ }^{1}$ Goal 1 targets: Halve, between 1990 and 2015, the proportion of people whose income is less than one dollar a day. Halve, between 1990 and 2015, the proportion of people who suffer from hunger.

${ }^{2}$ Goal 2 target: Ensure that, by 2015, children everywhere, boys and girls alike, will be able to complete a full course of primary schooling.

${ }^{3}$ Goal 3 target: Eliminate gender disparity in primary and secondary education preferably by 2005 and to all levels of education no later than 2015.

${ }^{4}$ Goal 4 target: Reduce by two-thirds, between 1990 and 2015, the under-5 mortality rate.

${ }^{5}$ Goal 5 target: Reduce by three-quarters, between 1990 and 2015, the maternal mortality ratio.

${ }^{\circ}$ Goal 6 targets: Have halted by 2015, and begun to reverse, the spread of HIV/AIDS. Have halted by 2015 , and begun to reverse, the incidence of malaria and other major diseases.

${ }^{7}$ Goal 7 targets: Integrate the principles of sustainable development into country policies and programs and reverse the loss of environmental resources. Halve, by 2015 , the proportion of people without sustainable access to safe drinking water.

${ }^{\gamma}$ Goal 8 targets: Develop further an open, rule-based, predictable, nondiscriminatory trading and financial system. Address the special seeds of the least developed countries. Address the special needs of landlocked countries and small island developing states. 
Table 8. Zambia: Macroeconomic Framework, 2006-2012

\begin{tabular}{|c|c|c|c|c|c|c|c|}
\hline & 2006 & $\begin{array}{r}2007 \\
\text { Proj } \\
\end{array}$ & $\begin{array}{r}2008 \\
\text { Proj } \\
\end{array}$ & $\begin{array}{c}2009 \\
\text { Proj. }\end{array}$ & $\begin{array}{c}2010 \\
\text { Proj. }\end{array}$ & $\begin{array}{c}2011 \\
\text { Proj. }\end{array}$ & $\begin{array}{c}2012 \\
\text { Proj. }\end{array}$ \\
\hline National account and prices & \multicolumn{7}{|c|}{ (In percent changes) } \\
\hline GDP at constant prices & 6.2 & 6.2 & 6.5 & 6.5 & 6.0 & 6.0 & 6.0 \\
\hline \multicolumn{8}{|l|}{ Consumer prices (average) } \\
\hline Headline & 9.0 & 10.8 & 7.3 & 5.9 & 5.0 & 5.0 & 5.0 \\
\hline Underlying (excluding food) & 13.6 & 16.5 & 7.5 & 5.9 & 5.0 & 5.0 & 5.0 \\
\hline \multirow[t]{2}{*}{ Consumer prices (end of period) } & 8.2 & 9.0 & 7.0 & 5.0 & 5.0 & 5.0 & 5.0 \\
\hline & \multicolumn{7}{|c|}{ (In percent of GDP) } \\
\hline Gross investments & 22.6 & 24.1 & 22.7 & 22.1 & 22.5 & 23.3 & 23.7 \\
\hline Government & 3.9 & 6.2 & 6.1 & 6.9 & 7.2 & 7.2 & 7.2 \\
\hline Private & 18.7 & 17.9 & 16.6 & 15.2 & 15.3 & 16.1 & 16.5 \\
\hline National savings & 25.5 & 20.1 & 21.6 & 20.1 & 19.7 & 19.3 & 19.5 \\
\hline Gross foreign savings & -2.9 & 4.0 & 1.1 & 2.0 & 2.8 & 4.0 & 4.2 \\
\hline \multicolumn{8}{|l|}{ Central government budget ${ }^{1}$} \\
\hline Overall balance & 18.6 & -0.6 & -1.9 & -1.7 & -1.7 & -1.7 & -1.7 \\
\hline (excluding grants) & -7.4 & -5.4 & -6.5 & -5.9 & -5.7 & -5.6 & -5.3 \\
\hline Revenue & 16.9 & 18.4 & 18.4 & 18.7 & 19.0 & 19.1 & 19.2 \\
\hline Grants & 26.0 & 4.9 & 4.6 & 4.1 & 4.0 & 3.9 & 3.6 \\
\hline Total expenditure $^{2}$ & 24.3 & 23.8 & 24.8 & 24.5 & 24.7 & 24.7 & 24.5 \\
\hline Money and credit (end of period) & \multicolumn{7}{|c|}{ (In percent changes) } \\
\hline Broad money (M3) & 45.1 & 6.0 & 13.1 & 12.1 & 12.4 & 10.9 & 10.6 \\
\hline Domestic credit to the private sector & 54.3 & 35.0 & 21.0 & 20.0 & 20.0 & 20.0 & 22.0 \\
\hline External sector & \multicolumn{7}{|c|}{ (In percent of GDP) } \\
\hline Current account (including official grants) & 2.9 & -4.0 & -1.1 & -2.0 & -2.8 & -4.0 & -4.2 \\
\hline Current account (excluding official grants) & -1.3 & -8.8 & -6.1 & -6.8 & -7.8 & -8.8 & -8.7 \\
\hline \multicolumn{8}{|c|}{ (In percent of exports of goods and services) } \\
\hline NPV of external public debt (including IMF) & 16.0 & 13.7 & 14.3 & 17.0 & 20.1 & 22.2 & 22.8 \\
\hline \multicolumn{8}{|c|}{ (In percent changes) } \\
\hline Exports of goods and services & 66.0 & 12.0 & 3.7 & -7.0 & -7.2 & -0.6 & 4.3 \\
\hline Imports of goods and services & 22.4 & 31.5 & 0.6 & 1.5 & 0.8 & 3.9 & 5.1 \\
\hline Terms of trade (deterioration -) & 54.8 & 5.4 & -11.3 & -20.3 & -17.5 & -8.1 & -3.0 \\
\hline Copper export price (U.S. dollars per pound) & 82.3 & 7.6 & -11.8 & -25.0 & -22.2 & -10.0 & -3.3 \\
\hline \multicolumn{8}{|c|}{ (In millions of U.S. dollars, unless otherwise indicated) } \\
\hline Gross international reserves (in US\$ millions) & 595 & 850 & 1,009 & 1,167 & 1,326 & 1,355 & 1,399 \\
\hline In months of imports & 2.2 & 2.4 & 2.8 & 3.2 & 3.6 & 3.6 & 3.5 \\
\hline
\end{tabular}

Sources: Zambian authorities; and IMF staff estimates and projections.

${ }^{1}$ Grants in 2006 include MDRI debt cancellation amounting to 21.4 percent of GDP.

${ }^{2}$ Including discrepancy between the above-the-line balance and below-the-line financing. 


\section{Annex I. Joint IMF/World Bank Debt Sustainability Analysis 2007 under the Debt Sustainability Framework for Low Income Countries ${ }^{1}$}

External and public debt dynamics are assessed using the Low Income Country Debt Sustainability Analysis (LIC DSA) framework. ${ }^{2}$ Fund and Bank staffs have concluded that Zambia's risk of external debt distress is low. Total public debt is sustainable under all scenarios. Zambia's debt sustainability outlook has strengthened substantially since 2005, mainly because of higher copper export receipts and debt relief received under the HIPC and MDRI Initiatives. The baseline scenario shows that Zambia's public debt will improve further over the medium term as long as economic policies remain appropriate. Debt ratios are expected to continue to be manageable, well below the risk thresholds, through 2027.

\section{Underlying MACROECONOMic ASSUMPTIONS}

\section{The medium-term framework assumes that the Zambian economy will} continue to perform well. The baseline scenario assumes that real GDP growth remains strong, reflecting large-scale investments in mining and other sectors. The current account deficit is expected to widen over the long run as copper prices fall. Fiscal policies are assumed to remain targeted on limiting domestic borrowing to about one percent of GDP annually. The key assumptions are summarized below. The assumptions for this LIC DSA are broadly in line with those underlying the 2005 LIC DSA, except for GDP growth. The more positive copper sector developments in 2006 and 2007 have been incorporated. While stronger growth is assumed in the medium term, a more conservative approach is taken over the long term.

\section{The baseline scenario is based on the following assumptions:}

- Economic growth: Real GDP would continue to grow at 6-61/2 percent a year through 2012, supported primarily by developments in the copper and construction sectors. Over time, however, the Zambian economy is expected to diversify as noncopper sectors expand in response to an improved business environment and infrastructure. In the long run Zambia's growth is expected to stabilize at $4 \frac{1}{2}$ percent.

- Inflation: Inflation should decline to about 5 percent, if monetary and fiscal policies continue to be prudent.

\footnotetext{
1 Prepared by the IMF and World Bank staffs.

2 The previous LIC DSA (IMF Country Report No. 06/39) showed that Zambia was at a low risk of debt distress. At that time, Zambia had reached HIPC completion but relief under the MDRI has not yet been delivered.
} 
- External sector: Copper export volumes would grow at an average annual rate of about 10 percent through 2012 and then 41/2 percent through 2027. While copper will remain Zambia's most important export, its share in total exports is expected to fall from 78 percent in 2006 to 67 percent by 2012 and then to about 63 percent by $2027 .{ }^{3}$ Imports are assumed to grow in line with GDP in the long run. Annual foreign direct investment would peak at 7 percent of GDP in 2007 and decline to an average of $2 \frac{1}{2}$ percent for 2008-12 and to $1 \frac{1 / 2}{2}$ percent of GDP over the long run.

- Government revenue and expenditure: Government revenues would rise gradually from 18.4 percent of GDP in 2007 to 20 percent by 2027, mainly from improvements in tax administration; no change in the tax system is assumed. Expenditures stay constant at about 25 percent of GDP.

- $\quad$ Financing: Discussions with the authorities and main donors indicate that there are no plans currently to scale up foreign financing (grants and loans), except for one donor. The baseline scenario therefore assumes that annual external support will remain at about 6 percent of GDP during 2007-12, and then decline to $4 \frac{1}{2}$ percent of GDP during 2013-17 and to 3 percent of GDP by $2027 .{ }^{4}$ The baseline assumes support from emerging creditors, albeit at low levels. Annual external borrowing would remain at about 1 percent of GDP through 2012 and then decline to about $3 / 4$ percent through 2017 and $1 / 2$ percent for the rest of the period. Domestic financing would fall to 1 percent of GDP by 2009 and remain there over the long term. These funds would be used mainly to finance infrastructure projects.

- Contingent liabilities: Public Service Pension Fund's liabilities would grow by $1 / 2$ percent of GDP per year.

\section{EXternal DebT Sustainability Analysis}

\section{Background}

3. As a result of the HIPC and MDRI Initiatives, Zambia's stock of external debt has declined substantially. ${ }^{5}$ The external debt-to-GDP ratio declined from about 85 percent

\footnotetext{
${ }^{3}$ The nominal growth of exports in U.S. dollar terms is negative for 2009-11 because the price of copper is assumed to decline at an average annual rate of 20 percent over the next three years and at lower rates through 2013, in line with WEO projections. It is assumed that the price of copper will stabilize after 2013.

${ }^{4}$ In the baseline scenario a sizeable portion of grants are allocated to finance investment projects and classified as capital grants.

${ }^{5}$ Zambia reached the completion point under the Enhanced HIPC Initiative in April 2005. MDRI relief was delivered in January 2006.
} 
at the end of 2005 to about 9 percent at the end of 2006. Several creditors have already delivered their share of debt relief. ${ }^{6}$

- Multilateral Creditors: All multilateral creditors have provided HIPC relief. The AfDF, IDA, and the Fund have fully delivered their share of MDRI relief.

- $\quad$ Paris Club Creditors: Brazil, a creditor invited to participate in the Paris Club meeting in which relief beyond HIPC was agreed, has not yet delivered the relief expected, but is seeking approval from its Senate to do so. Only one full member of the Paris Club (Russia) has not granted beyond HIPC relief to Zambia, but discussions have started. ${ }^{7}$

- $\quad$ Non-Paris Club Creditors: Of the nine non-Paris Club creditors, China and India have provided relief on claims held by their governments but not on those held by public enterprises - Zambia plans to contact them. Two creditors, Bulgaria and Iraq, have not yet provided HIPC relief, but the authorities have contacted them. Three non-Paris Club creditors (Kuwait, Romania, and Yugoslavia) sold their claims on Zambia to private creditors and have been fully repaid. Debt to the other two nonParis Club creditors was fully repaid without any HIPC relief.

Zambia: Public External Debt

\begin{tabular}{crrrrr} 
& & & (In percent of GDP) & & (In percent of exports of goods and services) \\
\cline { 2 - 3 } & 2005 & 2006 & 2005 & 2006 \\
\hline Gross external debt & 86.0 & 8.8 & 251.9 & 23.3 \\
Medium- and long-term debt & 86.0 & 8.8 & 251.9 & 23.3 \\
Multilateral & 49.1 & 5.5 & 251.9 & 23.3 \\
IMF & 8.1 & 0.4 & 143.8 & 14.6 \\
Other & 41.0 & 5.1 & 23.8 & 1.0 \\
Bilateral official & 35.9 & 2.7 & 120.0 & 13.6 \\
Paris Club & 32.8 & 1.9 & 105.1 & 7.3 \\
Other & 3.0 & 0.9 & 96.2 & 5.0 \\
Suppliers and other & 1.0 & 0.6 & 3.0 & 1.5 \\
\hline
\end{tabular}

Sources: Bank of Zambia; and staff estimates.

\footnotetext{
${ }^{6}$ Arrears to private creditors, which accumulated because of limitations on the availability of foreign exchange prior to 1985 , have been excluded, since verification of outstanding claims to private individuals is not completed.

${ }^{7}$ Russia is working with the World Bank to develop a debt-for-development swap. This analysis assumes that Russia has already delivered debt relief beyond HIPC.
} 


\section{Results of the External DSA}

4. External debt burden indicators have improved substantially and are expected to remain well below the risk thresholds over the projection period in the baseline scenario (Table 1). Zambia's external debt ratios remain well below the risk thresholds. ${ }^{8}$ The stock of debt is expected to increase to about 12 percent of GDP through 2011 and then decline to single digits by 2017 . The NPV of debt-to-GDP is expected to climb from about 6 percent in 2007 to about 7 percent by 2011 and then decline to about 4 percent - below the 40 percent threshold. As copper prices decline and production slows, the NPV of debt-toexports is projected to increase from 14 percent in 2007 to about 23 percent in 2012 and stay there through 2027, well below the 150 percent threshold. The debt service-to-exports ratio is expected to increase from 1 percent in 2007 to 2 percent by 2012 and to 2.2 percent by 2027 .

\section{The standard sensitivity analysis points to a much reduced risk of debt distress}

(Table 2). Zambia's external debt position has become more resilient to exogenous shocks. In the alternative scenarios, the ratio that deteriorates most is the NPV of debt-to-exports. This occurs when key variables are assumed to grow at their historical averages (19972006), which reflect the poor performance of the Zambian economy from 1997 through 2002. The external current account deficit would remain at 10.7 percent of GDP from 2008 to 2027-significantly higher than the deficit assumed in the baseline scenario (3 percent by 2020). As a result, the NPV of debt-to-exports rises to 45 percent by 2017 and to 106 percent by 2027 . All bound tests result in higher but stable ratios well below the thresholds. Thus, even under difficult circumstances, Zambia's risk of debt distress is low. The scenario based on historical averages points to the need to diversify Zambia's exports away from copper as a means to make it even more resilient to external shocks.

\section{Plans for substantial investment in power generation have been under discussion} for a long time (Table 1). Therefore, the 2005 LIC DSA included an alternative scenario of nonconcessional foreign borrowing (US\$1 billion for 2008-11). ${ }^{9}$ The update assumes construction of three new hydropower plants (Itezhi Tezhi, Kariba North, and Lower Kafue Gorge) during 2009-15, the financing of which requires US\$1.2 billion. However, slightly more than 40 percent is expected to be financed with foreign direct investment, reducing the nonconcessional borrowing required to about US\$690 million. ${ }^{10}$ The non-concessional borrowing will not significantly change the external debt outlook. During the construction period, imports are expected to increase, but as projects are completed exports of electricity

\footnotetext{
${ }^{8}$ The World Bank's 2006 Country Policy and Institutions Assessment (CPIA) based on the three-year average ranks Zambia as a medium performer. Thus, the external debt burden thresholds for Zambia are (i) NPV of debt-to-GDP, 40 percent; (ii) NPV of debt-to-exports, 150 percent; (iii) NPV of debt-to-revenue, 250 percent; and (iv) debt service to exports, 20 percent, and to revenue, 30 percent.

${ }^{9}$ This scenario assumed an 8 percent interest rate, 5-year grace period, and 17-year maturity.

${ }^{10}$ This scenario assumes a 6 percent interest rate, 5-year grace period, and 15-year maturity.
} 
will come on stream. The NPV of debt-to-export ratio will peak at 37 percent in 2012 (23 percent in the baseline scenario) but will converge to the baseline scenario by 2027 . The assumed borrowing on nonconcessional terms does not pose any threat to the external debt sustainability; debt indicators even under the bound test are below the thresholds.

\section{Public Debt Sustainability Assessment}

\section{Background}

7. The size and composition of Zambia's public

Zambia: Structure of Public Sector Debt debt have significantly changed. At end 2005, total public debt was about 105 percent of GDP, 18 percent of which was domestic debt. After extensive debt relief from foreign creditors, Zambia's public debt decreased to 27 percent of GDP and domestic debt's share increased to about 70 percent.

\begin{tabular}{lrr}
\hline & 2005 & 2006 \\
\hline Public debt & 100.0 & 100.0 \\
External debt & 81.8 & 32.2 \\
Domestic debt & 18.2 & 67.8 \\
\hline
\end{tabular}

Sources: Bank of Zambia; and staff estimates.

8. As of end 2006, the domestic debt had two main components (i) government securities (15.9 percent of GDP), which are denominated in local currency only; and (ii) domestic arrears (2.6 percent of GDP). Most securities are marketable (13 percent of GDP), however, there is no significant secondary market. Foreign investment in government securities began in early 2005, after Zambia reached its HIPC completion point. The estimated stock of government securities in the hands of foreigners has increased from about US $\$ 150$ million at end-2005 to about US\$200 million by end-September 2007 (about 13 percent of the total domestic debt), of which close to 70 percent is short-term debt. This indicates that the rollover risk is low and the possible pressure on reserves of a change in sentiment of foreign investors is moderate.

\section{Results of the Public DSA}

9. In the baseline scenario, Zambia's public debt is expected to decline over the projection period (Table 3). The NPV of domestic debt would decline from 18.6 percent of GDP in 2006 to 10.4 percent by 2027. As a result of this and the decline in external debt, the NPV of public sector debt-to-GDP would drop from about 27 percent to 16 percent by 2027 . Thus, Zambia's public debt is considered manageable, as long as the authorities implement a cautious debt policy.

10. The standard sensitivity analysis shows that Zambia's public debt is also more resilient to shocks. The alternative scenarios and bound tests show that the outlook for public debt sustainability is benign, except in the case of permanently lower GDP. In this scenario the NPV of debt-to-revenue rises from about 97 percent to about 139 percent. In the 
bound tests, the NPV of debt-to-revenue ratio climbs to about 194 percent by 2027, which underscores the importance of sound macroeconomic policies to achieve higher GDP growth.

\section{Conclusions}

11. Application of the LIC-DSA framework indicates that Zambia's debt sustainability outlook has strengthened substantially. Fund and Bank staffs consider that Zambia is at low risk of external debt distress. The external debt indicators remain well below the thresholds in the alternative scenarios and the bound stress tests. The risks to public debt are also low. 
Table 1. Zambia: External Debt Sustainability Framework, Baseline Scenario, 2005-27 ${ }^{1}$

(In percent of GDP, unless otherwise indicated)

\begin{tabular}{|c|c|c|c|c|c|c|c|c|c|c|c|c|c|c|}
\hline & \multicolumn{2}{|c|}{ Actual } & \multirow{2}{*}{$\begin{array}{l}\text { Historical } \\
\text { Average 6/ }\end{array}$} & \multirow{2}{*}{$\begin{array}{l}\text { Standard } \\
\text { Deviation 6/ }\end{array}$} & \multicolumn{6}{|c|}{ Projections } & \multirow[b]{2}{*}{$\begin{array}{l}2007-12 \\
\text { Average }\end{array}$} & \multirow[b]{2}{*}{2017} & \multirow[b]{2}{*}{2027} & \multirow[b]{2}{*}{$\begin{array}{l}2013-27 \\
\text { Average } \\
\end{array}$} \\
\hline & 2005 & 2006 & & & 2007 & 2008 & 2009 & 2010 & 2011 & 2012 & & & & \\
\hline External debt (nominal) $^{1}$ & 86.0 & 8.8 & & & 9.9 & 10.1 & 10.7 & 11.4 & 11.7 & 11.5 & & 9.6 & 5.4 & \\
\hline $\mathrm{o} / \mathrm{w}$ public and publicly guaranteed (PPG) & 86.0 & 8.8 & & & 9.9 & 10.1 & 10.7 & 11.4 & 11.7 & 11.5 & & 9.6 & 5.4 & \\
\hline Change in external debt & -40.7 & -77.2 & & & 1.1 & 0.2 & 0.6 & 0.7 & 0.3 & -0.2 & & -0.4 & -0.4 & \\
\hline Identified net debt-creating flows & -26.3 & -32.9 & & & -0.3 & -0.1 & 2.6 & 4.1 & 5.0 & 5.1 & & 1.5 & 2.1 & \\
\hline Non-interest current account deficit & 9.4 & -0.2 & 11.1 & 4.8 & 7.4 & 4.9 & 5.7 & 6.8 & 7.6 & 7.7 & & 3.6 & 3.7 & 3.9 \\
\hline Deficit in balance of goods and services & 2.5 & -8.0 & & & -3.1 & -4.1 & -0.7 & 2.2 & 3.1 & 3.2 & & 0.3 & 2.0 & \\
\hline Exports & 33.7 & 37.5 & & & 40.7 & 40.0 & 36.1 & 33.0 & 31.4 & 30.4 & & 28.0 & 17.4 & \\
\hline Imports & 36.2 & 29.6 & & & 37.6 & 35.9 & 35.4 & 35.1 & 34.4 & 33.6 & & 28.3 & 19.4 & \\
\hline Net current transfers (negative $=$ inflow) & -3.6 & -2.4 & -4.6 & 1.2 & -2.9 & -2.9 & -2.5 & -2.5 & -1.7 & -1.4 & & -1.1 & -0.8 & -1.0 \\
\hline $\mathrm{o} / \mathrm{w}$ official & -3.9 & -3.1 & & & -3.6 & -3.7 & -3.4 & -3.6 & -2.8 & -2.5 & & -1.9 & -1.2 & \\
\hline Other current account flows (negative $=$ net inflow) & 10.5 & 10.2 & & & 13.4 & 11.9 & 8.9 & 7.1 & 6.2 & 5.9 & & 4.4 & 2.5 & \\
\hline Net FDI (negative = inflow) & -5.2 & -4.3 & -5.5 & 1.9 & -7.2 & -4.5 & -2.5 & -2.1 & -2.1 & -2.1 & & -1.7 & -1.5 & -1.6 \\
\hline Endogenous debt dynamics $^{2}$ & -30.4 & -28.4 & & & -0.5 & -0.5 & -0.5 & -0.5 & -0.5 & -0.5 & & -0.3 & -0.2 & \\
\hline Contribution from nominal interest rate & 1.5 & 0.2 & & & 0.1 & 0.1 & 0.1 & 0.1 & 0.1 & 0.1 & & 0.2 & 0.1 & \\
\hline Contribution from real GDP growth & -5.0 & -3.6 & & & -0.5 & -0.6 & -0.6 & -0.6 & -0.7 & -0.6 & & -0.5 & -0.2 & \\
\hline Contribution from price and exchange rate changes & -27.0 & -25.0 & & & $\ldots$ & $\ldots$ & $\ldots$ & $\ldots$ & $\ldots$ & $\ldots$ & & $\ldots$ & $\ldots$ & \\
\hline Residual (3-4) ${ }^{3}$ & -14.5 & -44.3 & & & 1.4 & 0.3 & -2.1 & -3.4 & -4.7 & -5.3 & & -1.9 & -2.4 & \\
\hline $\mathrm{o} / \mathrm{w}$ exceptional financing & -6.6 & 0.0 & & & 0.0 & 0.0 & 0.0 & 0.0 & 0.0 & 0.0 & & 0.0 & 0.0 & \\
\hline NPV of external debt ${ }^{4}$ & & 6.0 & & & 5.6 & 5.7 & 6.1 & 6.6 & 7.0 & 6.9 & & 6.4 & 3.9 & \\
\hline In percent of exports & $\ldots$ & 16.0 & & & 13.7 & 14.3 & 17.0 & 20.1 & 22.2 & 22.8 & & 22.7 & 22.5 & \\
\hline NPV of PPG external debt & ... & 6.0 & & & 5.6 & 5.7 & 6.1 & 6.6 & 7.0 & 6.9 & & 6.4 & 3.9 & \\
\hline In percent of exports & ... & 16.0 & & & 13.7 & 14.3 & 17.0 & 20.1 & 22.2 & 22.8 & & 22.7 & 22.5 & \\
\hline In percent of government revenues & ... & 35.6 & & & 31.5 & 32.6 & 35.1 & 37.0 & 38.0 & 37.6 & & 34.3 & 21.0 & \\
\hline Debt service-to-exports ratio (in percent) & 97.1 & 60.4 & & & 1.3 & 0.9 & 0.9 & 1.0 & 1.4 & 1.9 & & 1.6 & 2.2 & \\
\hline PPG debt service-to-exports ratio (in percent) & 97.1 & 60.4 & & & 1.3 & 0.9 & 0.9 & 1.0 & 1.4 & 1.9 & & 1.6 & 2.2 & \\
\hline PPG debt service-to-revenue ratio (in percent) & 188.3 & 134.3 & & & 3.0 & 2.1 & 1.9 & 1.8 & 2.4 & 3.1 & & 2.4 & 2.0 & \\
\hline Total gross financing need (billions of U.S. dollars) & 2682.9 & 1981.7 & & & -225.4 & -88.0 & 234.8 & 444.8 & 748.7 & 1035.0 & & 373.4 & 1112.1 & \\
\hline Non-interest current account deficit that stabilizes debt ratio & 50.1 & 77.0 & & & 6.3 & 4.7 & 5.2 & 6.1 & 7.3 & 7.9 & & 3.9 & 4.0 & \\
\hline \multicolumn{15}{|l|}{ Key macroeconomic assumptions } \\
\hline Real GDP growth (in percent) & 5.2 & 6.2 & 3.7 & 2.3 & 6.2 & 6.5 & 6.5 & 6.0 & 6.0 & 6.0 & 6.2 & 5.0 & 4.5 & 4.7 \\
\hline GDP deflator in US dollar terms (change in percent) & 27.0 & 41.0 & 9.9 & 16.6 & -2.7 & -0.9 & -3.6 & -4.2 & -1.5 & 1.6 & -1.9 & 3.6 & 4.1 & 3.9 \\
\hline Effective interest rate (percent) ${ }^{5}$ & 1.6 & 0.3 & 2.0 & & 0.7 & 1.2 & 1.0 & 0.8 & 1.1 & 1.1 & 1.0 & 1.7 & 1.6 & 1.6 \\
\hline Growth of exports of G\&S (US dollar terms, in percent) & 19.6 & 66.8 & 17.3 & 29.5 & 12.0 & 3.7 & -7.1 & -7.3 & -0.7 & 4.3 & 0.8 & 6.3 & 2.8 & 4.8 \\
\hline Growth of imports of G\&S (US dollar terms, in percent) & 13.5 & 22.4 & 9.5 & 13.9 & 31.5 & 0.6 & 1.5 & 0.8 & 2.3 & 5.1 & 7.0 & 5.5 & 4.9 & 4.9 \\
\hline Grant element of new public sector borrowing (in percent) & $\ldots$ & $\ldots$ & $\ldots$ & $\ldots$ & 34.6 & 37.8 & 34.8 & 36.1 & 33.1 & 33.2 & 34.9 & 31.8 & 29.2 & 30.8 \\
\hline Aid flows (in billions of US dollars) ${ }^{7}$ & 1,075 & 572 & & & 756 & 709 & 718 & 757 & 681 & 683 & & 820 & 1,339 & \\
\hline $\mathrm{o} / \mathrm{w}$ Grants & 0.4 & 0.5 & & & 0.5 & 0.6 & 0.6 & 0.6 & 0.5 & 0.5 & & 0.7 & 1.2 & \\
\hline $\mathrm{o} / \mathrm{w}$ Concessional loans & 176.4 & 115.7 & & & 207.5 & 115.8 & 130.3 & 138.2 & 143.9 & 151.9 & & 155.0 & 184.5 & \\
\hline Grant-equivalent financing (in percent of GDP) $^{8}$ & $\ldots$ & $\ldots$ & & & 0.6 & 0.4 & 0.4 & 0.4 & 0.4 & 0.4 & & 0.2 & 0.1 & 0.2 \\
\hline Grant-equivalent financing (in percent of external financing) $8 /$ & $\ldots$ & $\ldots$ & & & 34.8 & 38.1 & 35.1 & 36.4 & 33.4 & 33.4 & & 32.1 & 29.6 & 31.2 \\
\hline \multicolumn{15}{|l|}{ Memorandum items: } \\
\hline Nominal GDP (billions of US dollars) & 7,271 & 10,893 & & & 11,261 & 11,884 & 12,212 & 12,408 & 12,956 & 13,949 & & 21,263 & 49,409 & \\
\hline (NPVt-NPVt-1)/GDPt-1 (in percent) & & & & & -0.2 & 0.5 & 0.6 & 0.6 & 0.6 & 0.5 & 0.4 & 0.4 & 0.1 & 0.3 \\
\hline
\end{tabular}

Source: Staff simulations.

${ }^{1}$ Includes both public and private sector external debt.

${ }^{2}$ Derived as $[\mathrm{r}-\mathrm{g}-\rho(1+\mathrm{g})](1+\mathrm{g}+\rho+\mathrm{g} \rho)$ times previous period debt ratio, with $\mathrm{r}=$ nominal interest rate; $\mathrm{g}=$ real GDP growth rate, and $\rho=$ growth rate of GDP deflator in U.S. dollar terms.

${ }^{3}$ Includes exceptional financing (i.e., changes in arrears and debt relief); changes in gross foreign assets; and valuation adjustments. It also includes grants allocated to finance investment projects, which are classified as

capital inflows in the balance of payments. For projections also includes contribution from price and exchange rate changes.

${ }^{4}$ Assumes that NPV of private sector debt is equivalent to its face value.

${ }^{5}$ Current-year interest payments divided by previous period debt stock.

${ }^{6}$ Historical averages and standard deviations are generally derived over the past 10 years, subject to data availability.

'Defined as grants, concessional loans, and debt relief.

${ }^{8}$ Grant-equivalent financing includes grants provided directly to the government and through new borrowing (difference between the face value and the NPV of new debt).

CInternational Monetary Fund. Not for Redistribution 
Table 2. Zambia: Sensitivity Analyses for Key Indicators of Public and Publicly Guaranteed External Debt, 2007-27 (In percent)

\begin{tabular}{|c|c|c|c|c|c|c|c|c|}
\hline & \multicolumn{8}{|c|}{ Projections } \\
\hline & 2007 & 2008 & 2009 & 2010 & 2011 & 2012 & 2017 & 2027 \\
\hline \multicolumn{9}{|c|}{ NPV of debt-to-GDP ratio } \\
\hline Baseline & 6 & 6 & 6 & 7 & 7 & 7 & 6 & 4 \\
\hline \multicolumn{9}{|l|}{ A. Alternative Scenarios } \\
\hline A1. Key variables at their historical averages in $2008-27^{1}$ & 6 & 9 & 10 & 10 & 9 & 8 & 13 & 18 \\
\hline A2. New public sector loans on less favorable terms in $2008-27^{2}$ & 6 & 6 & 7 & 7 & 8 & 8 & 8 & 6 \\
\hline A3. Non-concessional borrowing for power generation plants & 6 & 6 & 7 & 9 & 10 & 11 & 9 & 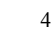 \\
\hline \multicolumn{9}{|l|}{ B. Bound Tests } \\
\hline B1. Real GDP growth at historical average minus one standard deviation in 2008-09 & 6 & 6 & 7 & 7 & 8 & 8 & 7 & $T$ \\
\hline B2. Export value growth at historical average minus one standard deviation in $2008-09^{3}$ & 6 & 10 & 15 & 16 & 17 & 16 & 12 & 5 \\
\hline B3. US dollar GDP deflator at historical average minus one standard deviation in 2008-09 & 6 & 6 & 7 & 7 & 8 & 8 & 7 &  \\
\hline B4. Net non-debt creating flows at historical average minus one standard deviation in $2008-09^{4}$ & 6 & 6 & 5 & 5 & 6 & 6 & 6 & 4 \\
\hline B5. Combination of B1-B4 using one-half standard deviation shocks & 6 & 5 & 2 & 3 & 3 & 3 & 4 & 3 \\
\hline B6. One-time 30 percent nominal depreciation relative to the baseline in $2008^{5}$ & 6 & 8 & 9 & 10 & 10 & 10 & 9 & 6 \\
\hline \multicolumn{9}{|c|}{ NPV of debt-to-exports ratio } \\
\hline Baseline & 14 & 14 & 17 & 20 & 22 & 23 & 23 & 22 \\
\hline \multicolumn{9}{|l|}{ A. Alternative Scenarios } \\
\hline A1. Key variables at their historical averages in $2007-26^{1}$ & 14 & 22 & 27 & 29 & 28 & 26 & 45 & 106 \\
\hline A2. New public sector loans on less favorable terms in $2007-262 /$ & 14 & 15 & 18 & 22 & 25 & 27 & 29 & 35 \\
\hline A3. Non-concessional borrowing for power generation plants & 14 & 14 & 19 & 27 & 33 & 37 & 32 & 22 \\
\hline \multicolumn{9}{|l|}{ B. Bound Tests } \\
\hline B1. Real GDP growth at historical average minus one standard deviation in 2008-09 & 14 & 14 & 17 & 20 & 22 & 23 & 23 & 22 \\
\hline B2. Export value growth at historical average minus one standard deviation in $2008-09^{3}$ & 14 & 30 & 54 & 62 & 66 & 67 & 55 & 39 \\
\hline B3. US dollar GDP deflator at historical average minus one standard deviation in 2008-09 & 14 & 14 & 17 & 20 & 22 & 23 & 23 & 22 \\
\hline B4. Net non-debt creating flows at historical average minus one standard deviation in 2008-09 ${ }^{4}$ & 14 & 15 & 14 & 17 & 19 & 19 & 20 & 21 \\
\hline B5. Combination of B1-B4 using one-half standard deviation shocks & 14 & 13 & 6 & 8 & 9 & 10 & 13 & 17 \\
\hline B6. One-time 30 percent nominal depreciation relative to the baseline in $2008^{5}$ & 14 & 14 & 17 & 20 & 22 & 23 & 23 & 22 \\
\hline
\end{tabular}

\section{Debt service-to-exports ratio}

\section{Baseline}

\section{A. Alternative Scenarios}

A1. Key variables at their historical averages in $2008-27^{1}$

A2. New public sector loans on less favorable terms in $2008-27^{2}$

A3. Non-concessional borrowing for power generation plants

B. Bound Tests

B1. Real GDP growth at historical average minus one standard deviation in 2008-09 B2. Export value growth at historical average minus one standard deviation in 2008-09 ${ }^{3}$

B3. US dollar GDP deflator at historical average minus one standard deviation in 2008-09

B4. Net non-debt creating flows at historical average minus one standard deviation in 2008-09 ${ }^{4}$

B5. Combination of B1-B4 using one-half standard deviation shocks

B6. One-time 30 percent nominal depreciation relative to the baseline in $2008^{5}$

Memorandum item:

Grant element assumed on residual financing (i.e., financing required above baseline) ${ }^{6}$

$\begin{array}{llllllll}1 & 1 & 1 & 1 & 1 & 2 & \mathbf{2} & 2 \\ 1 & 1 & 1 & 1 & 1 & 2 & \mathbf{2} & 6 \\ 1 & 1 & 1 & 1 & 2 & 2 & \mathbf{2} & 3 \\ 1 & 1 & 1 & 1 & 2 & 3 & \mathbf{3} & 2 \\ & & & & & & & \\ 1 & 1 & 1 & 1 & 1 & 2 & \mathbf{1} & 2 \\ 1 & 1 & 2 & 3 & 3 & 3 & \mathbf{4} & 4 \\ 1 & 1 & 1 & 1 & 1 & 2 & \mathbf{1} & 2 \\ 1 & 1 & 1 & 1 & 1 & 2 & \mathbf{1} & 2 \\ 1 & 1 & 1 & 1 & 1 & 1 & \mathbf{1} & 1 \\ & 1 & 1 & 1 & 1 & 2 & \mathbf{1} & 2 \\ 30 & 30 & 30 & 30 & 30 & 30 & \mathbf{3 0} & 30\end{array}$

Source: Staff projections and simulations.

${ }^{1}$ Variables include real GDP growth, growth of GDP deflator (in U.S. dollar terms), non-interest current account in percent of GDP, and non-debt creating flows.

${ }^{2}$ Assumes that the interest rate on new borrowing is by 2 percentage points higher than in the baseline., while grace and maturity periods are the same as in the baseline.

${ }^{3}$ Exports values are assumed to remain permanently at the lower level, but the current account as a share of GDP is assumed to return to its baseline level after the shock (implicitly : an offsetting adjustment in import levels).

${ }^{4}$ Includes official and private transfers and FDI.

${ }^{5}$ Depreciation is defined as percentage decline in dollar/local currency rate, such that it never exceeds 100 percent.

${ }^{6}$ Applies to all stress scenarios except for A2 (less favorable financing) in which the terms on all new financing are as specified in footnote 2.

\section{CInternational Monetary Fund. Not for Redistribution}


Figure 1. Zambia: Indicators of Public and Publicly Guaranteed External Debt Under Alternative Scenarios, 2007-2027
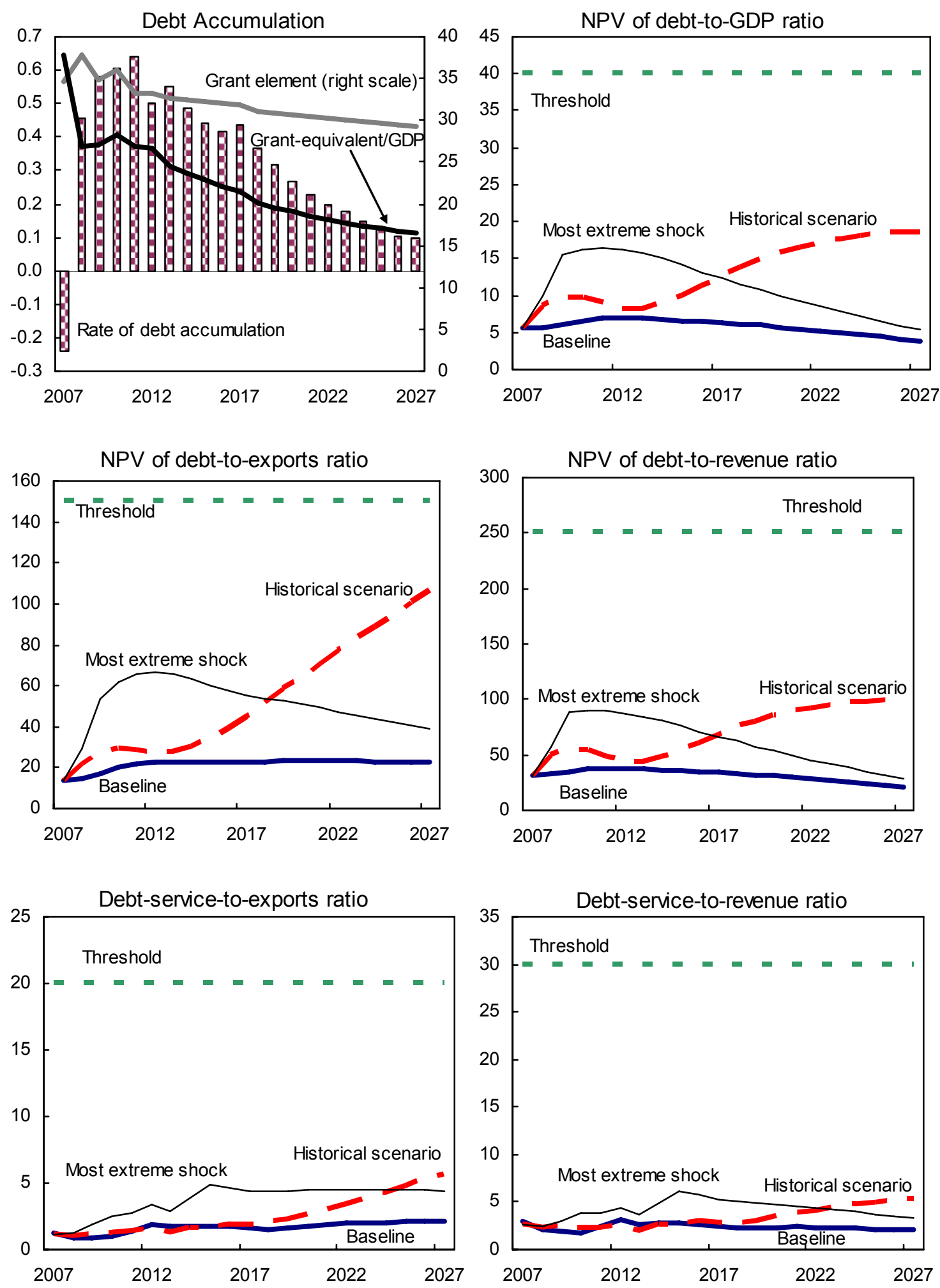

Source: Staff projections and simulations. 
Table 3.Zambia: Public Sector Debt Sustainability Framework, Baseline Scenario, 2005-27

(In percent of GDP, unless otherwise indicated)

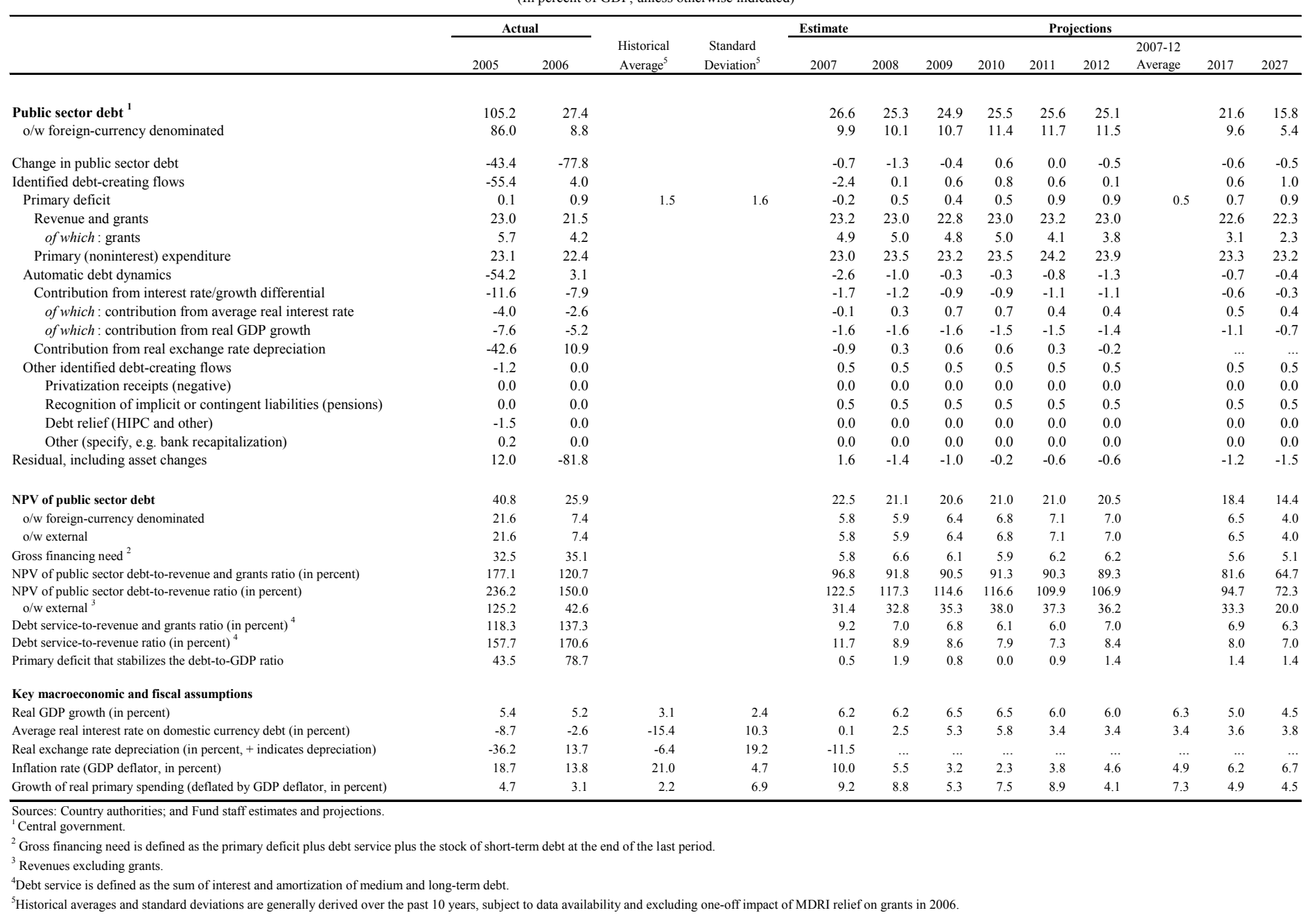


Table 4. Zambia: Sensitivity Analysis for Key Indicators of Public Debt 2007-27

\begin{tabular}{|c|c|c|c|c|c|c|c|c|}
\hline & & & & Project & ions & & & \\
\hline & 2007 & 2008 & 2009 & 2010 & 2011 & 2012 & 2017 & 2027 \\
\hline NPV of Debt-to-GD & & & & & & & & \\
\hline Baseline & 22 & 21 & 21 & 21 & 21 & 21 & 18 & 14 \\
\hline A. Alternative scenarios & & & & & & & & \\
\hline A1. Real GDP growth and primary balance are at historical averages & 22 & 23 & 24 & 26 & 27 & 28 & 30 & 29 \\
\hline A2. Primary balance is unchanged from 2006 & 22 & 21 & 22 & 22 & 22 & 22 & 21 & 17 \\
\hline A3. Permanently lower GDP growth ${ }^{1}$ & 22 & 21 & 21 & 22 & 23 & 22 & 24 & 31 \\
\hline B. Bound tests & & & & & & & & \\
\hline B1. Real GDP growth is at historical average minus one standard deviations in 2008-2009 & 22 & 23 & 26 & 28 & 30 & 32 & 37 & 44 \\
\hline B2. Primary balance is at historical average minus one standard deviations in 2008-2009 & 22 & 24 & 26 & 26 & 26 & 25 & 22 & 16 \\
\hline B3. Combination of B1-B2 using one half standard deviation shocks & 22 & 24 & 26 & 26 & 26 & 25 & 20 & 12 \\
\hline B4. One-time 30 percent real depreciation in 2008 & 22 & 24 & 23 & 24 & 23 & 23 & 20 & 15 \\
\hline B5. 10 percent of GDP increase in other debt-creating flows in 2008 & 22 & 30 & 30 & 30 & 30 & 30 & 26 & 20 \\
\hline NPV of Debt-to-Reven & & & & & & & & \\
\hline Baseline & 97 & 92 & 90 & 91 & 90 & 89 & 82 & 65 \\
\hline A. Alternative scenarios & & & & & & & & \\
\hline A1. Real GDP growth and primary balance are at historical averages & 97 & 98 & 103 & 111 & 114 & 118 & 128 & 125 \\
\hline A2. Primary balance is unchanged from 2006 & 97 & 93 & 94 & 97 & 96 & 95 & 91 & 75 \\
\hline A3. Permanently lower GDP growth ' & 97 & 93 & 93 & 96 & 97 & 97 & 104 & 139 \\
\hline B. Bound tests & & & & & & & & \\
\hline B1. Real GDP growth is at historical average minus one standard deviations in 2008-2009 & 97 & 100 & 112 & 121 & 128 & 135 & 161 & 194 \\
\hline B2. Primary balance is at historical average minus one standard deviations in 2008-2009 & 97 & 102 & 113 & 114 & 112 & 110 & 97 & 71 \\
\hline B3. Combination of B1-B2 using one half standard deviation shocks & 97 & 102 & 112 & 111 & 108 & 105 & 87 & 54 \\
\hline B4. One-time 30 percent real depreciation in 2008 & 97 & 103 & 102 & 103 & 101 & 99 & 88 & 67 \\
\hline B5. 10 percent of GDP increase in other debt-creating flows in 2008 & 97 & 132 & 131 & 132 & 131 & 129 & 116 & 88 \\
\hline
\end{tabular}

Debt Service-to-Revenue Ratio ${ }^{2}$

Baseline

\section{A. Alternative scenarios}

A1. Real GDP growth and primary balance are at historical averages

A2. Primary balance is unchanged from 2006

A3. Permanently lower GDP growth ${ }^{1}$

$\begin{array}{rrrrrrrr}9 & 8 & 9 & 9 & 9 & 10 & 11 & 13 \\ 9 & 8 & 8 & 7 & 7 & 8 & 8 & 8 \\ 9 & 8 & 8 & 7 & 7 & 8 & 10 & 15\end{array}$

\section{B. Bound tests}

B1. Real GDP growth is at historical average minus one standard deviations in 2008-2009

B2. Primary balance is at historical average minus one standard deviations in 2008-2009

B3. Combination of B1-B2 using one half standard deviation shocks

B4. One-time 30 percent real depreciation in 2008

B5. 10 percent of GDP increase in other debt-creating flows in 2008

$\begin{array}{rrrrrrrr}9 & 9 & 9 & 11 & 11 & 13 & 15 & 22 \\ 9 & 8 & 11 & 12 & 9 & 9 & 8 & 8 \\ 9 & 8 & 10 & 11 & 8 & 8 & 7 & 5 \\ 9 & 8 & 8 & 7 & 7 & 8 & 7 & 6 \\ 9 & 8 & 21 & 12 & 9 & 10 & 9 & 10\end{array}$

Sources: Country authorities; and Fund staff estimates and projections.

${ }^{1}$ Assumes that real GDP growth is at baseline minus one standard deviation divided by the square root of 20 (i.e., the length of the projection period).

${ }^{2}$ Revenues are defined inclusive of grants. 
Figure 2. Zambia: Indicators of Public Debt under Alternative Scenarios, 2007-27 ${ }^{1}$
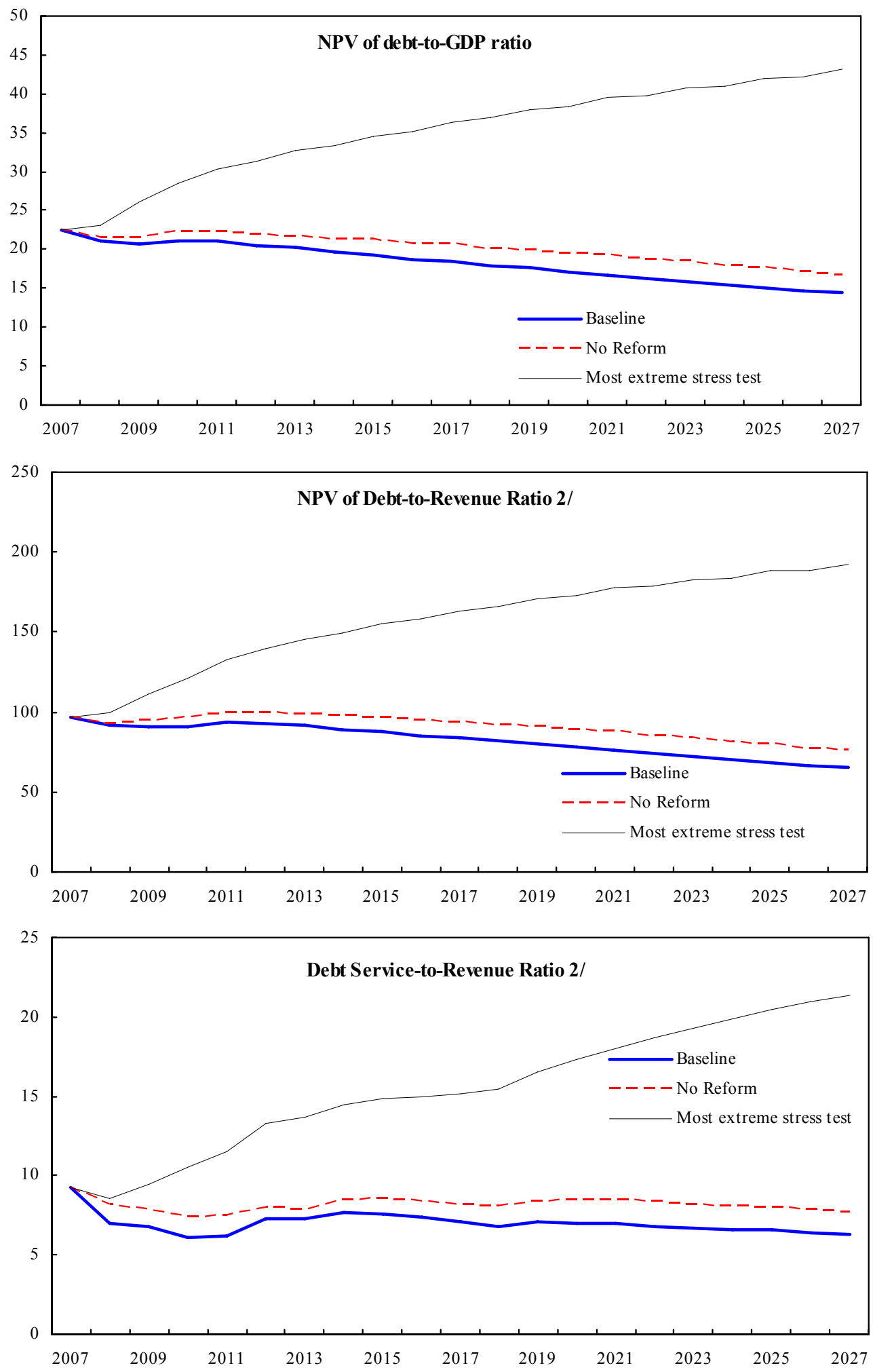

Source: Staff projections and simulations.

1/ Most extreme stress test is test that yields highest ratio in 2017.

2/ Revenue including grants. 
ANNEX II. ZAMbia: Relations With the Fund

(As of September 30, 2007)

I. Membership Status: Joined: September 23, 1965; Article VIII

II. General Resources Account:

SDR Million

Percent

Quota

Quota

489.10

100.0

489.10

100.0

0.02

Reserve position in Fund

0.0

III. SDR Department:

SDR Million

Percent

Allocation

Net cumulative allocation

68.30

100.0

Holdings

6.91

10.12

IV. Outstanding Purchases and Loans:

SDR Million

Percent

Quota

PRGF arrangements

55.02

V. Latest Financial Arrangements:

$\begin{array}{ccccc}\text { Type } & \text { Approval } & \text { Expiration } & \text { Amount Approved } & \text { Amount Drawn } \\ \text { Date } & \text { Date } & \text { (SDR Million) } & \text { (SDR Million) }\end{array}$

$\begin{array}{lllll}\text { PRGF } & 06 / 16 / 2004 & 09 / 30 / 2007 & 220.10 & 220.10 \\ \text { PRGF } & 03 / 25 / 1999 & 03 / 28 / 2003 & 278.90 & 237.52 \\ \text { PRGF } & 12 / 06 / 1995 & 12 / 05 / 1998 & 701.68 & 661.68\end{array}$

VI. Projected Payments to the Fund

(SDR million; based on existing use of resources and present holdings of SDRs)

\begin{tabular}{|c|c|c|c|c|c|}
\hline & & & comin & & \\
\hline & 2007 & 2008 & 2009 & 2010 & 2011 \\
\hline Principal & . & & $\ldots$ & 0.55 & 3.30 \\
\hline Charges/interest & $\underline{0.74}$ & 2.61 & 2.60 & 2.60 & 2.60 \\
\hline Total & 0.74 & 2.61 & 2.60 & 3.15 & 5.92 \\
\hline
\end{tabular}




\section{Implementation of HIPC Initiative}

\section{Enhanced \\ Framework}

Commitment of HIPC assistance

Decision point date

Dec. 2000

Assistance committed (1999 NPV terms) ${ }^{1}$

by all creditors (US\$ million)

Of which: Fund assistance (US\$ million)

(SDR equivalent in million)

468.80

Completion point date

April 2005

Disbursement of IMF assistance (SDR million)

Assistance disbursed to Zambia

Interim assistance

Completion point balance

117.20

Additional disbursements of interest income ${ }^{2}$

Total disbursements

508.27

\section{Implementation of MDRI Assistance}

I. Total debt relief (SDR million) ${ }^{3}$

Of which: MDRI

HIPC

II. Debt relief by facility (SDR million)

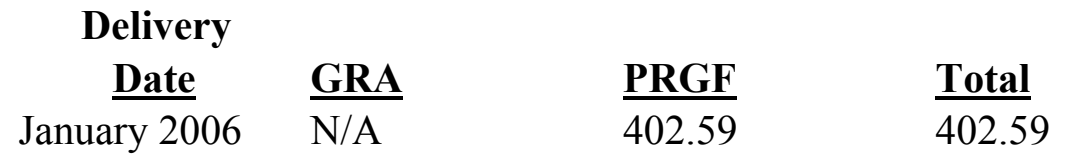

\footnotetext{
${ }^{1}$ Net present value (NPV) terms at the decision point under the enhanced framework.

${ }^{2}$ Under the enhanced framework, an additional disbursement is made at the completion point corresponding to interest income earned on the amount committed at the decision point but not disbursed during the interim period.

${ }^{3}$ The Multilateral Debt Relief Initiative (MDRI) provides 100 percent debt relief to eligible member countries that are qualified for assistance. The debt relief covers all debt owed to the Fund as of end-2004 that is outstanding at the time the member qualifies for the relief.
} 


\section{Safeguards Assessment}

Under the Fund's safeguards assessment policy, the Bank of Zambia (BoZ) is subject to an assessment for the PRGF arrangement, which was approved on June 16, 2004 and is scheduled to expire on June 15, 2007. A safeguards assessment of the BoZ was completed on October 20, 2004.

The authorities have taken the following steps in response to the recommendations made to address the weaknesses identified:

(i) In December 2004, the BoZ's board adopted the International Financial Reporting Standards as the basis for financial reporting.

(ii) In December 2004, the BoZ's board adopted a policy for the appointment of external auditors (three year-term appointment, renewable only once).

(iii) Pending the approval of an amendment to the Bank of Zambia Act, the BoZ and the Ministry of Finance and National Planning (MoFNP) have adopted a Memorandum of Understanding to exclude unrealized gains from profits available for distribution to government.

(iv) The Finance Department of the BoZ and the Economics Department of the BoZ have established a data monitoring committee to jointly reconcile the data reported to the Fund.

(v) Proposed amendments to the BoZ Act to strengthen the operational independence of the BoZ have been submitted for consideration as part of the current review of the country's constitution.

\section{Exchange Rate Arrangement}

The currency of Zambia is the kwacha. The exchange rate regime is a managed float with no predetermined path for the exchange rate. The official exchange rate is determined in the interbank market; the Bank of Zambia's (BoZ) buying rate is the simple average of the primary dealers' low bid rates, and the BoZ's selling rate is the simple average of the primary dealers' high offer rates. On April 19, 2002, Zambia accepted the obligations of Article VIII, Section 2, 3, and 4 of the Articles of Agreement. However, the Fund urged the authorities to eliminate the exchange restriction evidenced by the accumulation of external payments arrears, which is subject to Fund approval under Article VIII, as soon as possible. 


\section{Article IV Consultations}

Zambia is on the standard 12-month Article IV consultation cycle. The Executive Board concluded the last Article IV consultation on January 11, 2006.

\section{FSAP Participation and ROSC}

Zambia has participated in the financial sector assessment program (FSAP); an FSAP mission from the Fund and the World Bank conducted a comprehensive external assessment of the financial system April 30-May 15 and July 15-26, 2002.

The fiscal transparency module of a Report on Observance of Standards and Codes (ROSC) assessing compliance with the IMF's Code of Good Practices on Fiscal TransparencyDeclaration of Principles was issued to the Executive Board on October 31, 2001. A ROSCdata module was issued to the Executive Board on January 18, 2005.

\section{Technical Assistance (since 2003)}

\section{Resident advisors}

\section{Department}

FAD

2002-2003

\section{Position}

Advisor on public expenditure management

\section{Technical assistance missions}

\section{Department}

MFD

MCM

\section{Dates}

January and May 2003

September 2003

April-October 2004

April-May 2005

March 2006

September 2007

\section{Purpose}

Development of foreign exchange interbank market, monetary operations, and reform of the financial system Monetary operations, reform of the financial system, government securities market, and payments system Resolution of nonbank financial institutions (three missions) Liquidity management operations Liquidity management, monetary and exchange rate policies Institutional arrangements and operation of the foreign exchange market. 
STA

June 2004

Data ROSC

April-May 2005

Monetary Statistics (GDDS)

May 2005

February 2006

Government Finance Statistics (GDDS)

July 2006

Real Sector (GDDS)

Real Sector (GDDS)

FAD

July-August 2005

Regulations for the Public Finance Act

November 2005

Fiscal regime for copper mining

July 2006

Revenue administration

August 2006

Follow up on fiscal arrangements for the mining sector

October 2006

Review of tax policy

LEG

August 2004 -

February 2005

Assistance on amending legislation on nonbank financial institutions

May 2005

Strengthening the regulatory framework for

nonbank financial institutions

\section{Resident Representative}

A Fund Resident Representative first took up the position in Lusaka in June 1990. Mr. Birgir Arnason has been the Resident Representative since November 2006. 


\section{ANNEX III. ZAMbIA: RElations WITH THE WORLD BANK GROUP}

\section{A. Partnership for Development}

1. World Bank support to Zambia is based upon the country's PRSP, which is prepared through a participatory process led by the government. Zambia's Fifth National Development Plan 2006-10 (FNDP) serves as the country's revised PRSP and has been officially launched by the President in early 2007. In preparing the FNDP, the authorities held discussions with local leaders in all of Zambia's 72 districts. Sectoral Advisory Groups (SAGs), chaired by government officials with stakeholder representation, were then tasked to draft the comprehensive document, which underwent a review by stakeholders during the second half of 2006 .

2. Debt relief. After reaching the HIPC completion point, Zambia was granted additional debt relief under the Multilateral Debt Relief Initiative (MDRI). Effective July 1, 2006 IDA credits that had been disbursed prior to 2004 were cancelled.

\section{B. World Bank Group Strategy}

3. In March 2004, the Bank's executive Board discussed the Bank's current Country Assistance Strategy (CAS), which outlines the roadmap for the Bank's country support for the period 2004-2007. The CAS is explicitly rooted in a result-based framework, which links overall goals of the PRSP/TNDP to specific strategic objectives. World Bank activities focus on three strategic priorities:

1. Strategic Priority 1: Sustained Economic Growth Anchored in a Diversified and Export-Oriented Economy;

2. Strategic Priority 2: Improved Lives and Protection of the Vulnerable;

3. Strategic Priority 3: Efficiently and Effectively Managed Public Sector.

The Bank is in the process of preparing a new Country Assistance Strategy, which is planned to be discussed with its executive Board in the first quarter of 2008 and will be aligned with the FNDP and with the Joint Assistance Strategy for Zambia. The JASZ has been prepared by the Cooperating Partners as a response to the FNDP and the harmonization agenda as articulated in the Paris Declaration.

4. Lending operations. In addition to ongoing Bank's lending operations in the areas of public sector reform, HIV/AIDS, malaria, education, agriculture, infrastructure, support for diversification with an emphasis on tourism, and the environment, the CAS includes development policy lending. The second EMGC/DPO for US\$10 million is part of a harmonized approach to budget support and has been appraised in June 2007. The DPO is scheduled to be discussed with the Bank's executive Board in December 2007.

5. Country Diagnostic. The Bank continues to undertake substantive country diagnostics providing a solid base for policy dialogue, design, and implementation of its 
lending operations in Zambia. A combined Public Expenditure Review (PER), Country Procurement Assessment Review (CPAR), and Country Financial Accountability Assessment (CFAA), was completed in 2003 and forms the basis for the Public Expenditure and Financial Accountability TA work jointly undertaken with cooperating partners. The main economic sector works completed are a Country Economic Memorandum (CEM), which was followed by a pro-poor growth study, an Investment Climate Assessment (ICA), an Administrative Barriers study (AB), a collaborative Poverty and Vulnerability Assessment (PVA), an integrated framework study for trade (DTIS), and a Strategic Country Gender Assessment (SCGA). In addition, a Public Expenditure Review for the education has been undertaken (FY07) and a PER for the health sector is currently being finalized while an assessment of competition and regulation in Zambia's infrastructure sectors has been initiated. In FY08 a PER for the agricultural sector will be initiated as well as a Public Expenditure Tracking Survey for secondary education. An update of the Investment Climate Assessment is also under way. It is expected that the upcoming CAS will outline an analytical program for the next three to four years.

6. Projects under Supervision. IDA's active portfolio as of April 2007 contains 12 credits and grants for a total initial commitment of US\$319.5 million. During FY05, two projects were approved i.e. SEED (US\$32.2 mil.) to support economic diversification in July 2004 and the Economic Management and Growth Credit (DPL, US\$40 mil.) in Dec 2004. In FY06 three projects, the Malaria Booster Project (US\$20 mil.), Public Sector Management Project (US\$30 mil.) and Agricultural Development Support Program (US\$37.2 mil.) were approved by IDA's Board. In FY07 the Board approved a water and sanitation project (US\$23 mil.) and additional financing for the Road rehabilitation and maintenance project in the amount of US\$25 million.

7. The International Finance Corporation (IFC) is very much aligned with the Bank's policy agenda in Zambia. The IFC currently has a small portfolio of 12 projects totaling US\$15 million outstanding. These are a mix of financial sector, agribusiness, telecommunications (mobile telephone), and tourism projects. The IFC's most prominent project was for US\$30 million in the equity of Konkola Copper Mines (KCM) with AngloAmerican in 2000. The IFC exited in 2002 along with Anglo, in accordance with the terms of the investment agreement.

8. MIGA's outstanding portfolio in Zambia consists of four contracts of guarantee in the agribusiness and manufacturing services sector with a US\$36.1 million gross exposure and a US\$35.7 million net exposure as of December 2003. The projects are for the privatization, modernization, and expansion of a foundry, and for cobalt and copper facilities. They benefit the Zambian economy by saving production costs and by providing training to personnel working on projects. The estimated total amount of foreign direct investment facilitated to date is US\$155.5 million 
Table 1: Projects under Supervision

(As of August 2007)

\begin{tabular}{|c|c|c|c|c|c|c|}
\hline 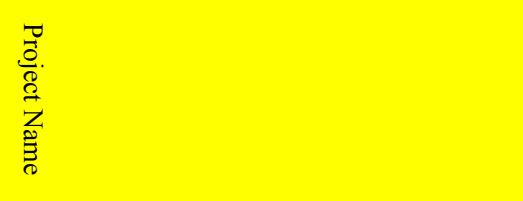 & & 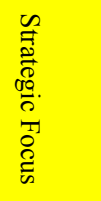 & 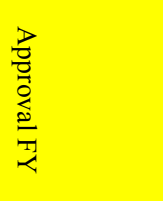 & 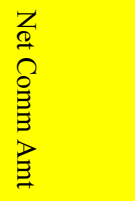 & 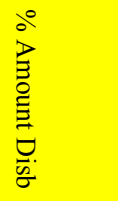 & 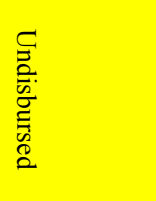 \\
\hline SEED SIL (FY05) & Credit & Pillar 1 & $07 / 29 / 2004$ & 28.2 & $37.0 \%$ & 18.4 \\
\hline GEF SEED Biodiversity & Grant & Pillar 2 & $07 / 29 / 2004$ & 4.0 & $52.3 \%$ & 1.9 \\
\hline 3A Southern Africa Power Market & Credit & Pillar 1 & $07 / 09 / 2004$ & 1.1 & $73.0 \%$ & 0.4 \\
\hline Road Rehab Maintenance Project. & Credit & Pillar 1 & $03 / 09 / 2004$ & 75.0 & $35.7 \%$ & 44.9 \\
\hline Copperbelt Environment & Credit & Pillar 1 & $03 / 20 / 2003$ & 45.0 & $37.7 \%$ & 28.3 \\
\hline HIV/AIDS-ZANARA & Grant & Pillar 2 & $12 / 30 / 2002$ & 42.0 & $89.1 \%$ & 5.5 \\
\hline TEVET & Credit & Pillar 2 & $06 / 14 / 2001$ & 25.0 & $70.1 \%$ & 8.5 \\
\hline Regional Trade Fac. Proj. - Zambia & Credit & Pillar 1 & $04 / 03 / 2001$ & 15.0 & $45.6 \%$ & 9.1 \\
\hline Malaria Booster program & Credit & Pillar 2 & $11 / 15 / 2005$ & 20.0 & $52.3 \%$ & 9.8 \\
\hline $\begin{array}{l}\text { Public Sector Management Program Support } \\
\text { Proj. }\end{array}$ & Credit & Pillar 3 & $01 / 05 / 2006$ & 30.0 & $37.3 \%$ & 19.9 \\
\hline Agricultural Development Support Program & Grant & Pillar 1 & $05 / 16 / 2006$ & 37.2 & $14.3 \%$ & 33.2 \\
\hline Water Sector Performance Improvement Proj. & Credit & Pillar 2 & $10 / 05 / 2006$ & 23.0 & 0.0 & 23.9 \\
\hline
\end{tabular}

\section{IMF-World Bank Collaboration in Specific Areas}

9. A summary of IMF-World Bank collaboration in Zambia is provided in Table 2. There are a number of areas where the Fund leads and its analysis serve as inputs into the World Bank policy formulation and advice, including policies to maintain macroeconomic stability, fiscal, monetary, and exchange rate policies. There are other areas in which the Fund and the Bank share responsibility and are coordinating closely their policy advice to the Zambian authorities, such as trade, financial sector, public expenditure management, including debt management, and economic governance. The Bank has taken the lead in the social sectors, including health, education, social protection, water and sanitation, agriculture and rural development, private sector development including regulatory issues and the environment.

10. The Fund and the Bank share joint responsibility in supporting the Government in the preparation of the PRSP and its annual progress reports on the implementation of the PRSP. The Fund and the Bank have also jointly provided assistance to Zambia for evaluating progress to reach the Completion Point under the Enhanced HIPC Initiative. In addition, the Bank and the Fund are jointly assisting GRZ to improve its debt and cash management practices. 


\section{C.1. Areas in which the World Bank leads and there is no direct IMF involvement}

11. Areas in which the Bank leads and there is no direct involvement of the IMF are support for social sectors, agriculture and rural development, economic diversification, infrastructure, and the environment with the aim of informing Zambia's next povertyreduction strategy. For the same purpose, the Bank has undertaken a Poverty and Vulnerability Assessment

12. HIV/AIDS, Malaria, and health. At this time, IDA is assisting the Ministry of Health with a US\$42 million project under a multi-sectoral AIDS program (ZANARA) and US\$20 million under its Malaria Booster project. In addition, IDA has carried out an ESW on human resources in the health sector (FY04) and is to complete a health sector PER in FY07. Building on this, IDA will provide support, but not necessarily financially for the Health Sector Support Program (SWAP) beginning in FY07. The improvement of water supplies under the Mine Township Services Project will also contribute to health outcomes in the areas served.

13. In education, the Bank is currently supporting the Ministry of Education's efforts in the area of technical education, vocational, and entrepreneurship training (TEVET). The Government is carrying out reforms aimed at improving the quality of training, making it more responsive to labor market demand and financially sustainable. Reforms also aim at increasing the participation of female trainees and trainees from socio-economically disadvantaged groups.

14. In agriculture and rural development, the Bank's focus is two fold: improvement of productivity of the sector and increase of overall food security. The Bank has reengaged in the agricultural sector given the priority Zambia attaches to this sector, as set out in the PRSP/TNDP and in the forthcoming FNDP. Indeed, in a stable macroeconomic environment, if supporting infrastructure and adequate agricultural policies are in place, a major effort could be launched to unleash growth and poverty reduction potential of the sector, in particular through well-targeted interventions for both smallholders and commercial agriculture.

15. Analytical and Advisory Activities (AAA) have been undertaken to support the formulation of an agricultural policy consistent with the planned and ongoing projects in related sectors, such as roads and electricity, to remove bottlenecks that prohibit and or make difficult for farmers to produce for the market. Hence, the policy dialogue with the Government on reforms and priorities of public spending for agriculture and rural development will address both short run policy issues, as well as a long run strategy to tap the large agricultural export potential of the country, focusing on investment in land development, irrigation, and infrastructure. A balanced treatment of smallholders and commercial agriculture is important for growth and poverty reduction. To support this effort 
an Agricultural Development Support Project has been approved in FY06 to assist the Government with these challenges.

16. The Bank supports economic diversification directly through its SEED project, which is assisting implementation of a diversification strategy in the tourism, agribusiness, and gemstone sectors. In the initial phase, the SEED project will focus on supporting the design and establishment of appropriate policy and legal frameworks to encourage private investments in these three sectors, as well as investments, primarily in tourism-related infrastructure. The tourism component will work toward making Livingstone the flagship tourism destination in Zambia and strengthening management of national parks, which underpin Zambia's medium-term tourism development strategy. The interventions in the gemstones and agribusiness subsectors are focused to strengthen the supply chain that links rural/small-scale producers to local and export markets.

17. Private sector development. To improve Zambia's investment climate, the Bank and the IFC have assisted the Government with analytical work, notably an Investment Climate Assessment and a FIAS-led Administrative Barriers to Investment study. These studies are geared to facilitate the adoption of a new and more business friendly Investment Act and result in regular consultations between business and Government to discuss how to strengthen Zambia's business climate. As a result of this dialogue, the Government, with support from the Bank and the IFC as well as other donors, prepared a private sector development strategy. The strategy was developed through an interactive process, which included intensive consultations with the private sector and other donors and coordination within the Government itself. Implementation of some of the reform actions identified in the strategy has already started through analytical support by the Bank and through programs funded by other donors.

18. In the infrastructural sectors, the Bank is assisting the authorities in the commercialization of ZESCO, Zambia's electricity conglomerate as well as its ability to build and maintain roads. Analytical work aims at identifying the main bottlenecks with regard to competition and effective service delivery. The policy recommendations and institutional changes would be supported through adjustment operations. In particular, the ongoing and proposed investment projects (Road Rehabilitation and Maintenance Project and the Water Sector Reform Project) would provide the financial resources needed for investments. Zambia will also benefit from the Southern African Power Pool Project.

19. Environmental concerns are addressed as part of each individual project as laid down in the Bank's safeguard policies. They are also addressed through the Bank's environmental project in the Copperbelt, which will assist Government with the cleanup of several hazardous sites in the Copperbelt and Kabwe area. The SEED project will assist the Government in making environmentally sustainable tourism in protected areas. 


\section{C.2. Areas in which the World Bank leads and its analysis serves as input into the IMF program}

20. The Bank leads the dialogue on institutional dimensions of public expenditure management and financial accountability as well as public sector reform and restructuring and privatization. The Bank program will support these areas through a combination of lending and analytical work. In the area of institutional dimensions of public expenditure management and financial accountability, the Bank's long-term objective is to encourage a more transparent and accountable public sector by improving budget management, procurement systems, and the accountability for public funds. The public sector reform and restructuring agenda centers on instilling a more productive and efficient public service. To achieve this goal, reform efforts aim at promoting a more qualified and motivated civil service, developing a strategic management orientation in line ministries, improving policy coordination and implementation, and lastly, strengthening local government's capacity to participate in the development process.

21. While the Bank has taken the lead in privatization, the IMF has also a strong interest in these areas since many of the reforms are critical to achieving macroeconomic stabilization and enhancing Zambia's growth prospects. Accordingly, there is a high degree of consultation and coordination between the two institutions on these matters.

\section{C.3. Areas of shared responsibility}

22. The Fund and the Bank are working jointly in the following five areas (i) trade policy; (ii) financial sector reform; (iii) debt management and domestic debt market development; (iv) PRSP monitoring and evaluation; and preparation of a LIC debt sustainability analysis.

23. In the area of trade policy, the Bank, with collaboration from other partners, will undertake an integrated framework exercise. The Fund and the Bank jointly reviewed the Financial Sector Development Plan (FSDP). Follow ups on the FSAP and the FSDP will guide the Bank's policy dialogue and interventions in the financial sector in close collaboration with the IMF. The PRGF and the Economic Management and Growth Credit assist the Government on the resolution of three state-owned Non-Bank Financial Institutions and the strengthening of the operational independence of the Bank of Zambia. An FSAP update is planned for 2007.

\section{Assistance in the areas of central government debt management and domestic} debt market development will focus on reducing vulnerability and the cost of and the need for domestic financing. The Bank, through its Treasury department, has under taken a debt management and debt market development assessment (FY05) and currently assists the Government in putting the recommended institutional changes in place. 
25. The Fund and the Bank are working closely to review progress with Zambia's PRSP. The Bank and the Fund jointly prepared the Joint Staff Assessment Note (JSAN) on the third annual progress report in December 2006 and are in the process of finalizing the JSAN for the Zambia's second PRSP i.e. the Fifth National Development Plan. Finally, the Fund and the Bank jointly prepared a LIC DSA in late 2005. Zambia reached HIPC completion in April 2005 and its completion point document contained also debt sustainability analysis.

\section{C.4. Areas in which the Fund leads and its analysis serves as input into the World Bank program}

26. The Fund leads the dialogue on macroeconomic framework, in particular with regard to fiscal policy, advising the Government on the overall envelope for public expenditures, tax policy, as well as monetary and exchange rate policy. In these areas the Bank takes into account the policy recommendations of the Fund and ensures that its own policy advice, embedded in its development policy operation (the Economic Management and Growth Credit), is consistent. 
Table 2: IMF-World Bank Collaboration in Zambia (FY03-FY08)

\begin{tabular}{|c|c|c|c|}
\hline CAS Component & $\begin{array}{l}\text { Specialized advice } \\
\text { from Fund }\end{array}$ & $\begin{array}{l}\text { Specialized advice } \\
\text { from World Bank }\end{array}$ & Key Instruments \\
\hline \multicolumn{4}{|c|}{ Goal: A growth conducive macro economic environment } \\
\hline $\begin{array}{l}\text { Subject: Economic } \\
\text { Framework/ } \\
\text { Management }\end{array}$ & $\begin{array}{l}\text { Monetary policy; } \\
\text { Financial sector } \\
\text { reforms, exchange } \\
\text { rate, fiscal policy } \\
\text { and wage bill, debt } \\
\text { management, } \\
\text { balance of } \\
\text { payments, } \\
\text { economic statistics }\end{array}$ & $\begin{array}{l}\text { Debt management; } \\
\text { payroll management; } \\
\text { Financial sector } \\
\text { reforms }\end{array}$ & $\begin{array}{l}\text { IMF: PRGF performance criteria and } \\
\text { benchmarks on key monetary and fiscal } \\
\text { targets and structural reforms } \\
\text { Bank: Ongoing portfolio: PSMP (FY06) } \\
\text { New Lending: EMGC II (DPL) (FY08) } \\
\text { ESW: Debt Management Assessment } \\
\text { Jointly: JSAN on PRSP progress report and } \\
\text { new PRSP, and debt sustainability analysis }\end{array}$ \\
\hline \multicolumn{4}{|c|}{ Goal: A diversified and export oriented economy } \\
\hline $\begin{array}{l}\text { Subject: Private } \\
\text { sector development; } \\
\text { Trade and export } \\
\text { diversification }\end{array}$ & $\begin{array}{l}\text { Financial sector } \\
\text { reform; trade } \\
\text { policy; exchange } \\
\text { rate policy }\end{array}$ & $\begin{array}{l}\text { Economic growth, } \\
\text { investment climate } \\
\text { for private sector } \\
\text { (infrastructure } \\
\text { services, reforms of } \\
\text { financial sector and } \\
\text { pension funds, power } \\
\text { and } \\
\text { telecommunication } \\
\text { sector reforms, } \\
\text { vocational training), } \\
\text { trade policy for } \\
\text { export } \\
\text { diversification, rural } \\
\text { development, } \\
\text { environmental } \\
\text { regulations for the } \\
\text { mining sector }\end{array}$ & $\begin{array}{l}\text { IMF: PRGF performance criteria and } \\
\text { benchmarks on key monetary and fiscal } \\
\text { targets and structural reforms. } \\
\text { Bank: On-going Portfolio: Regional Trade } \\
\text { Facilitation Project (FY00), TEVET (FY00), } \\
\text { South Africa Power Pool (FY04) SEED } \\
\text { (FY05), PMSP (FY06), ASDP (FY06), Water } \\
\text { Sector Improvement Project (FY07). } \\
\text { New Lending: EMGC II (DPL-FY08), and } \\
\text { Increased Access to Electricity (FY08), } \\
\text { ESW: Country Economic Memorandum } \\
\text { (FY04), Investment Climate Assessment } \\
\text { (FY04), ICT Options Study (FY06), Elements } \\
\text { of effective regulations (FY07), trade in } \\
\text { services (FY07) } \\
\text { Jointly: FSAP (FY02) FSAP update } \\
\text { (FY07/08), JSAN of PRSP progress report } \\
\text { and new PRSP }\end{array}$ \\
\hline \multicolumn{4}{|c|}{ Goal: Better health and long-term survival, with particular focus on at risk and vulnerable groups } \\
\hline Subject: Health & & $\begin{array}{l}\text { Health Sector } \\
\text { Expenditure } \\
\text { Management. }\end{array}$ & $\begin{array}{l}\text { Bank: On-going Portfolio: ZANARA (FY03), } \\
\text { Malaria Booster Project (FY06), Water Sector } \\
\text { Improvement Project (FY07); } \\
\text { ESW: Health Sector PER (FY07), Strategic } \\
\text { Country Gender Assessment (FY04) }\end{array}$ \\
\hline \multicolumn{4}{|c|}{ Goal: Reverse the spread of HIV/AIDS: decrease prevalence among it citizens } \\
\hline Subject: HIV/AIDS & & $\begin{array}{l}\text { Behavioral change; } \\
\text { education; } \\
\text { prevention, care and } \\
\text { risk mitigation } \\
\text { services. }\end{array}$ & $\begin{array}{l}\text { Bank: } \text { On-going Portfolio: ZANARA (FY03); } \\
\text { ESW: Social Safety Nets and Protection } \\
\text { Strategy Note (FY04), Strategic Country } \\
\text { Gender Assessment (FY04) }\end{array}$ \\
\hline
\end{tabular}




\begin{tabular}{|c|c|c|c|}
\hline Subject: Education & & $\begin{array}{l}\text { Provision and quality } \\
\text { of primary education }\end{array}$ & $\begin{array}{l}\text { Bank: On-going Portfolio: TEVET(FY01) } \\
\text { ESW: Education Sector PER(FY05) }\end{array}$ \\
\hline \multicolumn{4}{|c|}{ Goal: Improved living conditions for poor/vulnerable households in drought prone areas } \\
\hline $\begin{array}{l}\text { Subject: Social } \\
\text { Protection/ Risk } \\
\text { Mitigation }\end{array}$ & & $\begin{array}{l}\text { Social protection and } \\
\text { risk management } \\
\text { systems }\end{array}$ & $\begin{array}{l}\text { IMF: PRGF } \\
\text { Bank: Ongoing Portfolio: SEED (FY04) } \\
\text { Water Sector Improvement Project (FY07): } \\
\text { ESW: Poverty and Vulnerability Assessment, } \\
\text { Strategic Country Gender Assessment, Social } \\
\text { Safety Nets and Social Protection Strategy } \\
\text { Note, Health Sector Review }\end{array}$ \\
\hline \multicolumn{4}{|c|}{ Goal: Good governance and public sector management } \\
\hline $\begin{array}{l}\text { Subject: Public } \\
\text { Expenditure }\end{array}$ & $\begin{array}{l}\text { Medium-term } \\
\text { budget framework, } \\
\text { tax policy and } \\
\text { administration. } \\
\text { Arrears and } \\
\text { commitment } \\
\text { control }\end{array}$ & $\begin{array}{l}\text { Public expenditure } \\
\text { analysis, } \\
\text { Capacity building in } \\
\text { financial } \\
\text { management and } \\
\text { accountability }\end{array}$ & $\begin{array}{l}\text { IMF: PRGF measures to put in place the } \\
\text { IFMIS and to ensure timely reporting on } \\
\text { budget execution } \\
\text { Bank: On-going Portfolio: PSMP (FY06), } \\
\text { New Lending: EMGC II (FY08), Local } \\
\text { Development Project (FY08); } \\
\text { ESW: PEMFAR (FY03); } \\
\text { Jointly: HIPC AAP expenditure tracking } \\
\text { assessment (FY04) }\end{array}$ \\
\hline \multicolumn{4}{|c|}{ Goal: A productive and efficient public service } \\
\hline $\begin{array}{l}\text { Subject: Public } \\
\text { Sector Reform and } \\
\text { Restructuring }\end{array}$ & & $\begin{array}{l}\text { Pay reform, public } \\
\text { sector restructuring }\end{array}$ & $\begin{array}{l}\text { Bank: } \text { On-going Portfolio: PSMP (FY06) } \\
\text { New Lending: EMGC II (FY08), Local } \\
\text { Development Project (FY08) } \\
\text { ESW: PEMFAR(FY03), Governance Survey }\end{array}$ \\
\hline
\end{tabular}

Questions may be referred to Jos Verbeek (202-473-5539). 


\section{ANNEX IV. ZAMbia: Statistical ISSUES}

1. Data are generally adequate for surveillance purposes, but there are serious shortcomings in the national accounts, balance of payments, and consumer prices. The present arrangements for compiling macroeconomic statistics involve duplication of effort and insufficient coordination among the Bank of Zambia (BoZ), the Ministry of Finance and National Planning (MFNP), and the Central Statistical Office (CSO). The situation largely reflects resource constraints and organizational weakness within the CSO that have affected its ability to produce economic statistics on a timely basis.

2. Resource constraints have also hampered the capacity to absorb technical assistance (TA) in statistics. A STA mission visited Lusaka in October 2006 to discuss ways to enhance the effectiveness of technical assistance. Discussions with the authorities and donors focused on the need to implement a number of the 2004 data ROSC mission's recommendations and address the inadequate resources that have hampered the effectiveness of TA, particularly in the area of balance of payments statistics. The authorities committed to augment resources to support the compilation and dissemination of national accounts, prices, balance of payments and government finance statistics (GFS) and implementation of outstanding data ROSC mission recommendations.

3. As one of 22 countries participating in the Fund's General Data Dissemination System (GDDS) Project for Anglophone African Countries, Zambia has undertaken to use the GDDS as a framework for the development of its national statistical system. Zambia is participating in the national accounts and the GDDS/PRSP modules of the Anglophone Africa project (funded by the U.K. Department for International Development (DFID)). This project aims to assist participating countries to implement plans for improvement identified in the metadata and to meet GDDS recommended statistical practices. Zambia's metadata were posted on the Dissemination Standards Bulletin Board on November 1, 2002 and partially updated in June 2006.

\section{Real sector statistics}

4. The national accounts estimates are compiled according to the conceptual guidelines of the $1968 S N A$, but a phased approach to the introduction of the $1993 S N A$ is planned. For the production approach, 1994 is the latest benchmark year for value-added ratios. For many important industry areas, such as wholesale and retail trade, construction, business services and many other service industries, consisting mainly of small-scale private service providers, there are no appropriate indicators. Data on total production and intermediate consumption of establishments are not available. On the expenditure side, there are no reliable indicators of household consumption and private final consumption expenditure is derived residually. Source data for estimating gross fixed capital formation and changes in stocks are incomplete. 
5. An IMF/DFID GDDS mission that visited Lusaka during February 2006 noted that the CSO had obtained data comprehensive enough to enable it to produce a new benchmark for 2003. A follow-up mission in July 2006, however, found little progress producing one. The authorities plan to conduct a comprehensive economic census to complete the rebasing of the national accounts in 2007.

6. Current price estimates for both production and expenditure have been derived from the constant price indicators using wholesale price indices (WPIs) and consumer price indices (CPIs) as deflators. However, for the most recent period, the WPI were not available and the CPI components were used throughout the system.

7. The CPI broadly adheres to international methodological standards. The classification system used for compilation closely follows the Classification of Individual Consumption by Purpose. However, the frequency of the household budget surveys has been insufficient to capture changes in consumer expenditure structure on a timely basis. The authorities plan to launch a new rebased CPI in 2008.

\section{Government finance statistics}

8. In 2007, the authorities made progress with improving reporting Government Finance Statistics for publication in the GFSY. They submitted a consistent time series of GFS data, covering only the budgetary central government, for 2001 through 2007, based on the GFSM 2001 framework. The transactional coverage of these data is limited to government operations (revenue, expense) and transactions in nonfinancial assets. Data on transactions in financial assets and liabilities are still unavailable. No monthly or quarterly fiscal data are reported for publication in International Financial Statistics (IFS). The authorities report monthly budget data to AFR for operational use in a timely manner, but the data are often subject to substantial revisions, and data on extra-budgetary institutions and local governments are not available.

9. An IMF/DFID GDDS mission visited Lusaka in March 2003 to provide technical assistance to the MFNP on government finance statistics and to advise on migration to the methodology of the Government Finance Statistics Manual 2001 (GFSM2001).

10. An IMF/GDDS mission visited Lusaka again in May 2005 to assess the overall quality, coverage, and timeliness of fiscal data. The mission found that decentralization of government activities in earlier years had led to the creation of numerous extra-budgetary institutions. While the majority of these entities follow international accounting practices, they are not obliged to report to the MFNP. Currently, an estimated 35 percent of government activities are not captured in the data. The mission also noted that the implementation of Integrated Financial Management and Information System (IFMIS) is a long-term project with an expected completion date of 2009. 


\section{Monetary and financial statistics}

11. In 2003, the BoZ migrated to a new computing system for generating its monthly trial balance, which makes it possible to produce monetary accounts on a timely basis. The BoZ has also eliminated discrepancies between its records and those of the IMF's Finance Department and it now publishes a monetary survey on a monthly basis. Monetary data are reported to STA using standardized report forms; these data have been published in the IFS Supplement since September 2006.

12. An April- May 2005 monetary statistics mission found that the authorities had implemented most of the previous recommendations by STA with one notable exception, overdrafts were still being netted against deposits. The institutional coverage of other depository corporations has been expanded to include commercial banks (including three banks in liquidation), building societies, and the National Savings and Credit Bank. Data reported by commercial banks are considered generally adequate, but there are some problems in the sectorization of public sector deposits, resulting in some central government deposits in national and foreign currencies being included in the definition of broad money. Also, some other depository corporations record repurchase agreements as the outright buying or selling of the securities, rather than as collateralized loans. Deposits of three banks that are currently in the process of liquidation are classified as restricted deposits and excluded from broad money.

\section{External sector statistics}

13. Balance of payments statistics are compiled and disseminated by the $\mathrm{BoZ}$ in accordance with the fifth edition of the Balance of Payments Manual. However, the CSO is legally responsible for compiling all macroeconomic statistics. Reporting of balance of payments statistics for publication by the IMF Statistics Department remains problematic. The most recent balance of payments data reported to STA are for 2000. International investment position (IIP) data are not compiled.

14. The 2004 data ROSC mission found data sources poor and unreliable, while compilers were using inadequate methodologies. Balance of payments data contain serious omissions, including some affecting the important mining sector. Furthermore, it found that resources (including staffing) were inadequate for the task of compiling good quality balance of payments data. The ability to absorb further technical assistance was also found compromised due to the aforementioned weakness. In October 2006, the authorities agreed to provide more staff for the compilation of balance of payments statistics to ensure better absorption capacity prior to the provision of additional technical assistance in this area.

15. Significant gaps in data exist in various areas including reinvested earnings, trade credits, and export proceeds held abroad by mining and nontraditional exporters. While the source data are generally adequate in terms of timeliness, they fall short in terms of 
coverage. As a result the shortcomings in source data coverage, indirect estimation methods are used, some of which have remained unchanged for many years and are out of date. Also, data on services, income and transfers are incomplete and valuation methods need to be improved. Source data on private sector foreign assets and liabilities are insufficient to compile an IIP statement. 
As of August 21, 2007

\begin{tabular}{|c|c|c|c|c|c|c|c|}
\hline & \multirow{2}{*}{$\begin{array}{c}\text { Date of } \\
\text { latest } \\
\text { observation }\end{array}$} & \multirow[t]{2}{*}{ Date received } & \multirow{2}{*}{$\begin{array}{c}\text { Frequenc } \\
\text { y of } \\
\text { Data }^{6}\end{array}$} & \multirow{2}{*}{$\begin{array}{c}\text { Frequenc } \\
\text { y of } \\
\text { Reporting } \\
6\end{array}$} & \multirow{2}{*}{$\begin{array}{l}\text { Frequency } \\
\text { of } \\
\text { publication } \\
6\end{array}$} & \multicolumn{2}{|c|}{ Memo Items: } \\
\hline & & & & & & $\begin{array}{c}\text { Data Quality - } \\
\text { Methodological } \\
\text { soundness }{ }^{7}\end{array}$ & $\begin{array}{l}\text { Data Quality - Accuracy } \\
\quad \text { and reliability }\end{array}$ \\
\hline Exchange Rates & Sep. 2007 & Oct. 12, 2007 & $\mathrm{D}$ & $\mathrm{D}$ & $\mathrm{D}$ & & \\
\hline $\begin{array}{l}\text { International Reserve Assets and Reserve Liabilities } \\
\text { of the Monetary Authorities } 1\end{array}$ & Sep. 2007 & Oct. 15, 2007 & W & W & M & & \\
\hline Reserve/Base Money & Aug. 2007 & Oct. 19, 2007 & W & W & $\mathrm{F}$ & \multirow{4}{*}{ LO, LO, LO, LO } & \multirow{4}{*}{ LO, O, O, O, O } \\
\hline Broad Money & Aug. 2007 & Oct. 19, 2007 & M & M & M & & \\
\hline Central Bank Balance Sheet & Aug. 2007 & Oct. 19, 2007 & M & M & M & & \\
\hline Consolidated Balance Sheet of the Banking System & Aug. 2007 & Oct. 19, 2007 & M & M & M & & \\
\hline Interest Rates ${ }^{2}$ & Aug. 2007 & Oct. 3, 2007 & W & W & $\mathrm{F}$ & & \\
\hline Consumer Price Index & Jul. 2007 & Oct. 19, 2007 & M & M & M & O, LO, O, LO & LNO, LO, LO, LNO, LO \\
\hline $\begin{array}{l}\text { Revenue, Expenditure, Balance and Composition of } \\
\text { Financing }{ }^{3}-\text { General Government }\end{array}$ & NA & NA & & & & \multirow[t]{2}{*}{ LO, LNO, LNO, LO } & \multirow[t]{2}{*}{ LNO, LO, O, O, LNO } \\
\hline $\begin{array}{l}\text { Revenue, Expenditure, Balance and Composition of } \\
\text { Financing }{ }^{3}-\text { Central Government }\end{array}$ & Dec. 2006 & Mar. 2007 & M & M & M & & \\
\hline $\begin{array}{l}\text { Stocks of Central Government and Central } \\
\text { Government-Guaranteed Debt }\end{array}$ & Dec. 2005 & Apr. 21, 2006 & M & M & A & & \\
\hline External Current Account Balance & 2006 & Mar. 2007 & A & A & A & \multirow[t]{2}{*}{ LO, LNO, LNO, O } & \multirow[t]{2}{*}{ LNO, O, LNO, LO, LNO } \\
\hline Exports and Imports of Goods and Services & Dec. 2006 & Mar. 2007 & Q & Q & $A$ & & \\
\hline GDP/GNP & 2006 & Mar. 2007 & A & A & A & LO, LO, LO, LO & LNO, LO, LNO, LNO, LNO \\
\hline Gross External Debt & Sep. 2005 & Mar. 2006 & $Q$ & I & I & & \\
\hline
\end{tabular}

Includes reserve assets pledged or otherwise encumbered as well as net derivative positions.

${ }^{2}$ Both market-based and officially-determined, including discounts rates, money market rates, rates on treasury bills, notes and bonds.

${ }^{3}$ Foreign, domestic bank, and domestic nonbank financing.

${ }^{4}$ The general government consists of the central government (budgetary funds, extra budgetary funds, and social security funds) and state and local governments.

${ }^{5}$ Including currency and maturity composition.

${ }^{6}$ Daily (D), Weekly (W), Monthly (M), Quarterly (Q), Annually (A); Irregular (I); or Not Available (NA).

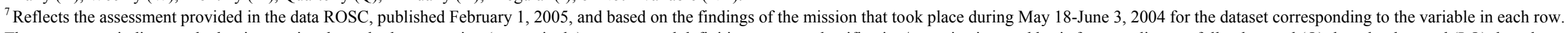

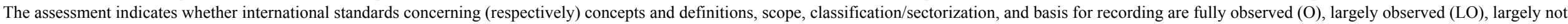
observed (LNO), or not observed (NO).

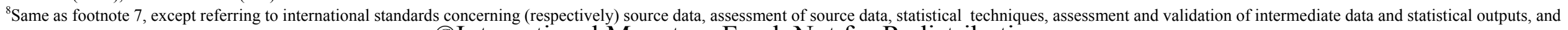
revision studies. 


\section{Statement by the IMF Staff Representative \\ December 7, 2007}

1. This statement provides information that has become available since the staff report was issued to the Board. The thrust of the staff appraisal remains unchanged.

2. Macroeconomic indicators remain robust. Inflation fell further in November, to 8.7 percent year-on-year, mostly on account of lower growth in food prices. International reserves have continued to increase and by mid-November 2007 exceeded US\$900 million (2.5 months of imports). While the kwacha has remained stable against the US dollar since mid-summer, the real and nominal effective rate depreciated by around 3 percent from July to September 2007. Annual broad money growth slowed to 24 percent at end-September 2007 from 36 percent at end-June 2007.

3. Reserve money growth has remained volatile. While reserve money declined as projected immediately following the reduction in reserve requirements on October 1, in recent weeks, reserve money has risen to well above the end-year projection.

4. Revenue performance remained strong through October. Revenue collections were 0.2 percent of GDP higher than projected, because of higher income tax collections. While current expenditures were broadly in line with the budget, releases for domesticallyfinanced capital expenditure continued to record a shortfall relative to the revised budget projection. Based on preliminary information, the overall fiscal position through October continued to be in surplus due to slow implementation of domestically-financed capital projects.

\section{A recently published living conditions survey indicates a decline in overall} poverty. The poverty headcount declined from 68 percent of the population in 2004 to 64 percent in 2006 . While poverty in the urban areas declined significantly, the survey shows a slight increase in poverty rates in the rural areas. 


\section{INTERNATIONAL MONETARY FUND}

\section{IMF Executive Board Concludes 2007 Article IV Consultation with Zambia}

On December, 7, 2007, the Executive Board of the International Monetary Fund (IMF) concluded the Article IV consultation with Zambia. ${ }^{1}$

\section{Background}

The Zambian economy has performed well in recent years because of stronger macroeconomic policies, a favorable external environment, and extensive debt relief. The economic expansion has been broad-based and has benefited greatly from the revival of the mining sector and high copper prices. Public finances have improved markedly and inflation has been brought under control. The external position has strengthened significantly, mainly due to booming copper export receipts and debt relief through the HIPC Initiative and MDRI, which have contributed to a substantial real appreciation of the exchange rate and a buildup in international reserves. Poverty has been reduced, but remains high; and while progress has been made in some areas, the achievement of the Millennium Development Goals remains a challenge.

The positive economic performance of recent years continued in 2006 and the first half of 2007. Real GDP accelerated to 6.2 percent in 2006 , driven by a vigorous expansion in mining, construction and telecommunications. Inflation, after falling to single digits in 2006, picked up in

\footnotetext{
${ }^{1}$ Under Article IV of the IMF's Articles of Agreement, the IMF holds bilateral discussions with members, usually every year. A staff team visits the country, collects economic and financial information, and discusses with officials the country's economic developments and policies. On return to headquarters, the staff prepares a report, which forms the basis for discussion by the Executive Board. At the conclusion of the discussion, the Managing Director, as Chairman of the Board, summarizes the views of Executive Directors, and this summary is transmitted to the country's authorities.
} 
early 2007, but tighter monetary conditions and a turnaround in the kwacha brought it back to single digits (9 percent) in October.

Fiscal consolidation has continued. Revenue outperformed projections through September 2007, reflecting higher income tax payments from mining companies. Expenditures, however, were below projections owing to a large shortfall in releases for capital projects and because the released funds were not fully spent due to delays in tender procedures. The overall balance (including grants) was 0.8 percent of GDP through September-about $2 \frac{1}{2}$ percentage points of GDP higher than projected.

The rate of monetary expansion has fluctuated widely, in part due to difficulties in managing the monetary and exchange rate consequences of variations in capital inflows. On October 1 , the statutory reserve requirement was reduced from 14 percent to 8 percent, injecting additional liquidity into the banking system and necessitating offsetting measures to contain monetary growth. Nominal interest rates on government securities have trended upward since late 2006, while real rates have risen moderately since inflation peaked earlier in the year.

The external position has strengthened considerably. The current account deficit (excluding grants) declined markedly to 1.3 percent of GDP in 2006, as copper export receipts doubled and nontraditional exports increased by 30 percent. Export receipts remained strong in the first half of 2007. The strong external position and the tight fiscal conditions contributed to a build up of international reserves to about 2.4 months of imports at end-June 2007.

\section{Executive Board Assessment}

Directors commended the Zambian authorities for pursuing sound macroeconomic policies, which, together with high copper export prices and debt relief, have contributed to a strong economic performance. Economic growth is robust, inflation has been brought under control, and public finances and the external position have both improved markedly.

Directors encouraged the authorities to take advantage of the favorable outlook to step up their efforts to further strengthen growth and reduce poverty in the context of the Fifth National Development Plan. The focus should be on improving budget execution and creating fiscal space for increased spending on infrastructure and social sectors; strengthening project evaluation and implementation capacity; diversifying the economy to reduce the vulnerability to fluctuations in copper prices; and further improving the conditions for private sector growth. Managing the effects of high foreign exchange inflows will be key to maintaining macroeconomic stability.

Directors commended the reduction of inflation to single-digit levels, and recommended a continued tight monetary policy in the period ahead. Directors noted the complications for liquidity management that could arise from weaknesses in budget execution and the recent lowering of statutory reserve requirements. In this respect, they welcomed the authorities' decision to postpone some nonpriority project spending in the last quarter of the year, and the 
transfer of government deposits from commercial banks to the central bank. Foreign exchange sales to mop up excess liquidity could also be considered, although Directors recognized that this should be balanced against the need for a further modest build-up of international reserves to enhance the country's ability to weather external shocks. Going forward, strengthened coordination between fiscal and monetary policy, and the further development of the financial system, would be important steps to improve liquidity management.

Directors believed that the authorities' managed float exchange rate policy continues to serve Zambia well. They welcomed the assessment that the current exchange rate is consistent with external stability and aligned with economic fundamentals, including a significant improvement in the terms of trade.

Directors commended the authorities' disciplined fiscal stance, and welcomed the targeted increase in revenues and shift towards capital spending and arrears reduction in the preliminary 2008 budget. They noted that the projected growth in the government wage bill heightens the need to accelerate civil service and pay reform. Directors emphasized the importance of strengthening tax administration and widening the tax base, and welcomed the efforts underway to increase revenue by aligning the fiscal regime for mining companies with international standards.

Directors saw further progress on strengthening public expenditure management as crucial to enhancing the effective use of public resources. They encouraged the authorities to step up implementation of the public expenditure management and financial accountability reform program, including establishing a treasury single account and implementing the integrated financial management and information system. Directors stressed the importance of aligning the budget cycle with the fiscal year and finalizing the new procurement act in order to improve budget execution.

Directors were encouraged that the risk of external debt distress in Zambia is currently low. They commended the authorities' efforts to strengthen debt management, and their cautious approach to external borrowing, which focuses on concessional loans. While acknowledging that, in the absence of concessional resources, some nonconcessional external borrowing could be considered for projects that are economically viable, Directors cautioned that such borrowing should not jeopardize debt sustainability. In particular, it would be important to complement the debt strategy with measures to improve capacity to evaluate investment projects.

Directors welcomed the progress made on financial sector reform, and supported further efforts to deepen the financial sector and lower intermediation costs. They encouraged the authorities to develop an active secondary market in government securities, further improve the legal and regulatory framework for financial activity, and broaden the availability of financial services.

Directors called for accelerated implementation of reforms to improve the business environment and to enhance productivity and competitiveness. They welcomed the authorities' commitment to an open trade regime to accelerate export development. Directors considered the development of the energy sector to be vital to achieving a vibrant private sector and reducing 
poverty. They emphasized the importance of raising electricity tariffs to levels consistent with full cost recovery, and of strengthening the corporate governance and efficiency of the public utility. Directors welcomed the progress made toward participation in the Extractive Industries Transparency Initiative.

Public Information Notices (PINs) form part of the IMF's efforts to promote transparency of the IMF's views and analysis of economic developments and policies. With the consent of the country (or countries) concerned, PINs are issued after Executive Board discussions of Article IV consultations with member countries, of its surveillance of developments at the regional level, of post-program monitoring, and of ex post assessments of member countries with longer-term program engagements. PINs are also issued after Executive Board discussions of general policy matters, unless otherwise decided by the Executive Board in a particular case. 
Zambia: Selected Economic Indicators (2004-07)

\begin{tabular}{|c|c|c|c|c|}
\hline & 2004 & 2005 & 2006 & $\begin{array}{l}2007 \\
\text { Proj. }\end{array}$ \\
\hline & \multicolumn{4}{|c|}{ (In percent changes; unless otherwise indicated) } \\
\hline \multicolumn{5}{|l|}{ National account and prices } \\
\hline GDP at constant prices & 5.4 & 5.2 & 6.2 & 6.2 \\
\hline GDP deflator & 20.5 & 18.6 & 13.8 & 10.0 \\
\hline $\begin{array}{l}\text { GDP at market prices } \\
\text { (In billions of kwacha) }\end{array}$ & 25,997 & 32,456 & 39,223 & 45,849 \\
\hline \multicolumn{5}{|l|}{ Consumer prices (average) } \\
\hline Headline & 18.0 & 18.3 & 9.0 & 10.8 \\
\hline Underlying (excluding food) & 19.8 & 18.1 & 13.6 & 16.5 \\
\hline Consumer prices (end of period) & 17.5 & 15.9 & 8.2 & 9.0 \\
\hline \multicolumn{5}{|l|}{ External sector } \\
\hline Terms of trade (deterioration -) & 34.6 & 6.8 & 54.8 & 5.4 \\
\hline Average exchange rate (kwacha per U.S. dollar) & 4,779 & 4,464 & 3,601 & $\ldots$ \\
\hline (in percentage change; depreciation -) & -1.0 & 6.6 & 19.3 & $\ldots$ \\
\hline Real effective exchange rate (depreciation -$)^{1}$ & 3.0 & 24.1 & 32.3 & $\ldots$ \\
\hline \multicolumn{5}{|l|}{ Money and credit (end of period) } \\
\hline Domestic credit to the private sector & 47.7 & 18.7 & 54.3 & 35.0 \\
\hline Reserve money $^{2}$ & 21.1 & 10.2 & 29.7 & -12.6 \\
\hline \multirow[t]{2}{*}{ M3 } & 30.3 & 0.4 & 45.1 & 17.7 \\
\hline & \multicolumn{4}{|c|}{ (In percent of GDP) } \\
\hline \multicolumn{5}{|l|}{ National accounts } \\
\hline Gross investments & 23.0 & 22.5 & 22.6 & 24.1 \\
\hline Government & 8.7 & 7.0 & 4.1 & 5.7 \\
\hline Private & 14.3 & 15.5 & 18.5 & 18.4 \\
\hline National savings & 16.0 & 17.4 & 25.5 & 20.1 \\
\hline Gross foreign savings & 7.0 & 5.1 & -2.9 & 4.0 \\
\hline \multicolumn{5}{|l|}{ Central government budget ${ }^{3}$} \\
\hline Overall balance & -0.8 & -2.6 & 18.6 & -0.6 \\
\hline (excluding grants) & -6.3 & -8.3 & -7.4 & -5.4 \\
\hline Revenue & 18.2 & 17.4 & 16.9 & 18.4 \\
\hline Grants & 5.5 & 5.6 & 26.0 & 4.9 \\
\hline Total expenditure & 24.5 & 25.7 & 24.3 & 23.8 \\
\hline \multicolumn{5}{|l|}{ External sector } \\
\hline \multicolumn{5}{|l|}{ Current account balance } \\
\hline (including official grants) & -7.0 & -5.1 & 2.9 & -4.0 \\
\hline \multirow[t]{2}{*}{ (excluding official grants) } & -12.2 & -10.9 & -1.3 & -8.8 \\
\hline & \multicolumn{4}{|c|}{ (In percent of export of goods and services) } \\
\hline NPV of external public debt (including IMF) & 176 & 80 & 16 & 14 \\
\hline
\end{tabular}

Sources: Zambian authorities; and IMF staff estimates and projections.

${ }^{1}$ Excludes Zimbabwe.

${ }^{2}$ The projected reduction in reserve money for December 2007 reflects the lowering of statutory reserve requirements from 14 to 8 percent on October 1, 2007.

${ }^{3}$ Grants in 2006 include MDRI debt cancellation amounting to 21.4 percent of GDP. 


\section{Statement by Peter Gakunu, Executive Director for Zambia and Dieudonne Nintunze, Senior Advisor to Executive Director. December 7, 2007}

1. The Zambian authorities are appreciative of the continued support by the Executive Board and Management. They value the constructive policy dialogue and advice given by staff under the program, and the candid exchange of views during the last discussions in Lusaka under the 2007 Article IV Consultation, and welcome the comments and recommendations in the well balanced report and analytical insights contained in the selected issues paper.

\section{Recent economic developments and prospects}

2. Over the recent years, the Zambian authorities have commendably pursued strong economic and financial policies that have contributed to the improved economic conditions and sustaining macroeconomic stability. Deepened implementation of the structural reform agenda, notably the public sector reform, financial sector development plan, and private sector development initiative, has resulted in robust and broad-based growth, improved external position, and a recovery in employment creation. In addition, the poverty level, though still high, has started to trend downward. For the fifth consecutive year, the Zambian economy registered impressive performance, and the outlook is favorable. Real GDP growth picked up to 6.2 percent in 2006 led by strong activity in mining, manufacturing, construction, services, and other sectors, and is expected to continue expanding by 6-7 percent on average per year over the medium-term. Inflation was contained at its lowest levels in three decades, and the authorities' objective is to contain it further to lower single digits in the medium-term. The level of international reserves has also strengthened over the recent years, as external sector conditions improved substantially supported by increases in copper export receipts and expansion of non-traditional exports, as well as extensive debt relief.

3. The authorities remain committed to fiscal consolidation, with revenue collection expected to reach 18.4 percent of GDP in 2007 and should continue improving in the medium-term, fueled by reforms in the tax system, improved tax administration, and higher income tax payments from mining companies. Primary expenditures were contained in 2006 below the program target, while important social programs were protected from spending cuts, in particular the objective of recruiting additional teachers and health workers. Spending on domestically financed poverty-reducing programs was absorbed at 6.0 percent of GDP and is expected to further increase to 8.3 percent of GDP in 2007. Nonetheless, the authorities are aware of the existing difficulties in budget execution, including under 
expenditure on capital projects and associated delays in tenders. As a result, expenditures were below projection at end-September 2007, and government deposits at the Bank of Zambia (BoZ) increased accordingly. The authorities are committed to reinforce implementation of the Public Expenditure Management and Financial Accountability Reform (PEMFA) which will contribute to address the budget execution rigidities.

4. The BoZ intensified its efforts of containing excess liquidity in the banking system through its open market operations and auctions of government securities, with a view to attaining its objective of low levels of inflation. In this connection, the reserve money growth, which had accelerated in 2006, slowed down in June 2007. The authorities are aware of liquidity pressures stemming from speeding up of budget execution in the last months of 2007, and the recent reduction in cash reserve requirement in October 2007. To this end, they are initiating appropriate measures, including postponement of part of the budget releases for non-priority projects and transfer of government deposits from commercial banks to the BOZ.

5. The exchange rate is aligned with macroeconomic fundamentals, and domestic and financial policies are consistent with external stability. Financial conditions and performance of the banking sector are broadly satisfactory. Capital and reserves are in compliance with the prudential requirements, and the banking sector is well capitalized, while the asset quality, earnings and liquidity ratios remain at sound levels.

\section{Medium-term policy challenges and strategies}

6. Notwithstanding the impressive economic performance and positive medium-term outlook, the authorities are concerned that Zambia may not be able to achieve the MDGs. The main challenges include the need to further consolidate macroeconomic stability and diversify the economy, with a view to sustaining high economic growth and enhancing employment and income opportunities for the population. The authorities are committed to strengthening implementation of their Fifth National Development Plan (FNDP) to achieve their objectives of creating fiscal space, strengthening absorptive capacity of development spending, managing macroeconomic effects of increased foreign exchange inflows, and further improving conditions for private sector growth.

\section{Fiscal policy}

7. In the FNDP, the authorities are committed to increasing spending for povertyreducing programs and growth enhancing infrastructure investments. They recognize that attaining these objectives requires creation of appropriate fiscal space to preserve fiscal and debt sustainability. To this end, the Government has recently conducted a comprehensive review of the tax policy and tax administration, including tax holidays and mining taxation, with the support of Fund TA. Implementation of the tax system and tax administration 
reforms that would result in a more buoyant, simpler, broadened, equitable and well administered tax regime, will continue. The Implementation of the recommendations of the tax review has started with the 2007 budget, and the authorities will continue renegotiating development agreements with the mining companies. It is expected that these actions will substantially improve tax compliance and sustain revenue performance levels at above 19.0 percent of GDP in the medium-term.

8. In addition to revenue enhancing initiatives, the authorities have made strong progress in developing a medium-term expenditure framework (MTEF) to ensure allocation of higher capital spending in the identified priority sectors. They remain committed to making further progress in improving public expenditure management, with a view to addressing the existing difficulties in budget execution, improving coordination between fiscal and monetary policy, and enhancing absorptive capacity of public investment. To further increase the effectiveness and productivity of the public sector, the authorities will accelerate implementation of the PEMFA reforms, including the IFMIS. They have already taken important steps to strengthen debt management, and will continue enhancing capacity to formulate and execute investment projects.

\section{Monetary and exchange rate policies}

9. The authorities' main objective in monetary policy is to maintain low inflation levels. In this connection, the $\mathrm{BOZ}$ will continue monitoring inflationary pressures and adopt appropriate measures to keep reserve money in line with the targets. The managed floating exchange rate regime has served the economy well, and will continue with BOZ limiting its interventions to correcting transitory overshooting of the exchange rate and meeting its international reserve targets. The authorities are aware of the challenges associated with management of increased foreign currency inflows to monetary policy and competitiveness. Capitalizing on their recent experience and the recommendations of a recent Fund TA, they will use foreign exchange sales to mop up the excess liquidity generated by the increased inflows from the booming mining sector and donor support. To avoid aggravating exchange rate volatility, and raising interest rates, the authorities will carefully plan the time for the sales and continue improving foreign exchange market.

\section{Structural reforms}

10. The authorities' structural reform agenda is mainly aimed at improving conditions for private sector growth, in addition to strengthening public finance management. To this end, they remain committed to enabling a friendly-investment environment and reducing cost of doing business, notably through a combination of deepened implementation of the financial sector development (FSDP) plan, a more open trade regime, and removal of infrastructure bottlenecks especially by developing the energy sector. They are also strongly committed to good governance, and have conducted a participatory process in accordance with the 
Extractive Industries Transparency Initiative (EITI) guidelines and made important progress toward its formal adoption, with the support of the World Bank.

11. Improving access to financial services is important for private sector development and economic growth. In this connection, the authorities are committed to taking steps to strengthen supervision and broaden access to financial services. The BoZ is establishing a framework for risk-based supervision, and is advancing with implementation of the second phase of the FSDP which would help strengthen financial system stability and access to credit. The establishment of a Credit Reference Bureau and an enabling environment for microfinance are also underway and should positively change the credit culture and expand credit and other financial services to rural businesses. The BoZ is also developing the capital market, including support for the creation of an active secondary market in government securities.

12. The authorities are committed to further promoting a more open trade regime. They are playing a pivotal role in regional economic integration within COMESA, and plan to implement the Trade Protocol agreed under SADC. They will streamline procedures and reduce the time required to process exporting and importing goods, with a view to substantially reducing the cost of cross-border trading.

13. Infrastructure bottlenecks have been identified as the main impediment to sustained high growth as they increase the cost of doing business and reduce competitiveness and productivity. In this connection, the authorities intend to upgrade quality, expand capacity and increase efficiency in energy, transport, and telecommunications infrastructure. Particular emphasis would be on increased investments in the energy sector. In doing so, the authorities are aware of the need to improve governance and efficiency of the public utility, ZESCO, including application of cost-based rates to mining companies.

\section{Conclusion}

14. The Zambian authorities' continued commitment to sound macroeconomic policies and structural reforms is translating into robust economic performance over the recent years, contrasting with episodes of stagnation of the past decades. They are conscious that further reform effort is required to consolidate the progress made and sustain high economic growth rates to effectively tackle the still widespread poverty and attain the MDGs. To this end, the authorities count on continued support from the Fund, and are looking forward to the finalization of the discussion on the successor program. They are committed to continue consolidating macroeconomic stability and stepping up implementation of their structural reform agenda through the FNDP, which they consider to be the appropriate framework. 